\title{
Experimental methods for investigating protein adsorption kinetics at surfaces
}

\section{JEREMY J. RAMSDEN}

Department of Biophysical Chemistry, Biocentre of the University, $\mathrm{CH}-+056$ Basle, Switzerland

I. INTRODUCTION 42

I.I Formalism of an adsorbed protein adlayer 44

2. SOLUTION DEPLETION TECHNIQUES 46

2.1 The well-mixed vessel 46

2.2 The packed bed 50

3. GRAVIMETRIC METHODS 50

3.I The quartz crystal microbalance ( $Q C M) \quad 50$

3.2 Surface acoustic wave $(S A W)$ devices 52

4. HYDRODYNAMIC METHODS 53

4. I The diffusivity of colloidal spheres 53

4.2 Capillarity 56

5. ELECTRICAL METHODS 57

5.I Impedance measurements 60

5.2 Electrokinetic methods 66

6. OPTICAL METHODS 67

6.I Ellipsometry 70

6.2 Scanning angle reflectometry $(S A R) \quad 72$

6.3 Integrated optics (IO) 73

6.4 Total internal reflexion spectroscopy (TIRS) 77

6.5 Surface plasmon resonance (SPR) 78

6.6 Evanescent wave light scattering (EWLS) 79

7. LABELLING TECHNIQUES 79

7.I Radiolabelling 80

7.2 Enzyme-linked immunoassay (ELISA) 80

7.3 Total internal reflexion fluorescence microscopy (TIRFM) $\quad 82$

7.4 Resonance energy transfer (RET) 83

8. EVALUATION OF KINETIC PARAMETERS 84

9. COMPARISON OF TECHNIQUES 87

10. THE NATURE OF THE ADSORBED PROTEIN LAYER 9 I

II. ACKNOWLEDGMENT 94 
12. REFERENCES 94

APPENDIX LIST OF PRINCIPAL SYMBOLS IO2

\section{INTRODUCTION}

The adsorption of proteins at the solid-liquid interface is a process of fundamental importance in nature. Extensive reviews (MacRitchie, 1978; Andrade \& Hlady, 1986; Norde, 1986) testify to the strong interest which has been shown in the problem during the past few decades. Norde \& Lyklema (1978) have rightly pointed out that protein adsorption is scientifically intriguing; the phenomenology is complicated and includes many presently apparently irreconcilable observations. The very variety among proteins makes the field complex; it need hardly be said that 'measurements with well-defined model systems carried out under well-controlled conditions' (Norde \& Lyklema, 1978) are an essential precondition to progress in understanding the underlying mechanisms. One of the most valuable approaches to elucidating the mechanism of complicated phenomena is of course to study their kinetics. Yet 'the dynamic aspects of protein adsorption at solid-liquid interfaces have not been studied extensively and only a few papers are available in this area of research' (Sarkar \& Chattoraj, 1992). Since many protein adsorption processes are partly or wholly irreversible, kinetic as opposed to equilibrium measurements assume a particular importance.

One reason for the poverty of results has been the dearth of suitable (reliable, convenient, etc.) experimental techniques. For many decades there was a clear division between techniques such as radioactive tracer labelling which were capable of yielding an accurate figure for the number of protein molecules adsorbed at a surface, but which were too cumbersome to apply to kinetic measurements, and techniques which did allow kinetics to be measured continuously or quasicontinuously, but which involved many untestable assumptions and which even then could often only yield a relative number of adsorbed molecules. Techniques in both categories usually required the adsorbing molecules to be labelled with a radioactive, fluorescent or other type of marker, an often laborious (or expensive) procedure with unknown effects on the transport properties, conformation etc. of the molecule. Over long periods there is also a risk that the labels may become detached.

Theoretical treatment of the problem remained stagnant without the stimulus of usable data. For example, it is only rather recently that it has been realized that the widely used Langmuir adsorption isotherm is not appropriate for the problem (Mura-Galelli et al. I99 I Ramsden, I 993 $b$ ). With the advent of modern, accurate experimental techniques, theoreticians are beginning to realize that the field offers a very rich phenomenology with which the most sophisticated description of adsorption processes can be challenged.

This review will be devoted essentially to the experimental techniques available for characterizing these process. For providing precise, reliable data on the number of molecules at the interface, modern optical methods appear to be the most promising $(\$ 6)$. On the other hand, some of the older methods take on a new significance when used in conjunction with accurate adsorption data provided by 
the optical techniques, for yielding complementary information about the electrostatics of the process, etc. All major techniques which have appeared in the literature are dealt with in this review, although some borderline methods have been omitted. ${ }^{1}$ Emphasis is placed on how the desired results are obtained, i.e. the working equations for each technique, and on what assumptions are needed in order to obtain the desired information.

The areas of research for which a detailed knowledge of protein adsorption is necessary fall into two broad classes. One is the investigation of fundamental biochemical mechanisms of processes such as blood clotting (which involves surface-induced thrombosis, platelet aggregation, etc.) and molecular recognition. Proteins are a special, highly complex, and important case of particles adsorbing at surfaces, a field which is enjoying a strong renaissance among theoreticians (Bartelt \& Privman, 199I), whose current work is concentrated on hard disks, spheres and ellipsoids; it is to be expected that once reliable experimental kinetic results from proteins become available, many new theoretical challenges will be presented. The other class is heuristic: questions to be asked are initially of the type 'how long do I have to leave my microtitre plate exposed to this protein solution in order for a certain surface density of protein to be adsorbed?' The answers will naturally involve, at a deeper level, results from the first class. Examples abound. The kinetics of formation of basement membranes is a rather new field of investigation (Ramsden, 1993a); associated with it is the spreading and proliferation of cells on membranes constituted from various kinds of extracellular matrix molecules (Masuda-Nakagawa \& Wiedemann, 1992), for which it is important to be able to prepare basement membranes in vitro of a definite thickness and packing density. The first step in solid-phase immunoassays (Porstmann \& Kiessig, 1992) is the adsorption of antigens or antibodies to a solid surface; although hitherto this step has not usually been carried out very quantitatively, the future development of such assays will certainly demand it. The subsequent binding of analyte to the capture layer can be quantitatively investigated by many of the techniques to be discussed here. The deposition of proteins on electron microscope grids, and the screening of materials for affinity chromatography (Kopaciewicz, 1983; Place et al. I 99 I), are further fields in which a quantitative knowledge of the kinetics of protein adsorption is indispensible for obtaining deeper insight; others are biocompatibility in medicine, which, with the growing use of artificial implants, internal organs and in vivo sensors is growing in importance, and the fouling of filters used in ultrafiltration and other techniques of protein purification (van den Oetelaar et al. 1989).

The criteria for the methods of choice are: the desired information (number of molecules per unit area of surface) to be obtained as directly as possible from the measurements; the measurements to be on-line, i.e. continuous or quasicontinuous (to permit accurate kinetics to be obtained); the surface and other conditions to be well-defined; no labelling or other modifications of the proteins to be necessary. Only a few of the available methods will be seen to meet these

\footnotetext{
1 Of course, it will always be possible to devise new techniques. As an example of an exotic method, the quantum yield of latent image formation in silver balide crystallites could be measured, for it is dependent on layers of protein such as gelatin at the crystalfithinjuacBicl. I
} 


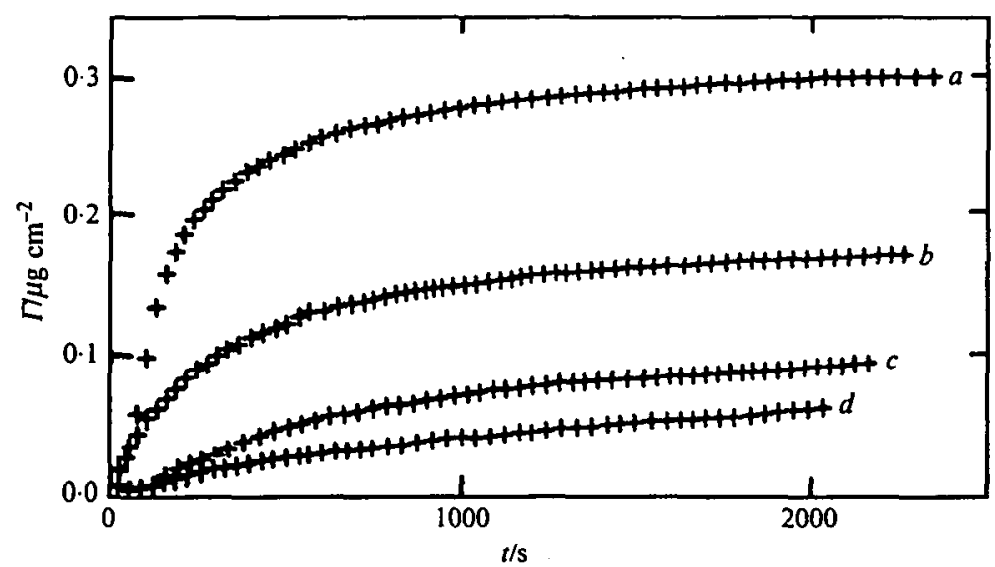

Fig. I. Adsorption of apotransferrin on a $\mathrm{SiTiO}_{2}$ surface, which was a planar optical waveguide. Measurement of the velocities of two guided modes allowed the calculation of $\Gamma$ (See $\$ \$ 6,6.3$ for discussion). The protein was dissolved at a concentration of $100 \mu \mathrm{g} / \mathrm{cm}^{3}$ in tris- $\mathrm{HCl}$ buffer, $\mathrm{pH} 8.0$, at concentrations of $2 \mathrm{mM}$ (curve $a$ ) and $10 \mathrm{~mm}$ (curves $b-d$ ), with $\mathrm{NaCl}$ added (curves $c, 0.1 \mathrm{M}$; curve $d, 0.5 \mathrm{M}$ ). The protein solution flowed across the waveguide surface at a mean velocity of $0.14 \mathrm{~mm} / \mathrm{s}$. Full details are given in Ramsden \& Prenosil (1993).

criteria satisfactorily, but others may be useful for providing complementary information on specific aspects of the adsorption process. As mentioned above, the emphasis will be on the working equations and how the desired information is obtained from the measurements. There will be practically no discussion on specific instrumentation, which is extensively documented in the literature. Techniques dealing exclusively with the air-water interface are excluded.

Figure I shows an example of data obtained using an integrated optics technique $(\$ 6.3)$. The protein adsorption is taking place under conditions of differing ionic strength. The figure demonstrates that extraordinarily small and subtle changes in deposition behaviour can readily be detected.

Further examples of adsorption kinetics will be given during the discussion of individual methods. Practically no truly comparative measurements using two or more techniques have been carried out on the same system (i.e. identical protein, surface, solution conditions, etc.); given the variety of protein behaviour encountered, the few comparisons which have been reported scarcely suffice as a basis from which to generalize. One does not necessarily expect the same numbers to come out from measurements carried out using different methods. The surface excess $\Gamma$ of adsorbed proteins is, in fact, only the first stage of knowledge. It is one of the objects of this review to show that different techniques yield insight into different aspects of the problem, such as the conformation and orientation of proteins in monolayers, and the packing density of proteins in multilayers.

\section{I.I Formalism of an adsorbed protein adlayer}

Before embarking upon a discussion of individual techniques, we shall establish the basic terminology and definitions to be used throughout. Fig. $2 a$ shows the 


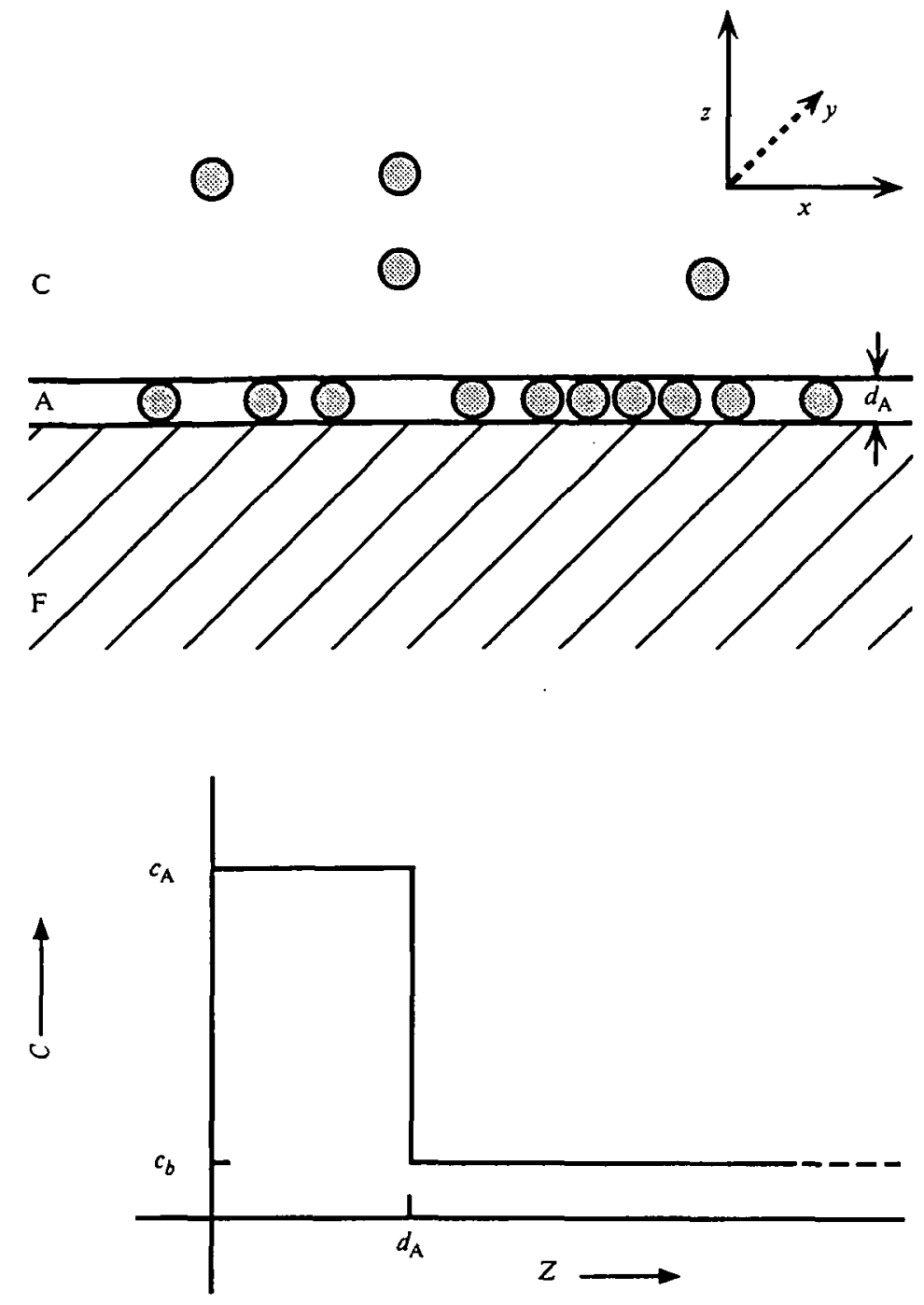

Fig. 2. (a) Schematic drawing of a formalized interface, showing the cover layer $C$, containing the freely suspended protein molecules (shaded circles) at a volume concentration $c_{b}$, the adlayer $\mathrm{A}$ of adsorbed protein molecules, characterized by a surface excess $\Gamma$, and the solid substrate $F$ (Festkörper) at the surface of which adsorption takes place. Inset:

coordinate system used throughout this review. The adsorbing surface lies in the $x, y$ plane.

(b) Concentration profile of the idealized adlayer.

cross section of an idealized interface. The most intuitively direct way of observing it is by means of microscopy. Light microscopes do not have adequate resolving power for proteins; electron microscopy is not easily applicable to kinetic measurements. ${ }^{2}$ Its power lies in the possibility of direct investigation of the conformation of individual molecules and their lateral distribution (Nygren \&

: It is possible to prepare a sequence of grids, each grid having been exposed to a protein solution for a different length of time (Nygren \& Stenberg, $1990 a$ ). Since a separate substrate must be used for each exposure, and the preparation of identical substrates is one of the most difficult problems in the investigation of adsorption problems, this method cannot be used for obtaining very precise kinetic curves. 
Stenberg, I $990 a, b ;$ Nygren, I 992 ; Nygren \& Karlsson, 1992). In this respect it is truly complementary to the kinetic methods described in later sections, in which an area of the surface containing hundreds or thousands of molecules is integrated; in these methods, lateral interaction must be inferred from the kinetics, if they are precise enough!

For many purposes, it is precisely knowledge of the average properties which is sought. The primary quantity of interest is $\Gamma$, the mean mass or number of molecules per unit area, which is related to the fractional surface coverage $\theta$ according to

$\Gamma=\theta m / a$,

where $m$ is the mass per molecule (when $\Gamma$ denotes the number of molecules per unit area, $m=\mathrm{I}$ ) and $a$ the area per molecule. Some of the methods to be discussed yield primarily $\Gamma$, whereas others give $\theta$ directly; provided the ratio $a / m$ is known, one may always be converted into the other. Fig. $2 b$ shows the concentration profile of the idealized interface. According to the Gibbs convention,

$\Gamma=\int_{0}^{\infty}\left(c(z)-c_{b}\right) \mathrm{d} z$

where $c$ denotes protein concentration (subscript $b=$ bulk); for the idealized profile shown in Fig. $2 b$, which is applicable to protein monolayers, this becomes:

$\Gamma=\left(c_{\mathrm{A}}-c_{b}\right) d_{\mathrm{A}}$,

and for $c_{\mathrm{A}} \gg c_{b}$, we have simply

$\Gamma=c_{\mathrm{A}} d_{\mathrm{A}}$.

\section{SOLUTION DEPLETION TECHNIQUES}

\section{I The well-mixed vessel}

This is an extremely simple method. The material on which adsorption is to take place is put, in highly comminuted form in order to maximize its surface area $A$, in a vessel equipped with some means of agitating its contents. A known amount of dissolved protein (concentration $c_{0}$ ) is added, and immediately well stirred (mixing is assumed to be instantaneous). After a desired interval $t$, the particles are separated from the supernatant-usually by gravity, which may call for centrifugation if the particles are small - and the concentration of protein $c_{b}$ in the bulk supernatant measured. Clearly

$\Gamma=\left(c_{0}-c_{b}\right) V / A$,

where $V$ is the total volume of liquid in the vessel. The method has the advantages of almost unlimited flexibility regarding the nature of the solid adsorbent, and of the way used to determine the concentration of protein in the supernatant (ultraviolet-visible, circular dichroism, photoluminescence spectroscopies, etc.). On the other hand it possesses the obvious disadvantage of being intermittent, and is therefore only useful for slow kinetics, for the adsorption process must be 


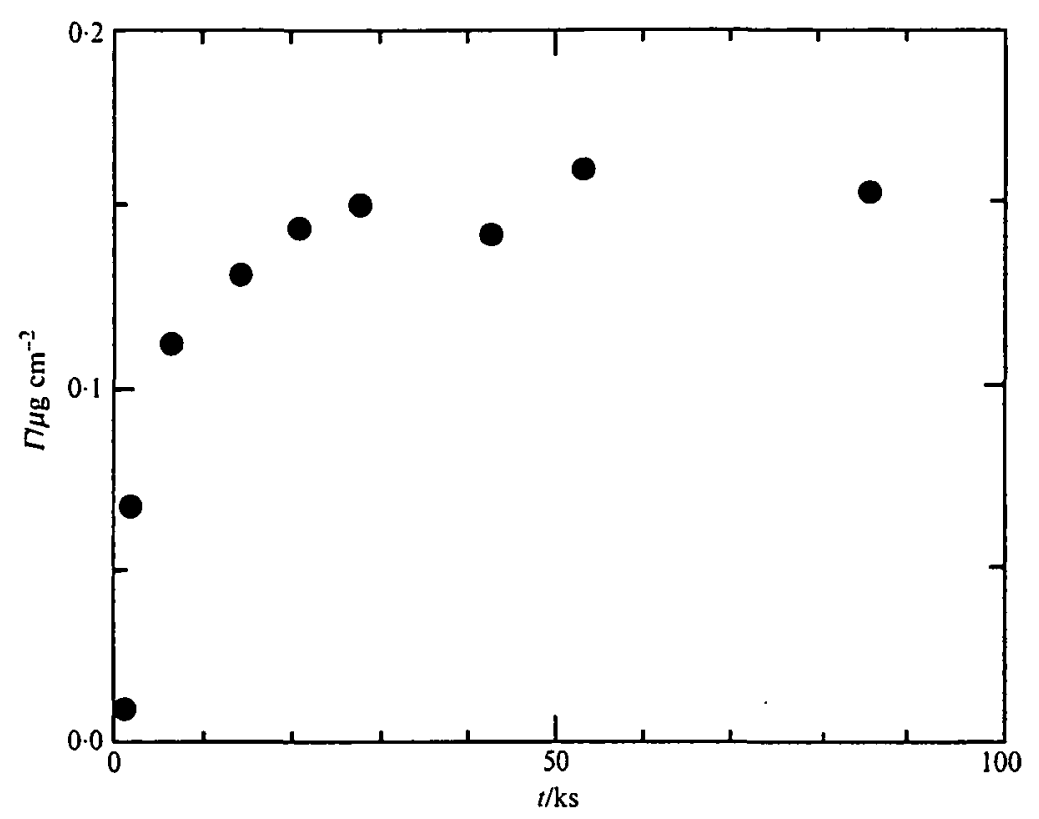

Fig. 3. The adsorption of human serum albumin to hydroxyapatite powder (specific surface $45 \mathrm{~m}^{2} / \mathrm{g}$ ) at $27^{\circ} \mathrm{C}$ measured using a solution depletion technique. The protein was labelled with ${ }^{125} I$ to make detection more sensitive. Initially, sufficient protein solution was added to $50 \mathrm{mg}$ powder in $13.3 \mathrm{~cm}^{3}$ buffer ( $50 \mathrm{~mm}$ tris- $\mathrm{HCl}, \mathrm{pH} 7.35$, plus $0.15 \mathrm{M}-\mathrm{NaCl}$ ) to ensure that the final dissolved protein concentration lay in the range $150-300 \mu \mathrm{g} / \mathrm{cm}^{3}$ (Mura-Galelli et al. ( $99 \mathrm{r})$.

interrupted in order to measure $c_{b}$. Fig. 3 shows an example of kinetics obtained with the technique. Sometimes, if the adsorption kinetics are being determined in conjunction with some other property of the protein, e.g. enzymatic activity, which must also be determined intermittently, this is not a great disadvantage (e.g. Veselova et al. 1987). More severe problems are: the often ill-defined nature of the surface, for the comminution process-crushing, milling or grinding-may introduce numerous defects into the surface ${ }^{3}$ the difficulty of precisely measuring the area $A$ available for adsorption; and the difficulty of ensuring that mixing really is close to instantaneous. The solutions of these problems impose conflicting requirements. The smaller the particles, the larger is $A$ and hence the difference $c_{0}-c_{b}$ for a given mass of adsorbing substance, and hence the precision of the determination of $\Gamma$, but the less the particles will resemble 'bulk surface' (Sinanoglu, I98I), and the harder it is to separate the particles from the supernatant (centrifugation at high $g$ may disturb the surface-solution

3 The particles may also be produced by nucleation and growth, which can result in well-defined, homogeneous populations of particles with the same crystal structure as that of bulk material (Ramsden, 1985,1987 ), but the growth procedure has to be determined separately for each material. Inevitably high proportion of the surface atoms of the crystallites are at edge or corner sites, for which the adsorption energies are usually different from those of atoms in an infinite plane. Latices can be prepared as small smooth spheres, but then the adsorbing surface is no longer planar, but highly curved, which may change the energy of adsorption, since the surface tension of the solid is curvature-dependent (Vogelsberger $\&$ Marx, 1976); the radius of curvature of the solid phase may even be comparable to the linear dimensions of the adsorbed molecules and hence affect the adsorption process directly. 


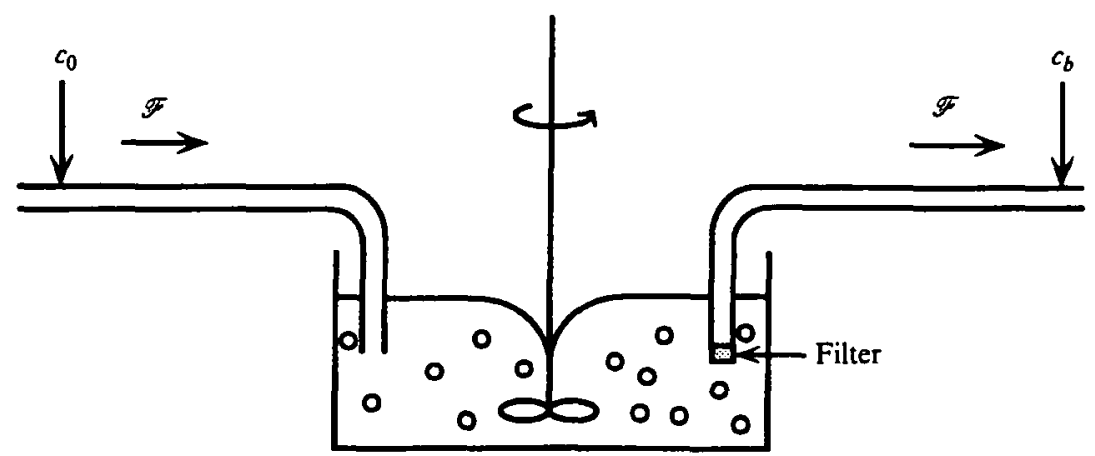

Fig. 4. The continuous solution depletion technique (CSD). A protein solution at a concentration $c_{0}$ flows with rate $\mathscr{F}$ into the vessel of volume $V$ containing particles with a total surface area $A$. Mixing is assumed to be instantaneous. Fluid is pumped out with the same rate $\mathscr{F}$, and the outflowing concentration $c_{b}$ (i.e. the bulk solution concentration in the vessel) monitored continuously downstream.

equilibrium). The hydrodynamics is extremely ill-defined; at the moment of adding the protein solution some particles will be in the presence of a very high protein concentration, while others will only ever see a highly depleted solution. The method is not useful for detailed mechanistic investigations; it can straightforwardly answer the question 'how much protein has adsorbed on one gram of this powder?' when the powder is to be used for another part of the experiment.

Some of the difficulties are overcome by converting the technique into a continuous one. In the method of flow dialysis (Colowick \& Womack, 1969, Laane et al. 198I), one wall of the vessel in which adsorption is taking place ('upper chamber') is made into a semipermeable membrane, through which free molecules, but not the particles to which they can and do adsorb, can diffuse. The same solvent as in the upper chamber is continuously pumped over the other side of the membrane ('lower chamber'). Free molecules diffuse across the membrane at rates proportional to their concentration in the upper chamber; their concentrations in the flowing liquid can be determined downstream. Mass balance gives the following equation:

$\mathrm{d} \nu / \mathrm{d} t=c_{b} K_{c}-\nu \mathscr{F} / B$,

where $K_{c}$ is a cell constant depending on the permeability of the membrane and $\nu$ is the number of free molecules in the lower chamber, whose volume is $B . K_{c}$ can be determined in a control experiment in which adsorbent particles are absent from the upper chamber. Under steady-state conditions $\mathrm{d} \nu / \mathrm{d} t \approx 0$, and measurement of the downstream concentration $c_{2}$ affords $\nu\left(=c_{2} B\right)$ and hence $c_{b}$. Problems arise with kinetic determinations if the time taken to reach steady state is long, i.e. comparable to the characteristic duration of the adsorption reaction. If this is not the case $\Gamma$ can be determined from $c_{b}$ using equation (5). Steady state can be achieved quickly by making the volume $B$ of the lower chamber very small (Feldman, 1978). 


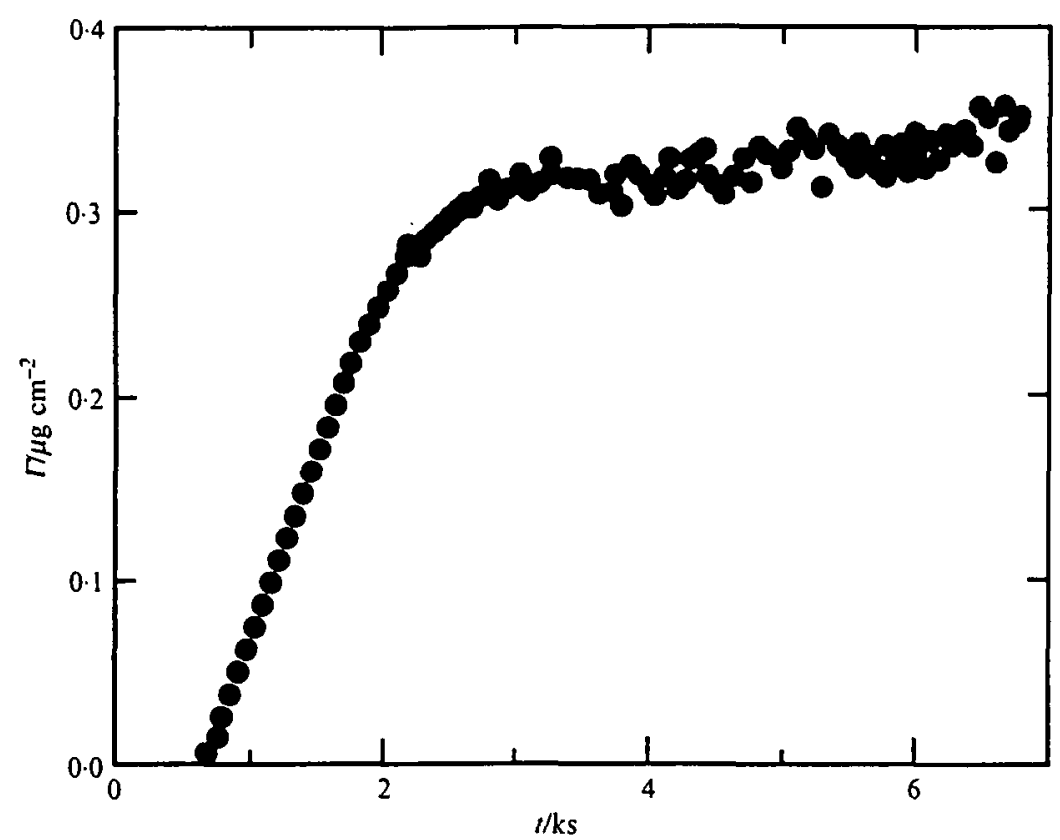

Fig. 5. Application of the CSD technique. The protein is fibrinogen, of which $c a .4 \%$ has been labelled with ${ }^{125} \mathrm{I}$ to aid detection, dissolved at a concentration $c_{0}$ of $95 \mu \mathrm{g} / \mathrm{cm}^{3}$. The vessel has a volume $V=14.6 \mathrm{~cm}^{3}$, flow rate $\mathscr{F}=2.5 \mathrm{~mm}^{3} / \mathrm{s}$. The substrate is soda lime glass beads, diameter $4 \mathrm{I} \pm \mathrm{I} \mu \mathrm{m}$, with a total area of $1030 \mathrm{~cm}^{3}$. The curious shift of the start of adsorption along the time axis is due to a dead volume of ca. $1{ }^{1} 5 \mathrm{~cm}^{3}$ (Cornelius et al. 1992).

Another way (Fig. 4) of effectively sampling the supernatant concentration is to pump protein solution continuously with rate $\mathscr{F}$ into the vessel containing the adsorbing particles, and pump supernatant out at the same rate, a suitable filter preventing the removal of the solid particles (Aptel et al. 1988; Cornelius et al. 1992). Mass balance with respect to protein gives the following equation:

$\mathscr{F} c_{0}=\mathscr{F} c_{b}+V \mathrm{~d} c_{b} / \mathrm{d} t+A \mathrm{~d} \Gamma / \mathrm{d} t$.

This equation can be solved provided another equation linking $\Gamma$ with $c_{b}$ can be found. Aptel et al. ( 1988 ) used the Langmuir isotherm and solved the resulting second-order nonlinear differential equation numerically. Clearly this procedure is unsuitable if the form of the adsorption isotherm is not known a priori; Aptel et al. circumvented the problem by dividing the measured kinetics into several sections, each of which were assumed individually to follow the Langmuir isotherm, with their own distinct parameters. Cornelius et al. (1992) integrated each term in equation (7) with respect to time; rearranging, one obtains

$\Gamma=\left[\mathscr{F}\left(c_{0}-\int_{0}^{t} c_{b} \mathrm{~d} t\right)-V c_{b}\right] A^{-1}$

Fig. 5 shows data obtained using the method with equation $(7 a)$. Clearly, an excellent time resolution is obtainable. 


\subsection{The packed bed}

This technique, in which small particles are packed into a column through which the solution containing the protein is forced, has received a great deal of attention because of its industrial importance in separation and other processes. The protein concentration in solution is not constant but decreases towards the downstream end of the column as protein adsorbs on the particles, and in practice the particles are porous. Both these factors complicate the analysis, in which mass balance is considered in an element of the packed bed (Lee \& Weber, 1969), or in an element of the moving liquid (Leaver et al. 1992), and the measured breakthrough curves simulated. They depend upon various parameters, including adsorption and desorption rate constants characteristic of the material out of which the packed bed is made and the protein, prevailing salt concentration, $\mathrm{pH}$, etc. The simulations allow parameters determined from well-defined, planar systems to be used to predict operating parameters of packed bed separators. The inverse problem, to determine the intrinsic adsorption parameters from the breakthrough curves, is a complicated numerical problem which does not seem to have been attempted.

\section{GRAVIMETRIC METHODS}

Twenty of the 2 I classes of crystals which lack a centre of symmetry are piezoelectric; that is, the crystals become polarized under the influence of external stresses (Curie \& Curie, 1880). Conversely, an electric field applied to a piezoelectric crystal induces a stress. The application of an alternating electric field produces an alternating stress resulting in acoustic standing waves in the crystal (Cady, I 946). These vibrations occur at a characteristic resonant frequency $f$. Fig. $6 a$ shows their equivalent circuit. The figure of merit $Q$ of such crystals can be as high as $10^{4}-10^{5}$, and they are widely used in frequency control and other electronic applications. As in the case of the familiar clock pendulum, the resonant frequency is inversely proportional to the square root of the oscillating mass. This principle is used in a family of gravimetric methods, in which adsorption is allowed to occur on the surface of a vibrating quartz crystal (Ward \& Buttry, 1990; Schumacher, I990), thus diminishing its resonant frequency. Various modes of vibration are possible, of which two, dealt with below, have found application in gravimetric sensors.

\section{I The quartz crystal microbalance $(Q C M)$}

The electric field is applied between two metallic electrodes (of area $A$ ) evaporated onto the surface of the crystal (Fig. $6 b$ ). The vibration mode is thickness shear, and the resonant frequency given by (Cady, r 946; Sauerbrey, 1959):

$f=\frac{m}{2 d_{F}} \sqrt{\frac{\mu_{F}}{\rho_{F}}}$ 


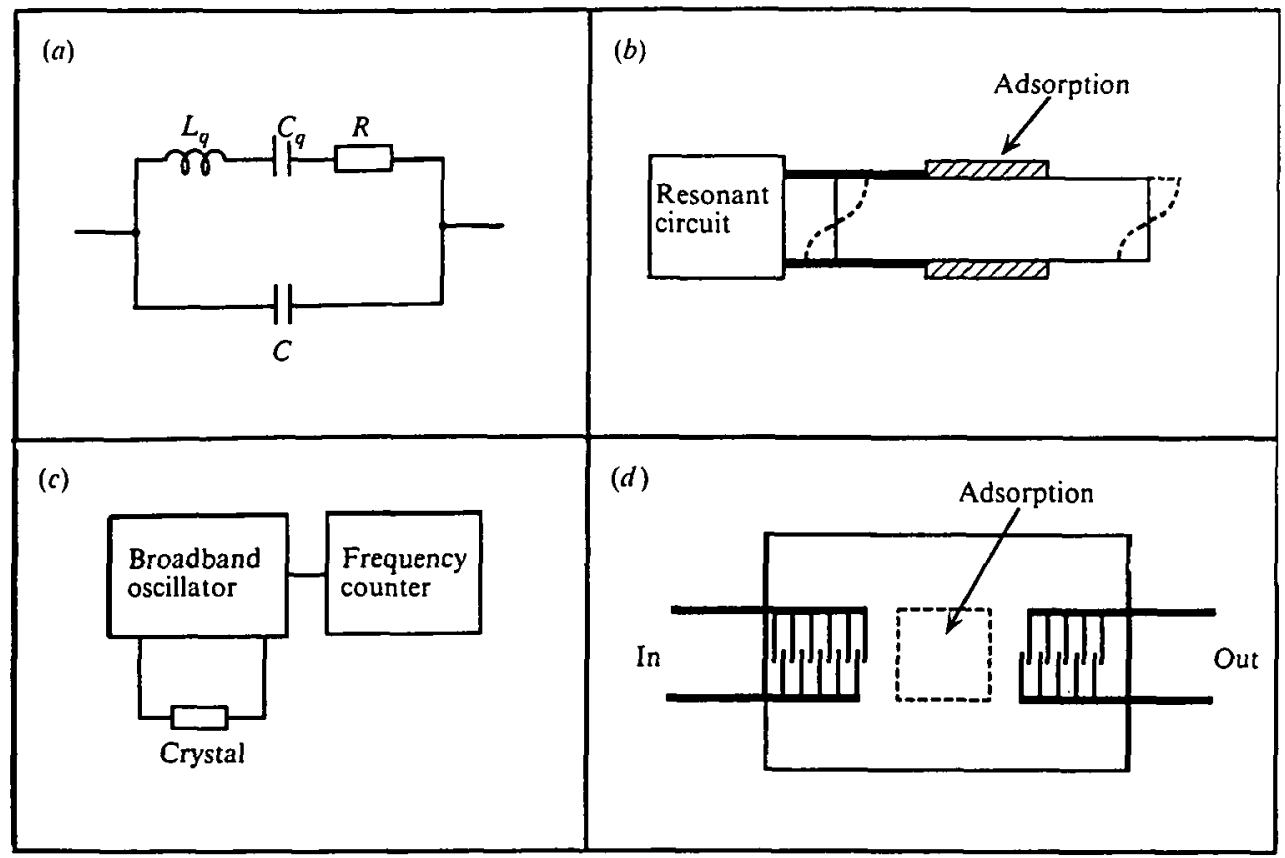

Fig. 6. (a) Equivalent circuit of the quartz crystal resonator (Cady, 1946). The inductance $L_{q}$ represents the inertial mass of the crystal and the reciprocal capacitance $1 / C_{q}$ its shear modulus. $C$ is the electrical capacitance of the crystal; typically $C_{q} / C \approx 10^{-3}$. The resonant frequency of the crystal is $I /\left(2 \pi \sqrt[V]{ }\left(L_{q} C_{q}\right)\right)$. The resistance $R$ represents various losses; the figure of merit $Q$ is $\sqrt{ }\left(L_{q} / C_{q}\right) / R$. (b) Side view of the quartz crystal microbalance undergoing thickness shear vibrations. The thickened lines represent metal electrodes evaporated onto the crystal surface. (c) General scheme for measuring the resonant frequency of QCM or SAW devices. (d) Plan of a surface acoustic wave device. The heavy lines represent the interdigitated metal electrodes which are normally used to excite and receive the waves.

where $m$ is the mode number of the vibration, $\mu_{F}$ the shear modulus of the crystal, $\rho_{\mathrm{F}}$ its density and $d_{\mathrm{F}}$ its thickness. For the commonly used AT-cut quartz of millimetre or submillimetre thickness, $f$ is typically a few $\mathrm{MHz}$, and can readily be determined to an extremely high accuracy by making the crystal part of a resonant circuit (Sauerbrey, 1959). If proteins are deposited at the antinode of the standing wave propagating across the thickness of the crystal, a frequency change $\Delta f$ occurs as if the thickness of the crystal increased by an amount $d_{A}=\Gamma / \rho_{\mathrm{A}} \equiv=\Delta d_{F}=\Gamma / \rho_{F}$ (Sauerbrey, I 959), i.e.

$\frac{\Delta f}{f}=-\frac{d_{\mathrm{A}}}{d_{\mathrm{F}}}=-\frac{\Gamma}{\rho_{\mathrm{F}} d_{\mathrm{F}}}$

This equation is exact for infinitely thin films with the same acoustic impedance as that of quartz. An accuracy of $\Delta f / f$ of $\sim 1_{1} 0^{-7}$ corresponds to a mass resolution of a few $\mathrm{ng} / \mathrm{cm}^{2}$ for a plate $0 . \mathrm{mm}$ thick, and would take $\mathrm{Ios}$ to determine. It is important to remember that equations $(8-9)$ represent a limiting case (perfectly. 
elastic and nonviscous materials) of a more general equation which would include the viscosity and shear modulus of the adsorbed protein layer, but in general these attributes of the adlayer are not known. They may be inferred from the complete impedance spectrum of the device.

For use in protein adsorption experiments, the crystal must be immersed in an (aqueous) protein solution. The QCM thereby loses some of its simplicity, for the elastic shear waves in the crystal are now coupled to viscous shear waves in the liquid, which die away exponentially with a characteristic length $\delta=$ $\sqrt{ }\left[2 \eta_{\mathrm{C}} /\left(2 \pi f \rho_{\mathrm{C}}\right)\right]$ (typically $\sim 250 \mathrm{~nm}$ for aqueous solutions). In fact, the vibrating quartz crystal was first used to specifically study the elastic properties of liquids (Mason et al. 1949). The presence of the liquid causes a shift $\delta f$ in the resonant frequency of a crystal; if one side of the crystal is in contact with a liquid of absolute viscosity $\eta_{\mathrm{C}}$ and density $\rho_{\mathrm{C}}$, then by matching the shear waves in the two media at their interface (Kanazawa $\&$ Gordon, 1985):

$\delta f=-f_{0}^{\frac{3}{3}} \sqrt{ }\left[\rho_{\mathrm{C}} \eta_{\mathrm{C}} /\left(\pi \rho_{\mathrm{F}} \mu_{\mathrm{F}}\right)\right]$,

where $f_{0}$ is the frequency in the absence of liquid. This expression assumes that $\eta_{\mathrm{C}}$ is independent of frequency, which is not necessarily the case (Mason et al. 1949). The mass sensitivity of the crystal immersed in liquid is then the same as in air, i.e. equation (9) may be used to determine $\Gamma$ from $\Delta f$. The figure of merit $Q$ in the presence of liquid may only be $\sim \mathrm{r}^{3}$, however; hence the determination of the resonant frequency may no longer be so precise. If the vibrating surface is rough, liquid confined in surface cavities will be dragged along with the crystal (Schumacher, 1990), behaving as an adsorbed adlayer of a thickness equal to the characteristic radius of the rugosity. Where the adlayer under investigation consists of randomly adsorbed protein molecules, the surface is necessarily rugose with a characteristic radius $d_{\mathrm{A}} / 2$, and this could lead to large errors, of order $\Gamma$, in the determination of $\Gamma$.

\subsection{Surface acoustic wave (SAW) devices}

In contrast to thickness shear vibration, Rayleigh surface acoustic waves are transmitted parallel to the solution-surface interface across a region of the crystal on which deposition is taking place (White, I970; Roederer \& Bastiaans, 1983). Typical frequencies are in the same range as for the QCM, i.e. $\sim$ Io $\mathrm{MHz}$, with wavelengths $\lambda \sim 300 \mu \mathrm{m}$. Transmitter and receiver electrodes-typically identical, interdigitated, structures - are deposited on the same face of the crystal (Fig. $6 d$ ). The Sauerbrey equations (8-9) can again be used to determine $\Gamma$ from $\Delta f$. SAW devices have been successfully applied to applications involving contact with a liquid (Calabrese et al. 1987), albeit with the same reservations as with the $\mathrm{QCM}$ regarding the variation of $\eta_{\mathrm{C}}$ with frequency. If the substrate is thick (i.e. $d_{\mathrm{F}}>5 \lambda$ ), attenuation of the Rayleigh (surface) modes makes the device insensitive. For $d_{\mathrm{F}}<{ }_{5} \lambda$, the devices have been shown to work successfully, although the dominant mode of energy propagation is not quite clear (Calabrese et al. 1987 ; see table $\mathrm{I}$ of that paper). 


\section{HYDRODYNAMIC METHODS}

These techniques rely on the friction engendered between solid and liquid phases in relative motion to each other. After an initial period of acceleration under a constant applied force, a steady state is reached and the characteristic velocity of particles or liquid is observed. The accumulation of proteins at the solid-liquid interface increases the frictional coefficient, and from this increase the amount of adsorbed material can be calculated.

\section{I The diffusivity of colloidal spheres}

The adsorbent is a collection of monodisperse spheres of known size and structure. According to the Stokes-Einstein relation,

$D=k T /\left(6 \pi s \eta_{\mathrm{C}}\right)$,

where $D$ is the translational diffusion coefficient, $s$ the radius of the sphere, and $\eta_{\mathrm{C}}$ the absolute viscosity of the solution. A layer of adsorbed proteins causes the diameter to increase by $d_{A}$ (Fig. 7a), hence the diffusivity decreases by $\Delta D$ :

$\Delta D=\frac{k T}{6 \pi \eta_{\mathrm{c}}}\left(\frac{\mathrm{I}}{s}-\frac{\mathrm{I}}{s+d_{\mathrm{A}}}\right)$

from which $d_{\mathrm{A}}$ may be obtained. This idea has been used by Duckworth et al. (1978) to determine the thickness of adsorbed polymer layers on small latex spheres. There are three main problems: (i) since it is a difference method it is liable to inaccuracies; (ii) as will be discussed below, equation ( 12 ) fails to take into account hydrodynamic interactions between adsorbed proteins; (iii) without knowing the density of protein packing, there is no definite connexion between the measured $d_{A}$ and $\Gamma$.

Bloomfield et al. (1967) and McCammon et al. (1975) have obtained numerical and analytical results respectively for the translation diffusional coefficient $D$ of a sphere to which discrete particles ('subunits') are attached. The simple equation ( 1 I ) can still be used provided that the radius $s$ is replaced by the equivalent hydrodynamic radius of the assembly $s^{\prime}$, given by:

$s^{\prime}=\left(s+d_{\mathrm{A}}\right)[\mathrm{I}-\mathrm{I} /(\gamma \tanh 2 \gamma)]$,

where

$\gamma=1 \cdot 35 \sqrt{ } \theta$.

These equations are valid for $d_{\mathrm{A}} \ll s$ (McCammon et al. 1975). By substituting $s^{\prime}$ for $s$ in ( $\mathrm{II}$ ) it can be seen that above $\theta \approx 0.5, D$ does not change very much with $\theta$, as had already been found by Bloomfield et al. (1967). Therefore hydrodynamic measurements are not very sensitive at high $\theta .^{4}$

Equations (13) and (14) show that the measured $D$ or $\Delta D$ is a function of two parameters characterizing the adlayer, $d_{\mathrm{A}}$ and $\theta$, and hence without an

$\checkmark$ The mean value of $\theta$ for randomly sequentially adsorbed particles (see $\S 8$ ) cannot in any case exceed 0.54. 


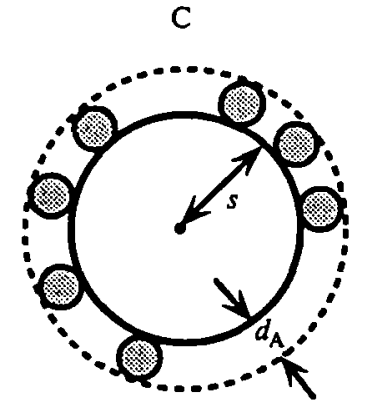

(a)

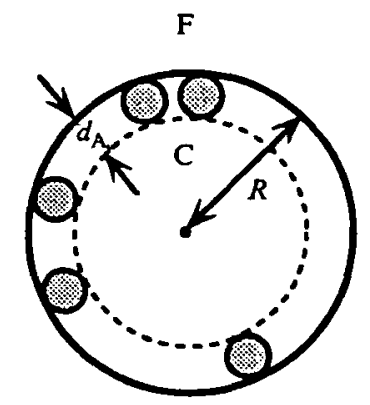

(b)

Fig. 7. (a) Spherical colloidal particle (radius s) undergoing diffusive or directed motion in medium $\mathrm{C}$. Shaded circles represent adsorbed protein molecules forming an adlayer of thickness $d_{\mathrm{A}^{\prime}}$ (b) A capillary of radius $R$ bored through medium $\mathrm{F}$ and filled with medium C. Protein molecules adsorbed on the inner walls diminish the capillary radius by adlayer thickness $d_{A}$.

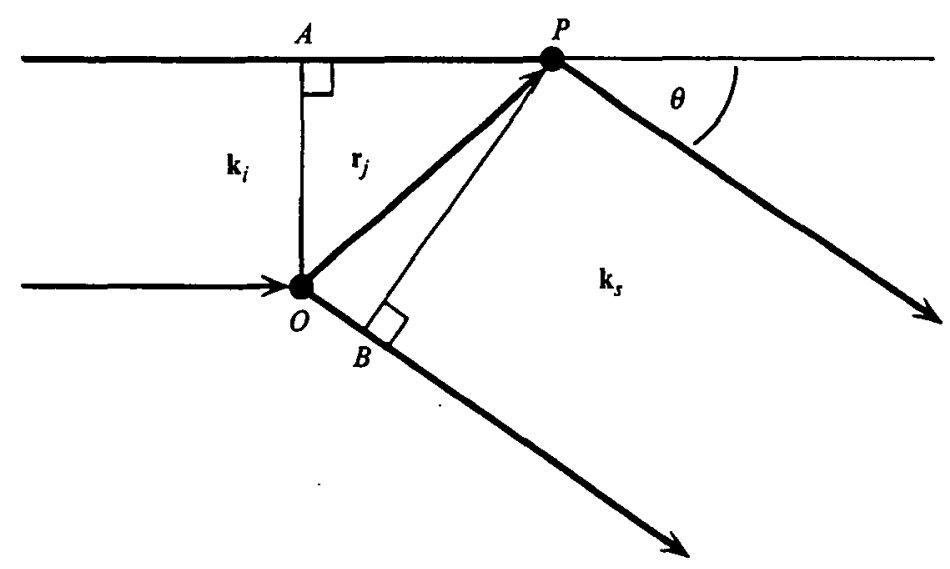

Fig. 8. Light (wave vector $\mathbf{k}_{i}$ ) incident upon the scattering volume is scattered; the scattered light (wave vector $\mathbf{k}_{\mathrm{s}}$ ) is measured at an angle $\theta$ from the incident beam. An arbitrarily chosen reference particle is situated at $O$, and a second particle at $P$.

independent determination of $d_{\mathrm{A}}$ (which may, however, be available from a knowledge of the protein structure), $\theta$ cannot be uniquely deduced from the diffusivity of colloidal spheres.

A convenient way of determining $D$ is through quasi-elastic light scattering (QELS), otherwise known as light-beating spectroscopy, optical mixing spectroscopy, photon correlation spectroscopy, etc. (Cummins \& Swinney, I 970; Cummins \& Pike, 1974; Chu, 1974; Berne \& Pecora, 1975). In this method, a small volume of a suspension of identical (and spherically symmetrical) colloidal particles containing $\nu$ particles is illuminated by a collimated beam of coherent (laser) light (with wave vector $\mathbf{k}_{i}$ ), and the intensity of scattered light (with wave vector $\mathbf{k}_{s}$ ) measured at a certain angle $\theta$ (Fig. 8). By way of illustration, let us take just one pair of particles whose relative position is given by the vector $\mathbf{r}_{j}$. Defining the scattering vector $\mathbf{K}$ as $\mathbf{k}_{i}-\mathbf{k}_{i}$, and hence $K=|\mathbf{K}|=(4 \pi / \lambda) \sin (\theta / 2)$, the phase 
angle of light scattered from the pair of particles is $\mathbf{K} \cdot \mathbf{r}_{j}$. Considering now the assembly of $\nu$ randomly placed particles in the scattering volume, the total scattered field is simply the sum of the fields scattered by the individual particles, namely

$E_{s}=\sum_{j=1}^{v} A \exp \left(-i \mathbf{K} \cdot \mathbf{r}_{j}\right)$

where $A$ is the amplitude scattered by a single particle. The average total intensity $I_{s}=\left\langle\left|E_{s}\right|^{2}\right\rangle=\nu\left\langle|A|^{2}\right\rangle$, angular brackets denoting the average over time. Since the particles are in constant motion (Brown, 1828 ), the $\mathbf{r}_{j}$ and hence $E_{s}$ are time dependent; the measured signal cannot change infinitely quickly, however, but with a characteristic correlation or coherence time $\tau_{c}$, whose magnitude is determined by the motion of the particles and hence by $D$. Let us measure ${ }^{5} E_{s}$ beginning at some arbitrary epoch $t$ and continuing for an interval $\mathscr{T}$, and repeat the measurements over $m=1,2, \ldots, \mathscr{M}$ successive consecutive discrete intervals all of duration $\mathscr{T}$. The normalized discrete autocorrelation function $\mathscr{C}$ of the random signal $E_{s}$ is

$\mathscr{C}(\tau)=\left\langle E_{s}(t) E_{s}^{*}(t+\tau) /\left(E_{s}(t) E_{s}^{*}(t)\right)\right\rangle$,

where $\tau$ is the interval between the epoch $t$ and the $m$ th discrete measuring interval. ${ }^{6} \mathscr{C}(\tau)$ equals the product of the phase and amplitude auto-correlation functions. Since the mean square value of $E_{s}$ does not change with time, the amplitude autocorrelation function simply equals $A^{2}$, and we obtain for the phase autocorrelation function:

$\mathscr{C}(\tau)=\frac{1}{\nu} \sum_{j=1}^{\nu}\left\langle\exp \left(i \mathbf{K} \cdot\left[\mathbf{r}_{j}(t+\tau)-\mathbf{r}_{j}(t)\right]\right)\right\rangle$.

For scatterers undergoing translational diffusion (Brownian motion) characterized by their diffusivity $D$, carrying out ensemble average and sum (Cummins \& Swinney, I 970) yields:

$\mathscr{C}(\tau)=\exp \left(-D K^{2} \tau\right)$

A plot of the logarithm of the autocorrelation function against $\tau$ therefore yields a straight line of slope $D K^{2}$, which is the reciprocal of the coherence time $\tau_{c}$. For measuring $D$ of small colloidal particles a million channels of duration $0.1 \mathrm{~ms}$ would be typical measurement parameters.

Uzgiris \& Fromageot (1976) have pointed out that the thickness $d_{A}$ which is required in order to be able to determine $\theta$ or $\Gamma$ from $D$ (equations I I-I4) can be obtained from the way the scattered light intensity $I_{\delta}$ changes as a film is adsorbed onto the particle surface. Provided that $s \ll \lambda$, the polarizability $\alpha_{\mathrm{F}}$ of the naked sphere is, according to the Rayleigh approximation,

$\alpha_{\mathrm{F}}=s^{3}\left(n_{\mathrm{F}}^{2}-n_{\mathrm{C}}^{2}\right) /\left(n_{\mathrm{F}}^{2}+2 n_{\mathrm{C}}^{2}\right)$.

3 For details of how the measurements are carried out, namely, how the photons in the scattered field are converted to a train of photoelectrons via a photomultiplier tube, see Cummins \& Swinney (1970).

6 One may also measure the optical power spectrum, which is just the Fourier transform of the desired autocorrelation function. 
If $\Delta I_{s}$ is the change in scattered intensity due to the adsorption of an adlayer, then (Uzgiris \& Fromageot, 1976)

$\frac{\Delta I_{s}}{I_{s}}=\frac{\alpha_{\mathrm{FA}}^{2}-\alpha_{\mathrm{F}}^{2}}{\alpha_{\mathrm{F}}^{2}}=\frac{6\left(n_{\mathrm{A}}^{2}-n_{\mathrm{C}}^{2}\right)\left(n_{\mathrm{S}}^{2}+2 n_{\mathrm{A}}^{2}\right)}{3 n_{\mathrm{A}}^{2}\left(n_{\mathrm{S}}^{2}-n_{\mathrm{C}}^{2}\right)} \frac{d_{\mathrm{A}}}{s}$.

where $\alpha_{F A}$ is the polarizability of the sphere plus adlayer. This equation is valid for $d_{\mathrm{A}} \ll s$. It will be noticed that there is a contradiction between the condition $s \ll \lambda$ used in obtaining equations ( $19-20$ ), and the condition $d_{\mathrm{A}} \ll s$. The first implies that the colloidal particles should have a radius less than $\lambda / 20 \sim 30 \mathrm{~nm}$, which is not much bigger than the value of $d_{\mathrm{A}}$ expected from proteins $\sim$ IO $\mathrm{nm}$ in diameter (and which, moreover, may lead to the complications in the adsorption behaviour referred to earlier ${ }^{3}$ ). For larger particles the full Mie theory should be used to obtain equations corresponding to equations ( 19$)$ to (20); numerical simulations show, however, that the error introduced through using equation (19) may be less than typical experimental errors (Uzgiris \& Fromageot, 1976).

Sedimentation. In this classic method, the motion of particles moving in a strong gravitational field $g$ is observed. Once steady state (velocity $v$ ) has been reached, equating the gravitational force with the frictional force yields:?

$v_{\mathrm{F}}=2 s^{3}\left(\rho_{\mathrm{F}}-\rho_{\mathrm{C}}\right) g /(9 \eta)$.

From the difference $v_{\mathrm{FA}}-v_{\mathrm{F}}$, the increase $d_{\mathrm{A}}$ in particle radius can be deduced, provided that $\rho_{\mathrm{FA}}$ is known, e.g. from allowing the particles to equilibrate in a density gradient.

\subsection{Capillarity}

According to the well-known Poiseuille's law, the product of flow rate $\mathscr{F}$ and viscosity $\eta$ of a Newtonian liquid flowing through a capillary of radius $R$ is proportional to $R^{4}$. When proteins are deposited on the walls of the capillary, $R$ obviously decreases and, provided that $R$ is suitably chosen, a large change in flow may result (Fig. $7 b$ ). The working equation is

$\frac{\eta_{\mathrm{C}}^{\prime}}{\eta_{\mathrm{C}}} \frac{\mathscr{F}_{\mathrm{C}}^{\prime}}{\mathscr{F}_{\mathrm{C}}}=\frac{\left\|R-d_{\mathrm{A}}\right\|^{4}}{\|R\|^{4}}$

where $\|R\|$ is the root mean fourth average defined by:

$\|R\|^{4}=\frac{1}{N} \sum_{1}^{N} R_{i}^{4}$

$\eta_{\mathrm{C}}^{\prime}$ the viscosity of the protein solution, and $\mathscr{F}_{\mathrm{C}}^{\prime}$ the flow rate in the presence of an adlayer. In practice, not a single capillary but a sintered disk containing $N$ capillaries is used (Rowland \& Eirich, I $966 a$ ). There are a number of practical difficulties associated with the evaluation of $d_{\mathrm{A}}$ from $\mathscr{F}$. For example, the

\footnotetext{
7 This equation is a simplification of the exact expressions of Alexandrowicz \& Daniel (1963); moreover the work of these authors preceded the refinement of the simple Stokes-Einstein expression for the friction due to adlayers formed from discrete subunits (see above, equations $13-14$ ).
} 
capillaries will usually be characterized by a distribution of radii $R$. Nevertheless, useful results could be obtained with polymers by Rowland \& Eirich (1966b). They compared the isotherms obtained by the capillarity technique with others obtained by the simple solution depletion method $(\$ 2)$ to determine both density and thickness of the adsorbed layers. The method was later applied to protein adsorption by de Baillou et al. ( 1984 ).

\section{ELECTRICAL METHODS}

When a protein dissolves in an aqueous solution the ionizable amino acids (to which the solution is accessible) in the peptide chain will either lose or acquire a proton and the molecule will become charged. An average globular protein may have dozens of such groups. ${ }^{8}$ The adsorption of proteins at an interface therefore implies the accumulation of charge there; the methods discussed in this section aim to measure this charge. If the total accumulated charge were proportional to the number of proteins, then, knowing the net charge per protein molecule, $\Gamma$ or $\theta$ could immediately be calculated. Therein lie both the difficulties and advantages of electrical methods. In the case of mass the correspondence between the measured attribute (total adsorbed mass) and $\Gamma$ is exact; however, in the case of charge the correspondence is more compiicated. The solid-solution interface is notoriously complex, and hundreds of monographs have been devoted to this topic alone. The value of electrical methods is that they may be used, in conjunction with other techniques providing an independent estimate of $\Gamma$, in order to yield detailed information about the electrostatics of the adsorption process. Another advantage arises from the requirement that adsorption takes place on electrodes: redox operations on proteins and their prosthetic groups can be carried out in situ, and the resultant changes in absorption kinetics, conformation, packing density etc. can be observed directly (Lecompte et al. 1984). ${ }^{9}$ Electrostatic interactions are important because many of the surfaces to which proteins are adsorbed are, like the proteins, ionized in contact with aqueous solutions (Healy \& White, 1978). Even non-ionizable surfaces may acquire a charge through the adsorption of small ions from solution. Electrostatic forces may, therefore, play a key rôle in protein transport to and adhesion at surfaces.

Two basic strategies have been applied to electrical methods. In the first, the

\footnotetext{
- There is of course enormous variety between different proteins; this figure is only mentioned to give an order-of magnitude idea; large extracellular matrix proteins with molecular weights approaching a million may have hundreds of charged groups. At the isoelectric point the net charge $q_{p}$ will be zero, but there are usually patches of oppositely signed charge on the protein's surface. Elsewhere, at all but extremes of $\mathrm{pH}, \Sigma\left|z_{j}\right| \gg \Sigma z_{j}=q_{p}$, where $z_{j}$ is the charge of the $j$ th residue. This may have dramatic consequences on the shape of the molecule (e.g. Rocco et al. 1987). Unfortunately data on the charge of proteins over the physiologically interesting $\mathrm{pH}$ range is rather sparse. Far fewer have been characterized electrically than structurally; often only the isoelectric point is known (Righetti et al. 1981 ). Various formulae have been proposed to predict the net charge of a protein at any given $\mathrm{pH}$ from the $\mathrm{pK}_{a} \mathrm{~s}$ of the constituent amino acids (see the discussion by Kuramitsu \& Hamaguchi, 1980), although it is uncertain how generally reliable such procedures are. As far as the interactions between a protein molecule and the electrical double layer are cuncerned, in the following discussion the protein will be considered to be a point polyion, rather than a small spheroid with its own surface and possessing itself an electrical double layer. The latter case has been adequately handled in the sphere of colloid chemistry (Verwey \& Overbeek, 1948).

${ }^{9}$ Ellipsometry also permits the use of metallic substrates $(\$ 6.1)$.
} 
perturbation of the charge distribution at the interface due to adsorbed proteins is analysed empirically. In the second, the accumulated charge at the solid surface $Q_{\mathrm{A}}$ per unit area is measured and the surface excess deduced from

$\Gamma=Q_{\mathrm{A}} / q_{p}$

where $q_{p}$ is the net charge per protein. The second approach is the simpler; however, if small ions are concomitantly taken up by the interface when the protein is adsorbed, as seems to be a fairly general feature (Norde \& Lyklema, 1979; van Dulm et al. 1981), the simple equation (24) will no longer hold, and the first strategy must be followed. It is probably an understatement to remark that difficulties will attend this approach.

Basic properties of an electrified interface. If some of the groups on the surface (supposed to be planar) of the solid are ionized, or ions of one sign are preferentially adsorbed from the electrolyte solution, the surface (subscript 0 ) will be characterized by a charge density $\sigma_{0}$ and potential $\psi_{0}$. Specifically, in the case of metal and semi-conductor oxides, which become hydroxides in water, the charge usually arises through ionization of the $-\mathrm{OH}$ groups, i.e. through the equilibria (Healy \& White, 1978):

$\mathrm{M}-\mathrm{OH}_{2}^{+} \rightleftharpoons \mathrm{M}-\mathrm{OH}+\mathrm{H}^{+}$

and

$\mathrm{M}-\mathrm{OH} \rightleftharpoons \mathrm{M}-\mathrm{O}^{-}+\mathrm{H}^{+}$,

where $\mathrm{M}=\mathrm{Si}, \mathrm{Ti}, \mathrm{Ta}, \mathrm{Al}$, etc. Protons are thus the potential determining ions of oxides, as well as of phospholipid membranes.

The requirement of overall electrical neutrality ensures that counterions are present, and, were thermal agitation absent, they would arrange themselves in a plane parallel to and at a distance $d_{\varepsilon}$ from the surface, $d_{\epsilon}$ being of the order of the size of the ions, the system thus forming a parallel plane condenser. In the absence of specific counterion association with the surface, however, thermal agitation disperses the mobile counterions into a diffuse layer (Fig. 9). Using the Poisson-Boltzmann equation Gouy (1910) and Chapman (1913) derived the relationship between $\psi_{0}$ and $\sigma_{0}$ :

$\sigma_{0}=\sqrt{ }\left(\epsilon k T c_{b}\right) 2 \sinh \left(e \psi_{0} /(2 k T)\right)$,

where $c_{b}$ here refers to the bulk ion concentration. This relation is valid for $1: \mathbf{I}$ electrolytes. The decrease of potential perpendicular to the surface is given by:

$\tanh (e \psi(z) /(4 k T))=\tanh \left(e \psi_{0} /(4 k T)\right) \exp \left(-z / W_{d d l}\right) ;$

the measure of the decrease is the Debye screening length $W_{d d l}$, given by

$W_{d d l}^{2}=\epsilon k T /\left(2 \sum q_{j}^{2} c_{j}\right)$.

It is this quantity, rather than the Bjerrum length $e^{2} /(4 \pi \epsilon k T)$, which gives the characteristic length of electrostatic phenomena in the presence of mobile ions. In a decimolar solution, the surface potential is screened within less than $1 \mathrm{~nm}$ 


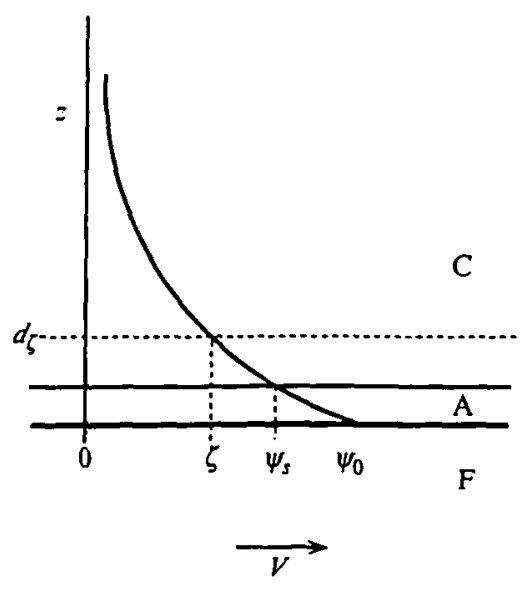

(a)

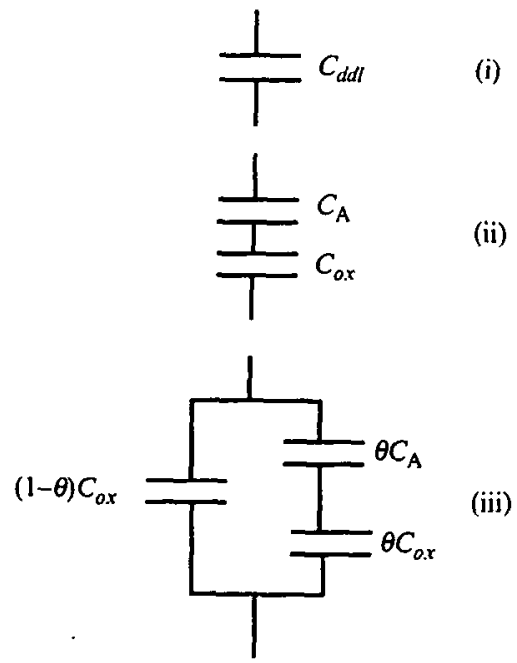

(b)

Fig. 9. (a) Potential distribution perpendicular to the solid-liquid interface. The bulk potential $(z \rightarrow \infty)$ is zero. The potential at the surface of $F$ is $\psi_{0}$ and at the surface of the adlayer, $\psi_{x} . \zeta$ is the potential at the so-called slipping plane dividing the moving from the stationary liquid. (b) (i) Equivalent circuit of the diffuse double layer. (ii) Equivalent circuit of a protein adlayer on a layer of insulating oxide coating a"metal electrode. (iii) As (ii), taking account of the discontinuity of the protein film.

(Gouy, 1910). Thus $W_{d d l}$ may be comparable to, or smaller than, the characteristic size of a protein molecule, $d_{\mathrm{A}}$. While this is explicitly taken account of in the $z$ direction, it means that the lateral distribution of charged species is electrically non-uniform, which can result in discrepancies between experimental results and predictions made on the basis of uniformity (see e.g. Cohen \& Radke, I991). This constitutes an important difference between electrical and optical methods, for which the characteristic length is the wavelength of light-(see $\S 6$ ), i.e. $\lambda \gg d_{\mathrm{A}}$.

Once the potential distribution $\psi(z)$ is known the concentration of ions at any point in the solution can be found using a Boltzmann expression:

$c(z)=c_{b} \exp (-q \psi(z) /(k T))$,

where $q$ is the charge of whatever species we are considering. This equation has an important implication for the $\mathrm{pH}$ at surfaces for which protons are potential determining ions. Let us define a surface $\mathrm{pH}, \mathrm{pH}_{0}$, as $-\log _{10} h_{0}$, where $h$ denotes proton activity. According to (29),

$p H_{0}=-\log _{10}\left(h_{b} \exp \left(-e \psi_{0} /(k T)\right)\right.$.

The point of zero charge of $\mathrm{SiO}_{2}$ is around $\mathrm{pH}$ 2, and of $\mathrm{TiO}_{2}, \mathrm{pH}_{5}-6$ (Hunter, I $98 \mathrm{I})$. These and similar oxide surfaces are therefore expected to be negatively charged at physiological $\mathrm{pH}$. Equation (30) shows that the surface $\mathrm{pH}$ of both oxides and other biologically relevant surfaces (e.g. lipids) may be a couple of units below bulk solution $\mathrm{pH}$. 
The capacity $C_{d d l}$ of the diffuse double layer is $\sigma_{0} / \psi_{0}$. If $e \psi_{0} \ll 2 k T$, the hyperbolic function in (26) can be linearized to yield

$C_{d d l}=\sigma / \psi_{0}=\epsilon / W_{d d l}$,

i.e. charge and potential are proportional to each other and the double layer can be represented as a parallel plate condenser, one plate of which is the solid electrode and the other the diffuse portion of the double layer, represented by a plane at distance $z=W_{d d l}$ from the solid-liquid interface.

Although the Gouy-Chapman formalism has many apparent shortcomings (such as neglect of the size of the ions, and of the variation of $\epsilon$ near the surface) it works remarkably well, for the liquid mercury electrode in particular. The presence of a layer of specifically adsorbed counterions (Stern-Hamburg, I 924; Ermakov, 1990), layers of ordered water (Bockris et al. r 963), etc. complicate the description of the interface. These and other complications can be explicitly taken into account, but usually not without the introduction of parameters whose values cannot be independently determined, and the resulting systems of equations are no longer convenient to handle.

\subsection{Impedance measurements}

An electrochemical cell is set up consisting of a working electrode on which adsorption is to take place, a reference electrode, and a counter electrode. The working electrode should be ideally polarizable so that no faradaic currents flow, and its impedance should be much greater than that of the counter electrode. The potential difference between the working and counter electrodes is adjusted such that the potential difference between the reference and the working electrodes remains constant. Under these conditions the impedance of the cell, which can be measured using standard methods (Bard \& Faulkner, 1 980), is that of the working electrode at an absolute electrochemical potential $\psi_{0}$. It has been found that the adsorption of proteins on its surface causes the capacity to decrease (Lecompte $e t$ al. 1984). If an empirical Frumkin-type equation is used:

$C=C_{0}(\mathrm{I}-\theta)+\theta C_{1}$,

where $C_{0}$ is the capacity of the bare electrode, and $C_{1}$ the capacity after saturating the surface with protein, $\theta$ can be determined. This method can only be considered approximate. The range of validity of the assumptions that $C_{1}$ corresponds to $\theta=\mathrm{I}$, and that $C$, presumably corresponding to $C_{d d l}$ (equation $3 \mathrm{I}$ ) varies linearly with $\theta$, are generally unknown. However, it may be the best we can do, for the explicit relationship in equation (26) between $\psi_{0}$ and $\sigma_{0}$ is only valid for the liquid mercury electrode (equivalent circuit shown in Fig. $9 b$ (i)).

Liquid mercury has only limited interest as a substrate on which to adsorb proteins. Solid metals can also be used but impurities and unevenness of the surface complicate the impedance (Stoner \& Srinavasan, I970), apart from the fact that most metals are not ideally polarizable and the standard treatment of the interface does not apply, although this will not necessarily affect the use of an 
empirical adsorption isotherm. The use of a rotating disk electrode (Bernabeu et al. 1989) is an important extension which allows experiments to be carried out under precisely defined and controllable hydrodynamic conditions (Levich, 1962).

A slightly more elaborate setup has been investigated by Gebbert et al. (1992). The working electrode (tantalum) was covered by a thin, but insulating, layer of tantalum oxide ${ }^{10}$ (to which specific protein ligands (antibodies) were attached). The capacitance $C_{\text {o.x }}$ of the insulating oxide layer $\left(\sim \mathrm{I} \mu \mathrm{F} / \mathrm{cm}^{2}\right)$ is much smaller than $C_{d d l}\left(\sim 40 \mu \mathrm{F} / \mathrm{cm}^{2}\right)$ and the latter can hence be neglected. The formation of a layer A of adsorbed protein is supposed to result in a capacitance $C_{\mathrm{A}}$ in series with $C_{o x}$ (Fig. $9 b(\mathrm{ii}))$. The measured cell capacitance is then

$C=C_{o x} C_{\mathrm{A}} /\left(C_{o x}+C_{\mathrm{A}}\right)$.

Knowing $C_{o x}$ from prior measurement on the clean electrode, $C_{\mathrm{A}}$ may then be determined from $C$. There is, however, no explicit equation connecting $C_{\mathrm{A}}$ with $\Gamma$ or $\theta$, although one might guess at a relation of the type

$C_{\mathrm{A}}=\epsilon_{A} / d_{\mathrm{A}}$.

Unless the protein forms a homogeneous, insulating layer, however, the electrolyte solution establishes an equipotential surface in contact with the oxide layer everywhere except where a protein molecule is adsorbed. Fig. $9 b$ (ii) must be replaced by the equivalent circuit in Fig. $9 b$ (iii), and equation (33) by the corresponding measured cell capacitance:

$C=(\mathrm{I}-\theta) C_{0 x}+\theta C_{0 x} C_{\mathrm{A}} /\left(C_{o x}+C_{\mathrm{A}}\right)$.

This equation has two unknowns, $\theta$ and $C_{\mathrm{A}}$, which may be determined by measuring the impedance of protein adsorbed on oxide layers of two different thicknesses in order to evaluate both $\theta$ and $C_{\mathrm{A}}$ (Stenberg et al. 1979). These workers found, using such a technique, that $C_{\mathrm{A}}$ was roughly proportional to $\theta$, although the proportionality may depend on the particular protein, $\mathrm{pH}$, and salt conditions used. Therefore, use of the simpler equation (33) appears to be justified, and could be employed to determine $\theta$; it may be speculated that $d_{\mathrm{A}} \propto \theta$.

Semiconductor electrodes. The use of semiconductor electrodes in principle allows more information to be obtained from impedance measurements than is possible with metallic electrodes. Fig. Io $a$ shows, for a moderately doped $n$-type semiconductor, the energy bands when the surface and bulk electrochemical potentials are equal. The surface potential is supposed to be the same as the electrochemical potential of the bulk electrolyte, which is determined by the potential difference between the counter and working electrodes, or by a suitable redox buffer (Reinders \& de Minjer, 1938). If this surface ( $\equiv$ bulk electrolyte) potential is lowered slightly by an amount $V_{d}$, equilibrium must be reestablished by electron exchange between the solution-surface domain and the semiconductor. Conduction band electrons will move out of the bulk semiconductor until the electrochemical potentials are again equal. The situation

10 Stelzle and Sackmann (1989) have carried out a similar experiment using a lipid bilayer in place of the oxide. 


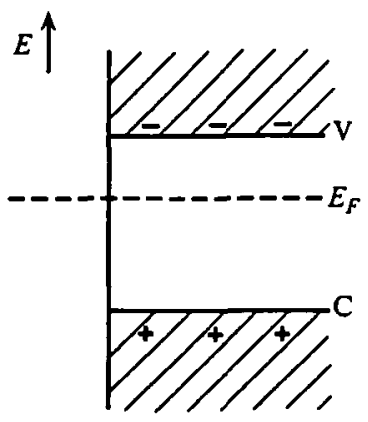

$\overrightarrow{I=1}$

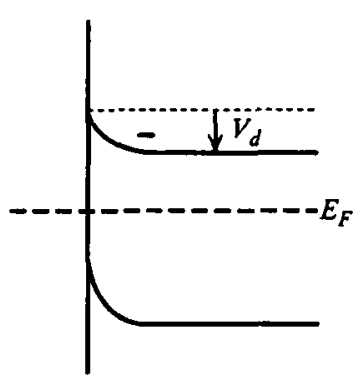

(b)
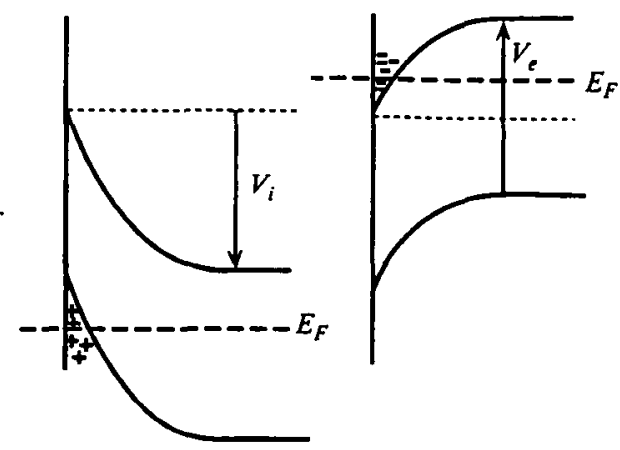

(c)

(d)

Fig. 10. Energy bands in an $n$-type semiconductor. (a) At equilibrium. The electrochemical potential of the electrolyte equals that of the electrons in the semiconductor (the Fermi level, $E_{F} . \mathrm{V}$ and $\mathrm{C}$ denote respectively the valence and conduction bands of the semiconductor. (b) Bands bent by the application of a potential difference $V_{d}$ between the surface and the bulk of the semiconductor. The reequilibration of the electrochemical potentials within and without the semiconductor requires electrons to flow from the bulk of the semiconductor to the electrolyte and hence a depletion layer is created (region of band bending). (c) The application of a potential $\left|V_{l}\right|>\left|V_{d}\right|$ results in such severe band bending that positive holes (defect electrons) accumulate at the surface. The surface conductivity of the semiconductor thus depends on the minority carriers. (d) Application of a potential of opposite sign to that of $V_{d}$ creates an enrichment zone of majority carriers at the surface.

is analogous to the metal-semiconductor contact (Schottky, 1942). These electrons must come from donors, which are ionized and form stationary positive charges, resulting in a space charge region adjacent to the surface in which the conduction band is heavily depleted of electrons, with width: ${ }^{11}$

$W_{s c}=\sqrt{ }\left[2 \epsilon_{s c} V_{d} /\left(e^{2} N_{D}\right)\right]$.

This equation may be compared with (28). Analogously to the diffuse double layer in the electrolyte (equation 31 ), the capacitance per unit area is given by

$C_{s c}=\epsilon_{s c} / W_{s c}$.

Typically $C_{s c} \sim \mathrm{I} \mu \mathrm{F} / \mathrm{cm}^{2}$ and the equivalent circuit of the electrochemical cell reduces to a single condenser (Fig. I $b$ ) as in Fig. $9 b$ (i). If the surface potential is changed by the adsorption of charged proteins, $V_{d}$ must be replaced by $V_{d}+V_{p}$, where $V_{p}$, the potential due to adsorbed proteins, is given by:

$V_{p}=Q_{\mathrm{A}} / C_{\mathrm{A}}$.

Knowing the charge $q_{p}$ per protein, the measurement of the change in impedance of the semiconductor space charge allows $\Gamma$ to be determined from equation (24). If neutral proteins bearing a dipole moment $\mu_{p}$ are adsorbed, then

11 Here and in the following, the equations have been somewhat simplified. The full treatment can be found in standard textbooks, e.g. Myamlin \& Pleskov, 1967; Pleskov \& Gurevich, 1986). 
$V_{p}=\Gamma \mu_{p} /\left(C_{\mathrm{A}} d_{\mathrm{A}}\right)$.

A difficulty is that $C_{\mathrm{A}}$ is not necessarily known, and indeed, as mentioned above, may be proportional to $\Gamma$. Moreover, $C_{\mathrm{A}} \approx C_{s c}$, and therefore the additional capacitance $C_{\mathrm{A}}$ should be added in series with $C_{s c}$. Impedance measurements on two electrodes differing only in their doping density $N_{D}$ would allow $C_{\mathrm{A}}$ and $C_{s c}$ to be determined, and hence $\Gamma$. Fig. I I $a$ shows schematically the measuring set-up. The application of such measurements to protein adsorption does not, however; appear to have been reported. A general problem is that the real equivalent circuit of a semiconductor electrode is vastly more complicated than is suggested by Fig. i $b$ (Gobrecht $\&$ Meinhart, I 963 ; Harten, I 964 ; Boddy, I 965 ; Myamlin \& Pleskov, 1967; Pleskov \& Gurevich, 1986), even with judicious choices of measurement frequency and $V_{d}$. The determination of the parameters from an impedance spectrum can be made by fitting to equivalent circuits of arbitrary complexity, but unless there is an independent physical basis for the chosen circuit, the procedure evidently has little value.

The effect of accumulation of charge from adsorbed proteins at the surface of a semiconductor electrode is exploited in another kind of device, the field effect transistor or FET (Sze, 1981), in which the lateral current between two electrodes embedded in the semiconductor surface is measured (Fig. I I $c$ ). The conductivity $K_{\text {subs }}$ of the subsurface layer is

$K_{\text {subs }}=e n \mu$,

where $\mu$ is the carrier mobility and $n$ the concentration of carriers in the subsurface layer, which in turn is given by

$n=n_{i}+N_{D} \exp \left(e V_{d} /(k T)\right)$,

$n_{i}$ being the intrinsic carrier concentration of the undoped semiconductor. Fig. Io shows the various possible régimes for an $n$-type semiconductor. In (c), the applied voltage $V=V_{i}$ is sufficiently negative to drive the interface into inversion and current is mainly carried by defect electrons (positive holes); in $(d)$ an enrichment layer is formed by an applied potential of the opposite polarity. In the inversion region the current $I_{D}$ from the source to the drain at a given source-drain potential $V_{D}$ (Fig. I I $c$ ) depends approximately linearly on the voltage $V_{p}$ due to protein adsorption (equation 38 ) and the preset gate voltage $V_{G}($ Sze, I $98 \mathrm{I})$ :

$I_{D} \approx \frac{W}{L} \mu C_{o x}\left(V_{G}+V_{p}-V_{T}\right) V_{D}, \quad V_{D} \ll\left(V_{G}+V_{p}-V_{T}\right)$,

where $V_{T}$, the threshold voltage, is given by:

$V_{T}=2 \psi_{B}+\sqrt{ }\left(4 \epsilon_{s c} e N_{D} \psi_{B}\right) / C_{o x}$

( $\psi_{B}$ is the difference between the actual and intrinsic Fermi levels) and $L$ and $W$ are respectively the length and width of the channel. Therefore, $V_{p}$ and hence $\Gamma$ can be calculated from a measurement of $I_{D}$ as in the impedance measurements above. Attempts been made to apply such devices to analytical problems in 


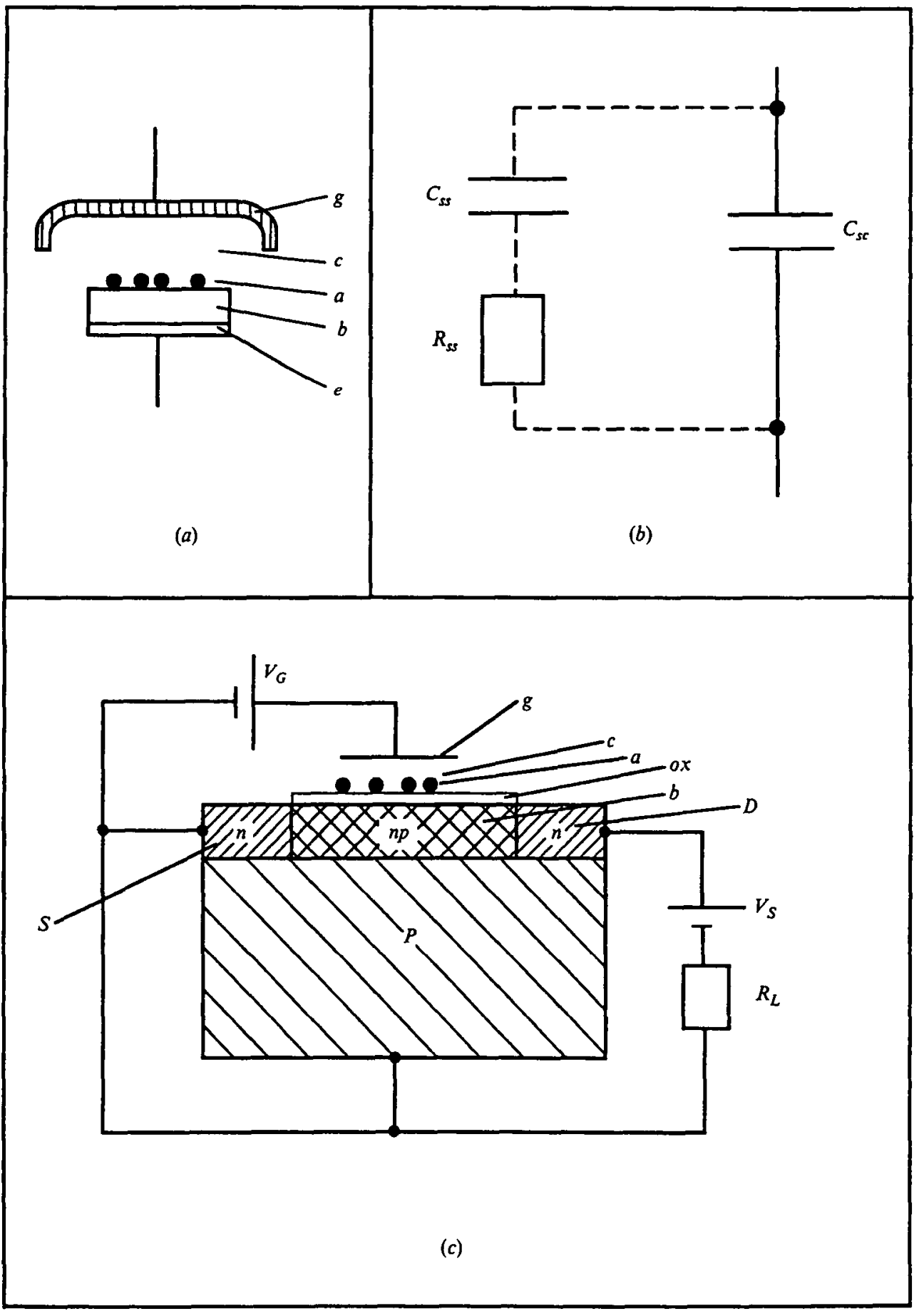

Fig. Ir. (a) Scheme of cell for measuring the impedance of a semiconductor electrode. $a$, adsorbed proteins; $b$, bulk semiconductor; $c$, cover medium (electrolyte solution); $e$, ohmic contact; $g$, counterelectrode. Practical impedance measurements of semiconductor junctions are discussed by Popkirov \& Tabov (1982). (b) Simplified equivalent circuit of a semiconductor electrode in depletion (corresponding to Fig. $10 b$ ). $C_{s c}$ is given by equations 36 and 35 . Subscripts ss refer to surface state equivalent impedances which must be taken 
aqueous solutions (Caras \& Janata, I988). A lot of work has been put into them because they offer the possibility of miniaturized immunoassay devices which can be interfaced directly to sophisticated, integrated circuitry, and considerable success has been achieved with ion-sensitive FETs (ISFETS). For this type of application, it is not necessary to convert $I_{D}$ into $\Gamma$. A problem with all these semiconductor devices is the chemical reactivity of the semiconductor surface in the presence of water (Gobrecht \& Meinhart, 1963; Efimov \& Erusalemchik, 1963), which results in the formation of hydrated oxide layers at the surface, and the response drifts with time.

Electrical measurement of adsorption on lipid membranes. Bilayer lipid membranes are slightly compressible; compression can be induced by applying an electrical potential across the membrane. Since capacitance is inversely proportional to thickness, membrane capacitance is a function of transmembrane potential (Alvarez \& Latorre, 1978):

$C_{\mathrm{M}}=C_{\mathrm{M}, 0}\left(\mathrm{I}+\alpha V_{p}^{2}\right)$

assuming here that the membrane potential arises solely through the adsorption of proteins on one side of a symmetric bilayer. $C_{\mathrm{M}, 0}$ is the capacitance in the absence of a transmembrane potential. If a sinusoidally varying potential is now applied to the bilayer lipid membrane the current response will show second order harmonics whose magnitude is proportional to $V_{p}$ due to the nonlinearity of the impedance (Cherny et al. 1980). Measurement of the amplitude of the second harmonic (or, better, the applied potential $-V_{p}$ required to make it disappear) is a sensitive way of determining $V_{p}$. The technique is then called the inner field compensation method (IFCM). $\Gamma$ is calculated from $V_{p}$ using equations (38) and (24). ${ }^{12}$ If $V_{p}$ is determined using the null balance technique, it is not necessary to know the constant $\alpha$; otherwise $\alpha$ could be determined by measuring $V_{p}$ for a series of asymmetric membranes incorporating small numbers of ionizable lipids (e.g. a mixture of phosphatidylcholine and phosphatidylglycerol) on one side. The method was successfully applied to measure the kinetics of the adsorption of synthetic polyions to phosphatidylcholine membranes (Ermakov et al. I985), and there appears to be no reason for not applying it to protein adsorption problems, although no results have to date been reported.

into account at low measuring frequencies. (c) Insulated gate (metal oxide) field effect transistor (MOSFET) adapted to measuring adsorption from solution. $a$, adsorbed proteins; $b$, bulk semiconductor (conducting channel); $c$, cover medium (electrolyte solution); $d$, drain electrode; $g$, counterelectrode; $o x$, oxide insulating layer; $s$, source electrode. $n, p$, and $n p$ refer to the doping of the various regions of the bulk semiconductor. $V_{G}$ is the potential applied between the oxide surface and the semiconductor bulk. $V_{s}$ is the source-drain potential. The drain current $I_{D}$ is measured across the load resistance $R_{L}$.

${ }^{12}$ In principle, the same technique can be applied to the semiconductor electrode - with or without a lipid membrane - which is preferably biased before the addition of protein to the system such that no higher harmonics appear, a condition which, it may be added, is almost impossible to achieve because additional nonlinear impedances such as surface states are also present. An attempt has, nevertheless, been made to apply the method to study the kinetics of protein adsorption onto a platinum electrode (Nakata et al. 1992), without it having been possible to evaluate $\Gamma$, however. 


\subsection{Electrokinetic methods}

In the cells we have considered in the preceding section, no special attention has been paid to motion of the liquid phase relative to that of the solid. If such motion is caused to occur, then the relative velocity will increase continuously from zero at the interface to a maximum value far from it. In treating the electrostatics of the problem, the approximation is usually made that from $z=0$ to $d_{\xi}$ the velocity is zero, and for $z>d_{\xi}$ it has its greatest velocity. The solid thus behaves as a body with a potential $\zeta$ relative to the bulk liquid phase (Fig. 9); the problem of electrokinetics is firstly to measure $\zeta$ and secondly to calculate $\psi_{0}$ from $\zeta$ (Hunter, I98I). If a potential difference $E$ is applied parallel to the interface, a force $F_{1}=$ $\sigma E$ appears, where $\sigma=\zeta \epsilon / d$ and $d$ is the distance separating the two phases, which tends to displace one phase relative to the other with velocity $\mathbf{u}$. This force is opposed by a frictional force $F_{2}=-\eta \mathrm{d} u / d \lambda$, where $\eta$ is the kinematic viscosity of the liquid, and $\lambda$ the thickness of the liquid layer in which the velocity is changing. After a short time a steady state is reached, i.e. $F_{1}=-F_{2}$, and supposing $d u / d \lambda=$ $\mathbf{u} / \lambda$ and $\lambda=d$, we obtain

$\mathbf{u}=\epsilon \zeta \mathbf{E} / \eta$

the Helmholtz-Smoluchowski equation, the basic equation underlying all electrokinetic phenomena. ${ }^{13}$

Electrophoresis. The solid phase is in the form of uniform colloidal spheres or liposomes, suspended in a liquid, and accelerated by an applied electric field. The steady state velocity is observed either through a microscope or via quasi-electric light scattering. ${ }^{14}$ Various corrections have been applied to equation 45 (Wiersema et al. 1 966; O'Brien \& White, 1978; Mangelsdorf \& White, 1990). Electrophoresis alone is not suitable for measuring $\Gamma$ because the electrophoretic mobility $\mathbf{u} / \mathbf{E}$ depends on both the friction coefficient, which changes with $d_{A}$ (equations 12-14), and on $\zeta$, which changes with $Q_{\mathrm{A}}$ (equation 26), and a unique solution for $\Gamma$ is not possible.

Streaming potential. The liquid phase is driven by pressure $P$ along a conduit, which we suppose to have the cross section of a rectangular prism of height $H$ much smaller than its width and length. As a result, a streaming potential $V_{\text {str }}$ is measured, which is related to $\zeta$ according to (van Wagenen \& Andrade, 1980):

$\zeta=\left(K_{b}+2 K_{0} / H\right) 4 \pi \eta_{\mathrm{C}} V_{s t r} /(P \epsilon)$,

where the $K$ are bulk (subscript $b$ ) and surface (subscript 0 ) conductances. Van Wagenen \& Andrade (1980) maintain that $K_{0}$ is negligible, and the other parameters can be determined independently. Therefore, $\zeta$ can be converted to $\psi_{0}$, using equation (29), $\psi_{0}$ after adsorption subtracted from $\psi_{0}$ before adsorption to

19 Either the solid or the liquid may be stationary, and either $\zeta$ engendered by an applied force or the velocity engendered by an applied electric field may be measured.

${ }_{14}$ See $\$ 4.1$. The phase auto-correlation function (equation 18 ) then equals exp (iK.ur) Cummins \& Swinney, 1970). This possibility is of particular interest, because initially the hydrodynamic radius of the colloidal substrate can be determined in the absence of an applied electric field and in the absence of protein. As adsorption proceeds, the autocorrelation functions in the absence and presence of an applied electric field can then be recorded, to allow the relationship between $d_{A}$ and $Q_{A}$ to be examined in detail. 
yield $V_{p}$, and $V_{p}$ converted to $\Gamma$ using equations (38) and (24). Absolute determinations of $\Gamma(t)$ have not, however, been reported; in practice too few of the necessary parameters are known independently to make the calculation reliable. Norde \& Rouwendal (1990) first measured the adsorption isotherm using a solution depletion technique, obtained an empirical relation between $\zeta$ and $\theta$ by measuring the electrophoretic mobility of the adsorbent particles, and then used the streaming potential measurement to determine $\theta$ in real time.

\section{OPTICAL METHODS}

Elements of catoptrics. When a ray of light is incident upon the plane separating two media of different refractive indices, ${ }^{15}$ the ratios of the reflected and incident amplitudes are given by the well-known Fresnel equations $:^{16}$

$\mathscr{R}_{s}=A_{r, s} / A_{i, s}=-\sin \left(\theta-\theta^{\prime}\right) /\left(\sin \left(\theta+\theta^{\prime}\right)\right)$

and

$\mathscr{R}_{p}=A_{r, p} / A_{i, p}=\tan \left(\theta-\theta^{\prime}\right) /\left(\tan \left(\theta+\theta^{\prime}\right)\right)$

for light polarized perpendicularly (subscript $s$, senkrecht) and parallel (subscript $p$ ) to the plane of reflexion. $\theta$ is the angle between the incident light and the normal to the planar boundary separating the two materials, and $\theta^{\prime}$ the corresponding angle of the refracted beam. The reflected beam makes an angle $\theta$ with the normal (Fig. I2). $\theta^{\prime}$ can be eliminated from these equations by using Snell's law:

$\sin \theta^{\prime}=\left(n_{\mathrm{F}} / n_{\mathrm{J}}\right) \sin \theta$,

and, defining two quantities corresponding respectively to the components of the reflected beam parallel and perpendicular to the interface:

$\mathscr{B}=k n_{\mathrm{F}} \sin \theta=k N$

and

$k_{\mathrm{F}}=k n_{\mathrm{F}} \cos \theta=k \sqrt{ }\left(n_{\mathrm{F}}^{2}-N^{2}\right)$,

where $k=2 \pi / \lambda$ is the modulus of the wave vector for light of wavelength $\lambda$, and a third quantity

$k_{\mathrm{J}}=k \sqrt{ }\left(n_{\mathrm{J}}^{2}-N^{2}\right)$,

the Fresnel coefficients can be written as:

$\mathscr{R}_{s}=\left(k_{\mathrm{F}}-k_{\mathrm{J}}\right) /\left(k_{\mathrm{F}}+k_{\mathrm{J}}\right)$

and

$\mathscr{R}_{p}=\left(k_{\mathrm{F}} / n_{\mathrm{F}}^{2}-k_{\mathrm{J}} / n_{\mathrm{J}}^{2}\right) /\left(k_{\mathrm{F}} / n_{\mathrm{F}}^{2}+k_{\mathrm{J}} / n_{\mathrm{J}}^{2}\right)$.

As $\theta$ increases from 0 , at a certain angle given by $\arcsin \left(n_{\mathrm{J}} / n_{\mathrm{F}}\right), \sin \theta^{\prime}$ will equal unity and further increase in $\theta$ results in an imaginary $\theta^{\prime}$. This is the regimen of

15 Here and in what follows $\mathrm{F}$ denotes the optically denser medium in which the beam of light originates. The rarer medium is denoted by $\mathrm{J}$.

${ }^{10}$ These equations can be found in standard texts on optics, such as Jenkins \& White (1950). 


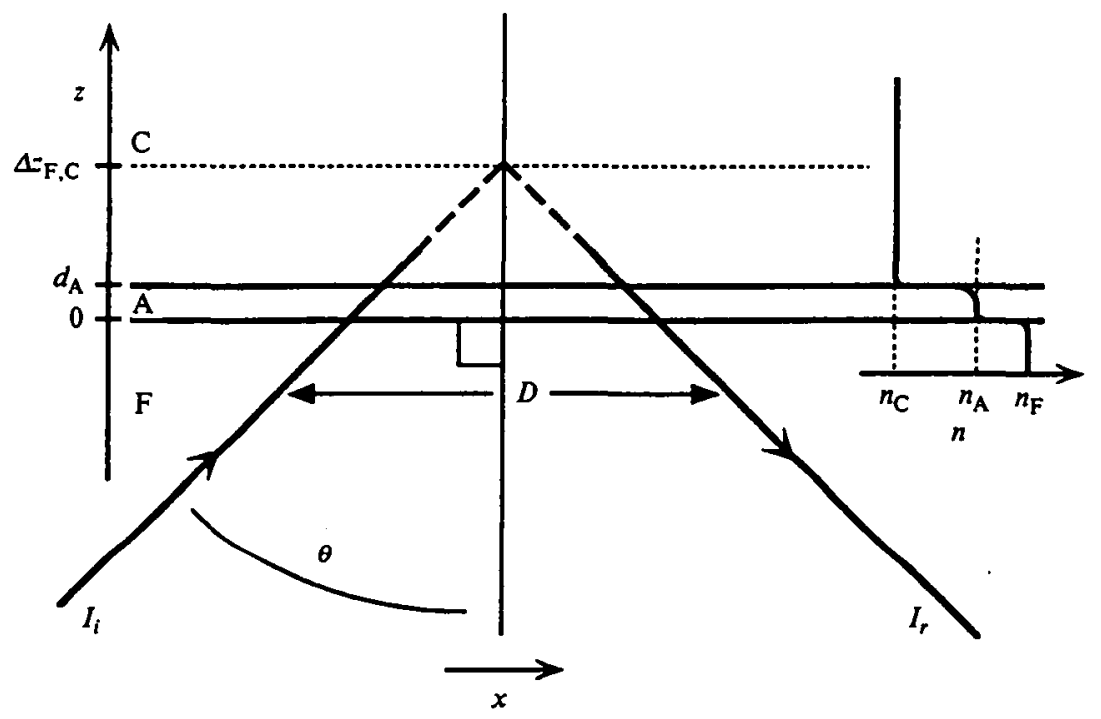

Fig. I 2. Path of a totally internally reflected light beam at the F, C interface between two dielectrics. $A$ is a thin adlayer of adsorbed proteins (its thickness is greatly exaggerated with respect to $\Delta z_{\text {F.c }}$ ). An ellipsometer measures the polarizations of the incident $\left(I_{1}\right)$ and reflected $\left(I_{r}\right)$ beams. Inset: refractive index profile of the interface.

total internal reflexion. The Fresnel coefficients are now complex, with modulus unity since all the energy is reflected, but the phase of the light changes by $\Phi$, i.e.

$R_{F, \mathrm{~J}}=\exp \left(i \Phi_{F, \mathrm{~J}}\right)$.

The attributes of the reflected light are altered by the presence of a layer of adsorbed molecules at the $F, J$ interface. Let the adsorbed layer form a uniform, isotropic layer $\mathrm{A}$ characterized by a geometrical thickness $d_{\mathrm{A}}$ and a concentration within the layer $c_{\mathrm{A}}$ (Fig. $2 b$ ). Since $d_{\mathrm{A}} \ll \lambda$, this interfacial or surface layer constitutes an effective medium with a refractive index profile shown on the extreme right of Fig. 12. The refractive index is related linearly to $c_{\mathrm{A}}$ according to the relation (de Feijter et al. 1978):

$n_{\mathrm{A}}=n_{\mathrm{C}}+c_{\mathrm{A}} \mathrm{d} n / \mathrm{d} c$

which has empirically been shown to hold for homogeneous solutions of proteins up to concentrations of at least $400 \mathrm{mg} / \mathrm{cm}^{3}$ ( $\equiv \Gamma=0.4 \mu \mathrm{g} / \mathrm{cm}^{2}$ ). $c_{\mathrm{A}}$ can be eliminated between equations $(56)$ and $(4)$ to obtain the working equation

$\Gamma=d_{\mathrm{A}}\left(n_{\mathrm{A}}-n_{\mathrm{C}}\right) /(\mathrm{d} n / \mathrm{d} c)$.

When the light coming from $\mathrm{J}$ first meets the F, A plane, it is partly reflected and partly refracted. The refracted beam undergoes multiple partial reflexions at the $A, C$ and $A, F$ interfaces, and the resulting wave reflected back into $F$ is the sum of the partial plane waves:

$\mathscr{R}_{\mathrm{F}, \mathrm{A}, \mathrm{J}}=\mathscr{R}_{\mathrm{F}, \mathrm{A}}+\mathscr{T}_{\mathrm{F}, \mathrm{A}} \mathscr{T}_{\mathrm{A}, \mathrm{F}} \mathscr{R}_{\mathrm{A}, \mathrm{J}} \exp \left(-2 i \beta_{\mathrm{A}}\right)+\mathscr{T}_{\mathrm{F}, \mathrm{A}} \mathscr{T}_{\mathrm{A}, \mathrm{F}} \mathscr{R}_{\mathrm{F}, \mathrm{A}} \mathscr{R}_{\mathrm{A}, \mathrm{J}}^{2} \exp \left(-4 i \beta_{\mathrm{A}}\right)+\ldots$, 
where the $\mathscr{T}$ are the Fresnel transmission coefficients and $\beta_{A}$ is the film phase thickness (i.e. phase change undergone by the beam upon traversing the film) which is

$\beta_{\mathrm{A}}=d_{\mathrm{A}}\left|k_{\mathrm{A}}\right|$,

$k_{\mathrm{A}}$ being defined in the same way as $k_{\mathrm{J}}$ (equation 52 ). If the lateral extent of the films is much greater than their thicknesses, equation $\left(5^{8}\right)$ can be summed to infinity, giving (Vašíček, I960; Azzam \& Bashara, 1977):

$\mathscr{R}_{\mathrm{F}, \mathrm{A}, \mathrm{J}}=\frac{\mathscr{R}_{\mathrm{F}, \mathrm{A}}+\mathscr{R}_{\mathrm{A}, \mathrm{J}} \exp \left(-2 i \beta_{\mathrm{A}}\right)}{1+\mathscr{R}_{\mathrm{F}, \mathrm{A}} \mathscr{R}_{\mathrm{A}, \mathrm{J}} \exp \left(-2 i \beta_{\mathrm{A}}\right)}$,

where $\mathscr{R}_{F, A}$ is given by equations (53) or (54) according to the polarization of the incident beam, with $\mathrm{J}$ replaced by A (similarly for $\mathscr{R}_{\mathrm{A}, \mathrm{J}}$ ). For a second adlayer $B$, the reflexion coefficient $\mathscr{R}_{\mathrm{F}, \mathrm{A}, \mathrm{B}, \mathrm{J}}$ is obtained by replacing $\mathscr{R}_{\mathrm{A}, J}$ in equation (60) by $\mathscr{R}_{\mathrm{A}, \mathrm{B}, \mathrm{J}}$ :

$\mathscr{R}_{\mathrm{A}, \mathrm{B}, \mathrm{J}}=\frac{\mathscr{R}_{\mathrm{A}, \mathrm{B}}+\mathscr{R}_{\mathrm{B}, \mathrm{J}} \exp \left(-2 i \beta_{\mathrm{B}}\right)}{\mathrm{I}+\mathscr{R}_{\mathrm{A}, \mathrm{B}} \mathscr{R}_{\mathrm{B}, \mathrm{J}} \exp \left(-2 i \beta_{\mathrm{B}}\right)}$

and so on for any number of additional adlayers. In the derivation of these results, it is assumed that the layers are homogeneous, isotropic, uncharged and nonconducting, and that the interfaces are smooth and homogeneous. Aspnes (1982) has summarized the effective medium approaches of Maxwell-Garnett, Lorentz-Lorenz and others which have been developed to deal with heterogeneous thin films (see also Strachan, 1933). Charge and conductivity, which are realistic attributes of an adlayer formed from proteins adsorbed from aqueous salt solutions, can be taken into account by using a complex refractive index $n_{A}$ in place of the real one hitherto assumed. The problem has not yet been extensively investigated but representative calculations show that the imaginary part required is very small, and that the error introduced by neglecting it appears to be smaller than the current experimental uncertainty in the determination of the optical constants of the interface (R.-I. Stohn, unpublished results).

Although, under conditions of total internal reflexion, all the incident power is reflected, nevertheless the light travels some way into the rarer medium before returning to the denser one (Newton, 1687). This was demonstrated directly by Goos \& Hänchen (1947, 1950) who showed that upon each reflexion a lateral shift $D$ occurs (Fig. 12). This shift depends upon the angle $\theta$ and the refractive indices of the media according to (Artmann, 1948):

$D=2\left(n_{\mathrm{F}} / n_{\mathrm{J}}\right)^{2 \rho_{n}} n_{\mathrm{J}} k_{\mathrm{J}}$,

where $\rho$ equals $O$ and $\mathrm{I}$ for the $s$ and $p$ polarizations respectively, whence we can define penetration depths $\Delta z$ :

$\Delta z_{\mathrm{F}, \mathrm{J}, \mathrm{s}}=\mathrm{I} / k_{\mathrm{J}}$

and

$\Delta z_{F, J, p}=\mathrm{I} /\left(k_{\mathrm{J}}\left[\left(N / n_{\mathrm{F}}\right)^{2}+\left(N / n_{\mathrm{J}}\right)^{2}-\mathrm{I}\right]\right)$. 
The significance of $\Delta z$ is that the light is reflected not at the F, J interface, but at an imaginary plane situated at a distance $\Delta z_{\mathbf{F}, \mathrm{J}}$ from the interface (Fig. I2). The wave in medium $J$ is evanescent and decays exponentially as $\exp \left(-\Delta z_{\mathrm{F}, \mathrm{J}}\right)$. For visible light reflected at the glass-water interface, the distance $\Delta z$ is typically $\sim 200 \mathrm{~nm}$.

Provided that the index of refraction and thickness of the adsorbed layer can be determined, we can readily calculate the mass or number of molecules adsorbed at the interface. Furthermore, the refractive index and thickness of $\mathrm{A}$ give - in principle - valuable information about the packing density and conformation or orientation of the adsorbed molecules out of which it is constituted (Schaaf \& Dejardin, 1988). At very low $\Gamma$, the experimentally determined $d_{\mathrm{A}}$ and $n_{\mathrm{A}}$ show large fluctuations, which are correlated with one another and which disappear when $\Gamma$ is calculated using equation (57) (Cuypers et al. I983, J. J. Ramsden, unpublished observations). The reason is presumably that when very few proteins are present on the surface, the film boundary is very ill-defined.

\subsection{Ellipsometry}

In the most usual form of this technique, reflexion ellipsometry, polarized light is reflected obliquely from the interface under investigation. The change of elliptical polarization upon reflexion is related to the thickness and refractive index of the adlayer (Azzam \& Bashara, 1977). Let the state of polarization of a beam be characterized by $\mathscr{A}$, the ratio of the amplitudes of the $p$ and $s$ polarizations:

$\mathscr{A}=I_{p} / I_{s}$

and by the phase difference $\mathscr{D}$ :

$\mathscr{D}=\delta_{p}-\delta_{s}$.

$\mathscr{A}$ and $\mathscr{D}$ are measured before and after reffexion at the interface under study, and one may define two angles,

$\psi=\arctan \left(\mathscr{A}_{r} / \mathscr{A}_{i}\right)$

and

$\Delta=\mathscr{D}_{r}-\mathscr{D}_{i}$

the subscripts denoting the incident and reflected beams. From the Fresnel reflexion coefficients one may then derive

$\mathscr{R}_{p} / \mathscr{R}_{s}=\tan \psi \exp (i \Delta)$,

which is the fundamental equation of ellipsometry. A typical procedure (McCrackin et al. 1963) would be to start with the clean interface. The polarizer is adjusted (setting $P$ ) such that the reflected beam is linearly polarized, and can be extinguished by the analyzer (setting $A$ ). Then

$\psi=A$,

$\Delta=2 P+\pi / 2$,

from which the ratio $\mathscr{R}_{p} / \mathscr{R}_{z}$ can be determined. Fig. I 3 shows an example of data obtained using reflexion ellipsometry. 


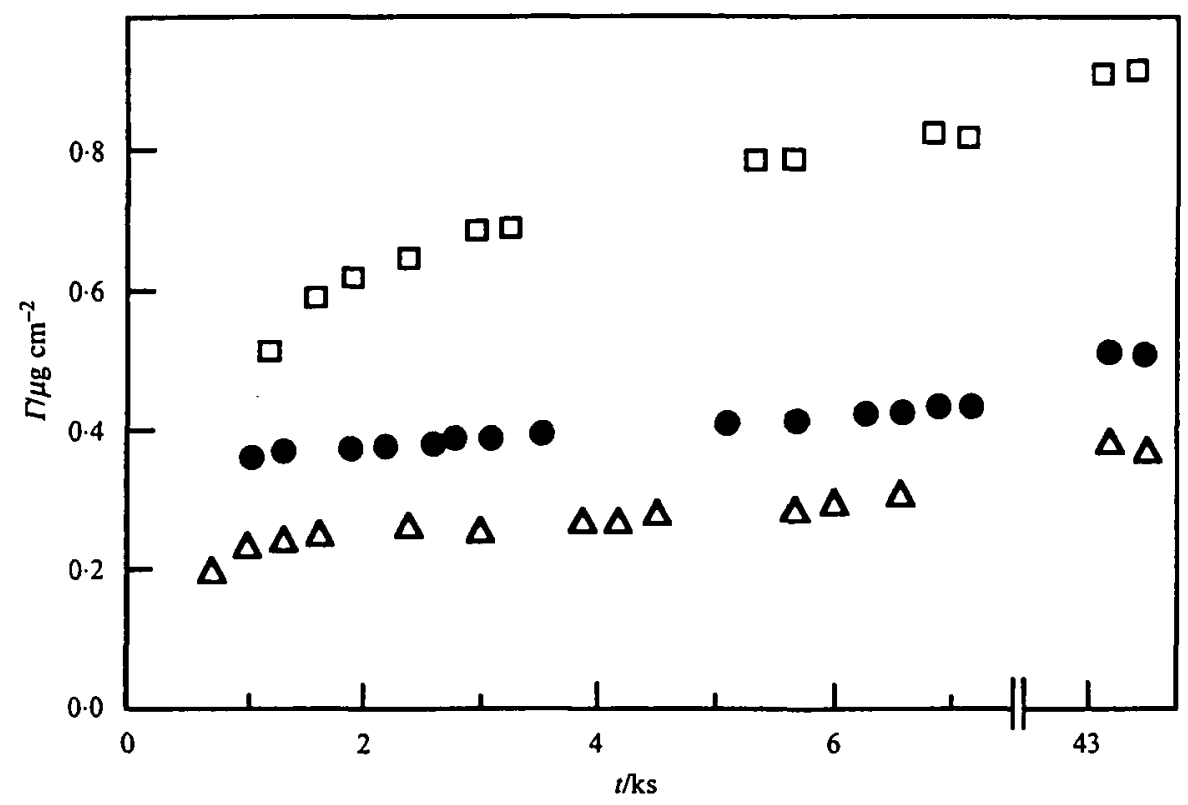

Fig. 13. Kinetics of adsorption of gelatin onto the ( 111 ) face of a silver bromide single crystal measured using reflexion ellipsometry (Maternaghan \& Ottewill, 1974). The gelatin (isoelectric point $\mathrm{pH}_{4} \cdot 8$ ) was dissolved in $10 \mathrm{mM}^{-8} \mathrm{KNO}_{3}$ plus $1 \mathrm{mM}-\mathrm{KBr}$ at a concentration sufficiently high for the adsorption to be independent of it, namely, $\sim \mathrm{r} \mathrm{mg} / \mathrm{cm}^{3} . T=$ $40.0 \pm 0.02^{\circ} . \triangle, \mathrm{pH}_{3.5} ; \square, \mathrm{pH}_{4} 4 ; 0, \mathrm{pH}^{\circ} \cdot 0$. The data illustrate a phenomenon common in protein adsorption, namely, that the smaller the difference between the $\mathrm{pH}$ and the isoelectric point of the protein, the greater the adsorption rate and the amount adsorbed (e.g. Elgersma et al. 1992; Johnson \& Matijević, 1992; Ramsden \& Prenosil, I 993).

Ellipsometry suffers from a number of difficulties of an instrumental nature connected inter alia with the passage of the incident and reflected beams through windows of the sample cell containing the solution in contact with the adsorbing substrate. These windows must be scrupulously clean to avoid introducing artefacts into the measurements of $\psi$ and $\Delta$, and it may be necessary to apply corrections for any changes of polarization of the light as it passes through them. Further difficulties concern the computation of $d_{\mathrm{A}}$ and $n_{\mathrm{A}}$ from $\psi$ and $\Delta$. For the profile depicted in Fig. 2, simple formulae exist but they cannot be solved in closed form. A typical procedure is to calculate $\psi, \Delta$ curves for a series of assumed $n_{\mathrm{A}}$ and compare the results with the experimental data. For more complicated profiles Maxwell's equations can be solved numerically in the presence of a given index profile and the calculated $\psi$ and $\Delta$ compared with the measured values (Law $\&$ Beaglehole, $198 \mathrm{I}$ ). Agreement between the calculated and measured values does not of course guarantee that the chosen model is correct. Charmet $\&$ de Gennes (1983) have shown how a refractive index profile (or rather, its Fourier transform) can be calculated directly by measuring $\psi$ and $\Delta$ at different angles of incidence, although this approach does not yet appear to have been attempted experimentally.

Most of the optical techniques require transparent materials as the adsorbing 


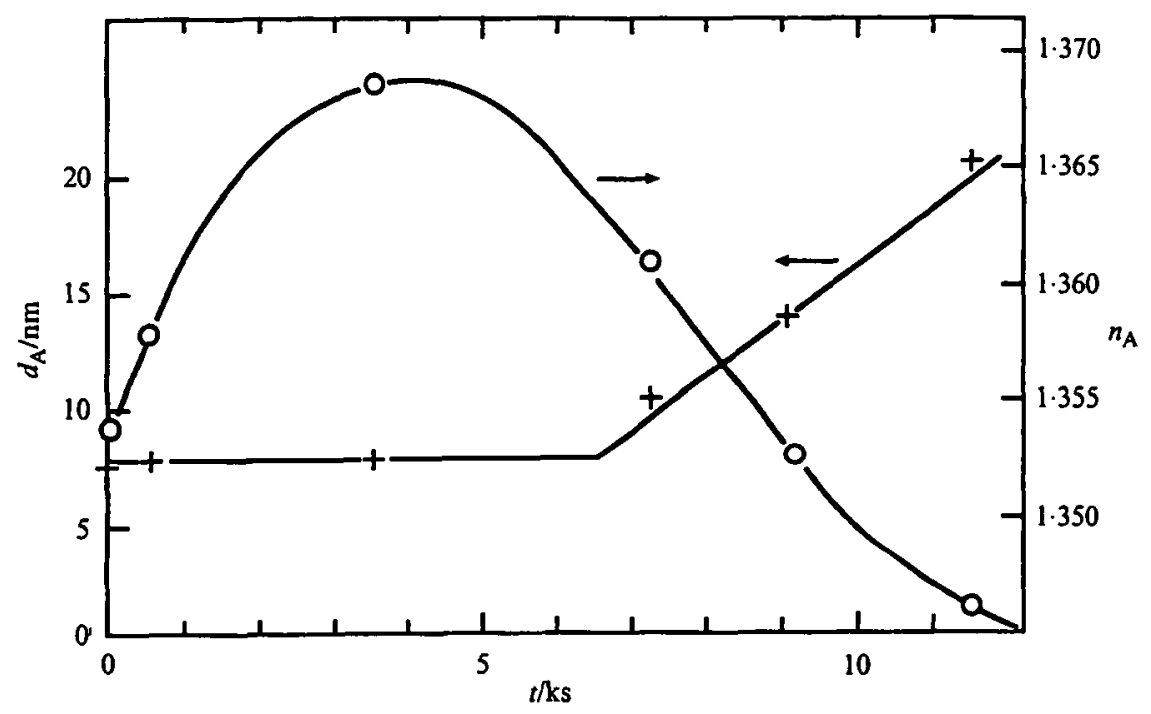

Fig. I4. Variation of $n_{\mathrm{A}}(\mathrm{O})$ and $d_{\mathrm{A}}(+)$ of fibrinogen adsorbed onto $\mathrm{SiO}_{2}$ from a solution $\left(c_{\mathrm{b}}=0.5 \mathrm{mg} / \mathrm{cm}^{3}\right)$ in $50 \mathrm{~mm}$-tris- $\mathrm{HCl}$ buffer, $\mathrm{pH} 7.35$, plus $150 \mathrm{~mm}-\mathrm{NaCl}$, determined using SAR (Schaaf \& Dejardin, 1988). Note that when the data are used to calculate $\Gamma$ using equation (57), a plateau is reached after $c a .60 \mathrm{~s}$. The observed changes in $n_{\mathrm{A}}$ and $d_{\mathrm{A}}$ therefore correspond to conformational rearrangements.

surface, but reflexion ellipsometry can be used with metallic substrates, which allows adsorption to be carried out in the presence of an applied electrical potential (Morrissey et al. 1976). Care must be taken in interpreting the results because the adsorption of small ions present in the solution, which will affect the optical constants of the interface (Paik et al. 1970), will also be modulated by the applied electric field.

\subsection{Scanning angle reflectometry $(S A R)$}

This method, developed recently by Schaaf and colleagues (1985, 1987 $a, b, 1988)$ involves measuring the reflexion coefficients $\mathscr{R}_{p}$ of a $p$-polarized beam at angles of incidence in the vicinity of the Brewster angle $\theta_{B}=\arctan \left(n_{\mathrm{F}} / n_{\mathrm{C}}\right)$. A computed $\mathscr{R}_{p}(\theta)$ curve using equation $(60)$ is then fitted to the measured points by minimizing $\Sigma_{\theta}\left(\mathscr{R}_{p, \text { calc }}-\mathscr{R}_{p \text {,expt }}\right)^{2}$, with $d_{\mathrm{A}}$ and $n_{\mathrm{A}}$ as adjustable parameters, from whose values $\Gamma$ is computed using equation (57).

The instrumentation is similar to that of ellipsometry, except that typically the incident beam is applied to the side of the interface away from the solution, which simplifies the cell but limits substrates to transparent materials. Typically a dozen or more $\mathscr{R}_{p}$ values are measured at different angles of incidence.

The reflectivity of a clean surface is zero for $p$-polarized light, as may be verified by substituting $\theta_{B}$ into equation (48), but this is no longer so in the presence of a thin adlayer. Schaaf et $a l$. $(1987 b)$ have shown that, approximately,

$\mathscr{R}_{p}\left(\theta_{B}\right) \propto \Gamma^{2}$,

which is a useful approximation if it is desired to measure rapid kinetics. 


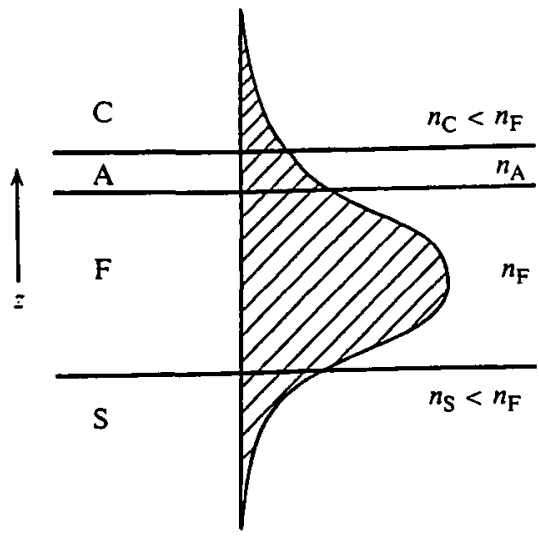

$E$ or $H \longrightarrow$

(a)

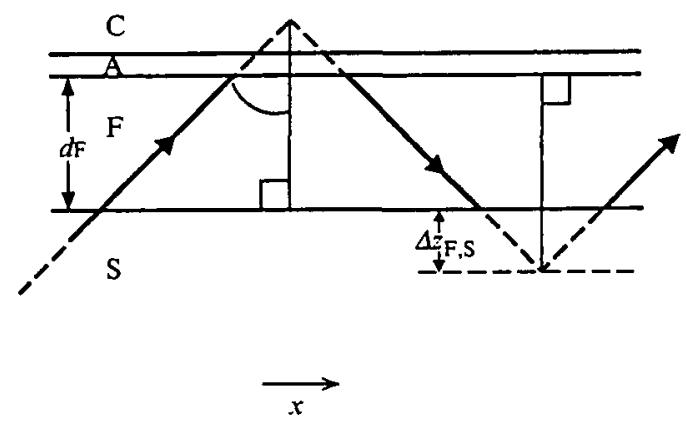

(b)

Fig. I5. (a) Electric or magnetic field distribution of the zeroth mode ( $m=0$ ) of a guided wave. (b) Ray-optic representation of a guided wave propagating in the thin film $F$, showing the various quantities referred to in the text. The layers are conventionally denoted $S$ (support), F (film), A (adlayer), and C (cover). The support is needed to give the thin film $F$ adequate mechanical strength.

The method can readily be extended to characterize diffuse refractive index profiles, and is especially useful where the thickness exceeds $\lambda / 20$ (Schaaf $e t$ al. I 985 ), when by measuring both $\mathscr{R}_{p}(\theta)$ and $\mathscr{R}_{s}(\theta)$ it may be possible to map out the complete profile.

Figure I4 shows an example of $n_{\mathrm{A}}$ and $d_{\mathrm{A}}$ obtained from reflectometry data.

\subsection{Integrated optics (IO)}

Light may be confined between two parallel totally internally reflecting interfaces, analogously to the quantum mechanical particle in a box. The walls are of finite height and thickness, hence the field is a standing wave within the box and evanescent beyond the walls, dying away exponentially (Fig. I $5 a$ ). Only certain discrete modes can exist within the box. Their eigenvalues are characterized by the propagation constant $\mathscr{B}$ ( $\equiv$ the effective refractive index $N$ (equation 50 )). The relationship between the opto-geometrical parameters of the waveguide can be derived from Maxwell's equations (Tien, I977; Ghatak \& Thyagarajan, 1989; Ramsden, I $993 \mathrm{c}$ ), but it is more convenient to obtain them from the ray-optic representation of the guided mode (Fig. $\mathrm{I}_{5} b$ ): the condition for propagation is phase invariance after each round trip comprising reflexions at the $\mathrm{F}, \mathrm{C}$ and $\mathrm{F}, \mathrm{S}$ interfaces and the double traverse of the layer $F$ (the waveguiding film). Denoting the phase shifts upon reflexion by $\Phi$, this gives us the condition:

$\Phi_{F, A, C}+\Phi_{F, S}+2 \beta_{F}=2 \pi m, \quad m=0,1, \ldots$ 


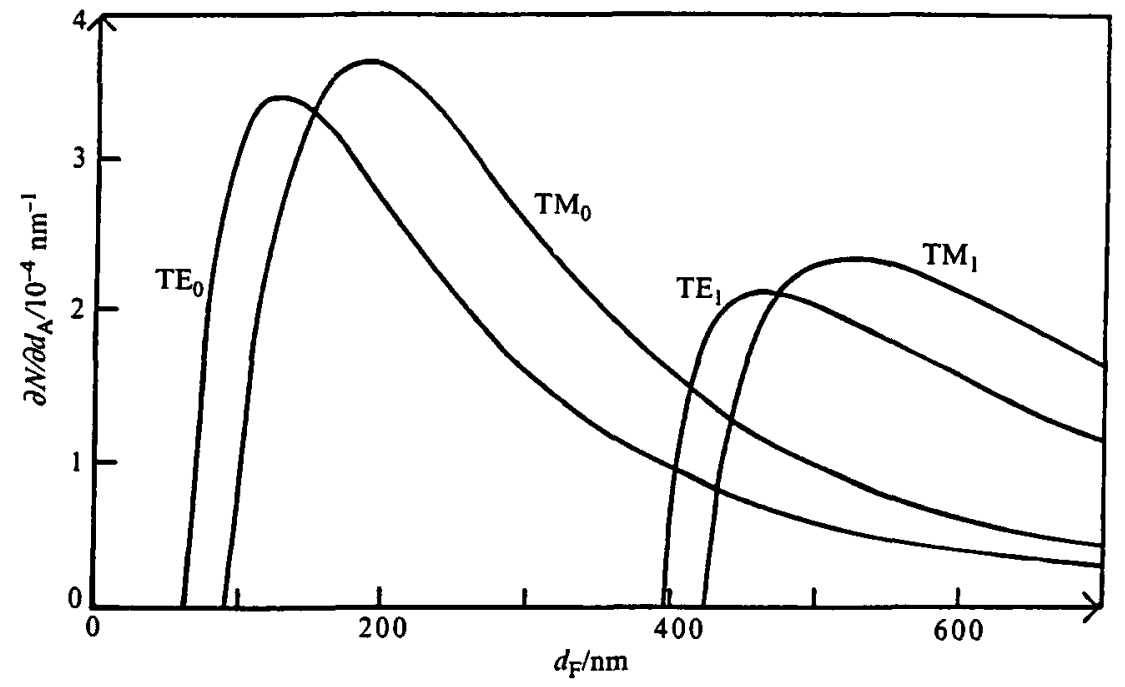

Fig. 16. Plot of the sensitivity $\partial N / \partial d_{\mathrm{A}}$ of the first two TE and TM waveguide modes as a function of waveguide thickness $d_{\mathrm{F}}$ (from Tiefenthaler \& Lukosz, 1989).

Substituting the $\Phi$ derived previously from $\mathscr{R}$ (equations $53-55,60$ ) leads to the 4-layer mode equations. For $d_{\mathrm{A}} \ll k$ (as will generally be the case in protein deposition experiments), the equations may be linearized (Tiefenthaler $\&$ Lukosz, 1989) to give :

$$
\begin{aligned}
\pi m= & k \sqrt{ }\left(n_{\mathrm{F}}^{2}-N^{2}\right)\left(d_{\mathrm{F}}+d_{\mathrm{A}} \frac{n_{\mathrm{A}}^{2}-n_{\mathrm{C}}^{2}}{n_{\mathrm{F}}^{2}-n_{\mathrm{C}}^{2}}\right)\left[\frac{\left(N / n_{\mathrm{C}}\right)^{2}+\left(N / n_{\mathrm{A}}\right)^{2}-\mathrm{I}}{\left(N / n_{\mathrm{C}}\right)^{2}+\left(N / n_{\mathrm{F}}\right)^{2}-\mathrm{I}}\right]^{\rho} \\
& -\arctan \left(\left[\frac{n_{\mathrm{F}}}{n_{\mathrm{S}}}\right]^{2 \rho} /\left[\frac{N^{2}-n_{\mathrm{S}}^{2}}{n_{\mathrm{F}}^{2}-N^{2}}\right]\right)-\arctan \left(\left[\frac{n_{\mathrm{F}}}{n_{\mathrm{C}}}\right]^{2 \rho} /\left[\frac{N^{2}-n_{\mathrm{C}}^{2}}{n_{\mathrm{F}}^{2}-N^{2}}\right]\right),
\end{aligned}
$$

where, as before, $\rho=0$ and $\mathrm{I}$ for the TE and TM modes respectively. ${ }^{17}$ Since for a layer of protein it is enough to determine the two unknowns $d_{\mathrm{A}}$ and $n_{\mathrm{A}}$; hence it is enough to measure one TE mode and one TM mode. $d_{A}$ is the same for both modes, and for isotropic layers $n_{\mathrm{A}}$ is also the same; the mode equation (73) can then be solved simultaneously and analytically to yield $n_{\mathrm{A}}$ and $d_{\mathrm{A}}$. It is then advantageous to use a thin waveguide which will only support one TE and one TM mode, because the sensitivity coefficients $\partial N / \partial d_{\mathrm{A}}$ and $\partial N / \partial n_{\mathrm{A}}$ (Fig. 16) are then optimal (Tiefenthaler \& Lukosz, 1989). If more elaborate adlayers are to be characterized (anisotropic or composed of distinguishable multilayers), then thicker waveguides supporting more modes need to be used together with correspondingly more elaborate equations, i.e. with the phase shifts in equation (72) rederived using equations $(53-55,6 \mathrm{I})$.

A waveguide is not itself an integrated-optical device, and in order to determine the effective refractive indices $N$ some way of coupling light from an external beam into the waveguide is needed (Tien, 1977). For this, a grating coupler is very

${ }_{17} \mathrm{TE}$ waves contain the field components $E_{y}, H_{x}$ and $H_{z}$ (i.e. only the electric field has a transverse component), and TM waves the components $H_{y}, E_{x}$ and $E_{z}$. They thus correspond to the $s$ and $p$ polarized plane waves discussed previously. 
$\mathrm{C}$

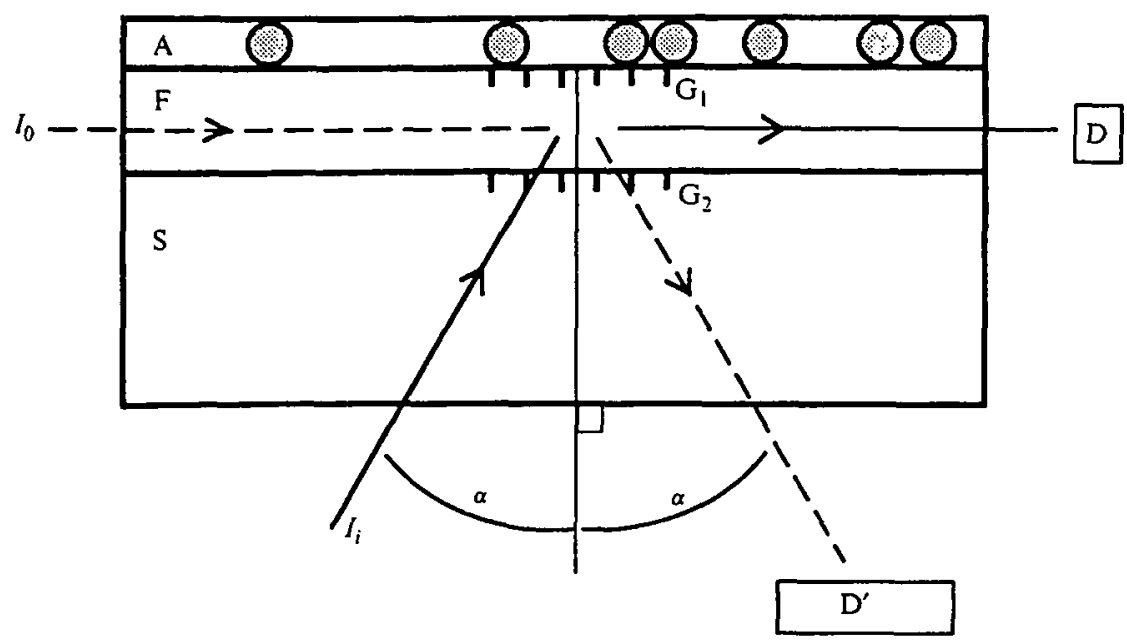

Fig. 17. Scheme of an integrated optical chip incorporating a grating either at the F, C interface $\left(G_{1}\right)$ or the $F, S$ interface $\left(G_{2}\right)$. Power coupled into the waveguide from incident beam $I_{i}$ is measured with detector $\mathrm{D}$. Power coupled out of the waveguide from incident beam $I_{0}$ is measured with detector $\mathrm{D}^{\prime}$. For protein deposition experiments, the $F, C$ interface forms one wall of a cuvette into which the protein solutions are introduced. In surface plasmon resonance (SPR), the incident beam is $I_{1}$ and the detector is $\mathrm{D}^{\prime}$. In this case $\mathrm{F}$ is a thin metal layer.

convenient, especially since the problem of fabricating low-cost, high resolution gratings was solved (Herrmann \& Wildmann, r $98_{3}$; Lukosz \& Tiefenthaler, $198_{3}$; Heuberger \& Lukosz, 1986). The grating is an integral part of the waveguide, to which it allows unencumbered access by proteins. It can be situated at either the $F, S$ or $F, C$ interface. Since the light only needs to propagate a relatively short distance (typically $\mathbf{I}-2 \mathrm{~cm}$ ) along the waveguide to the detectors, the weak coupling régime, with an incoupling efficiency of less than $10 \%$, is adequate, implying slight perturbation of the planarity of the waveguide-solution interface by the grating, if it is located at the F, C boundary. At any given angle of incidence $\alpha$ of an external beam (wave vector $k$ ), the grating generates spatial harmonics, whose components in the $x$-direction are given by $k n \sin \alpha+2 \pi l \lambda / \Lambda$, where $l=$ $\pm 1,2, \ldots$ is the diffraction order. One of them may match a possible guided mode characterized by $N$ (equation $5^{\circ}$ ), in which case we have the incoupling equation (Tiefenthaler \& Lukosz, 1989):

$N=n_{\text {air }} \sin \alpha+l \lambda / A$.

If the angle of incidence is varied while measuring the power transmitted from the grating along the waveguide to its end, where a photodetector is situated (Fig. 17), sharp resonances are observed, corresponding to the different modes and diffraction orders. The angles $\alpha$, which are measurable to micro-radian precision (Tiefenthaler, I 992), corresponding to these maxima are used to determine $N$ for the required modes using equation (74). Alternatively, light can be introduced into 


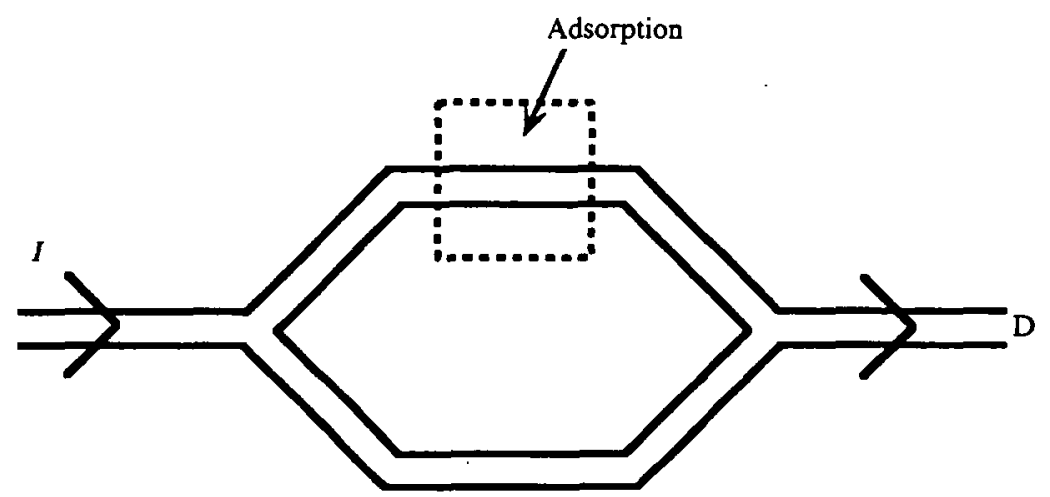

Fig. 18. Integrated-optical interferometer (Mach-Zehnder configuration).

the waveguide at one end and the grating used to couple the light out, the outcoupling angle $\alpha$ now being used to determine $N$ (Lukosz et al. 1990). Examples of data obtained with the IO technique have already been given (Fig. I), and further examples are given in Figs 22 and 23.

Instead of using in- or outcoupling as the basis for the accurate determination of $N$, interferometric methods may also be used. In the Mach-Zehnder configuration (Fig. 18), which is the integrated-optical version of the Rayleigh interferometer, a beam splitter divides the light into two arms, one of which passes through the adsorption region, and the two are then recombined (Ghatak \& Thyagarajan, 1989). In another configuration, which requires no microstructuring of the waveguides, the phase difference between TE and TM modes is measured (Lukosz \& Stamm, 1991).

\subsection{Total internal reflexion spectroscopy (TIRS)}

Again, the active element is the interface between two media of different refractive indices, such that at a suitable angle of incidence total internal reflexion occurs. Instead of measuring the change of polarization (ellipsometry or reflectometry) or the phase shift of a polarized beam (integrated-optics), the amplitude losses due to absorption by the molecular vibrations of the adsorbed molecule are measured (Harrick, 1960, I967; Fahrenfort, 1961). Since the adsorption due to an adsorbed monolayer after a single reflexion at the adsorption interface would be immeasurably small, the optical arrangement must ensure that thousands of reflexions occur, as in an optical waveguide (\$6.3), an approach taken by Swalen et al. (1978). The method is particularly valuable in the infrared region, for then it permits detailed information on the orientation, bonding state etc. of specific groups. Morrissey \& Stromberg (1974) used the technique to determine the number of carbonyl attachments per adsorbed molecule. Due account of distortions in the spectra of the thin film compared with the same material in the bulk must be taken (Yen \& Wong, 1989). The extinction at an absorbed wavelength will increase with path length and adsorbed amount, but not necessarily linearly (Bouguer-Lambert-Beer Law), nor are the absorption cross 


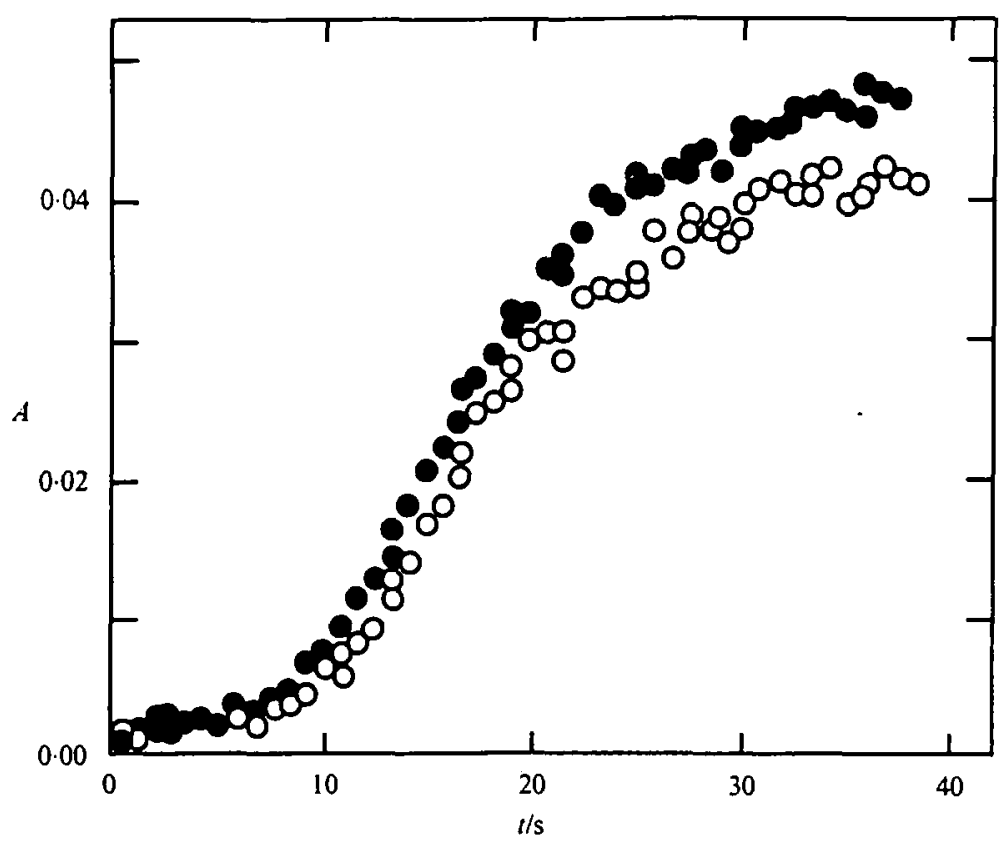

Fig. 19. Change of absorbance at $1550 \mathrm{~cm}^{-1}$ at a germanium crystal surface in contact with a solution of human serum albumin $\left(30 \mathrm{mg} / \mathrm{cm}^{3}\right)$ in $0.162 \mathrm{M}-\mathrm{NaCl}$ solution at $\mathrm{pH} 7 \cdot 4$. Absorbance was measured with a Fourier transform infrared spectrometer using attenuated total reflexion (Chittur et al. I986). Initially protein solution was introduced into the cell mounted on a clean germanium surface ( $)$. Both bulk and adsorbed protein contribute to the measured absorbance. Subsequently, the cell was flushed with pure saline, leading to a decrease of the absorbance to a new plateau, and protein solution was reintroduced $(O)$. Assuming that the surface is saturated with irreversibly bound protein during the first stage, the difference between the signals is due to protein absorbing at the interface. Solutions were flowing at a rate of $0.25 \mathrm{~cm}^{3} / \mathrm{s}$ through the cell of cross sectional area $6.4 \mathrm{~mm}^{2}$ (wall shear rate $=330 \mathrm{~s}^{-1}$ ).

sections in general known for the adsorbed species. The value of the technique therefore lies principally when it is used in conjunction with other methods used to determine $\Gamma$; TIRS then can be used to identify the adsorbed species, and deduce their conformation (although the assignment of bands may be a non-trivial task (Yen \& Wong, 1989), especially for large and complicated molecules such as proteins). ${ }^{18}$ The semiquantitative application of TIRS to obtain $\Gamma$ has been attempted by Chittur et al. (1986) in order to identify the relative numbers of individual components of adsorbed mixtures of proteins. These authors used a Beer expression:

$\left(\log I_{i} / I_{t}\right)_{\lambda}=c_{j} K_{j, \lambda}$,

where the $K_{j, \lambda}$ were obtained from the spectra of the individual components and of stranded mixtures, calibrated using radiolabelling. Fig. I 9 shows some of their results. The signal due to the adsorbed material is due to the difference between the two sets of data.

Ix 'Total internal reflexion resonance Raman spectroscopy, which would offer significant advantages in the assignment of protein vibrations (Spiro, 1985), does not appear to have been attempted. 


\subsection{Surface plasmon resonance (SPR)}

Non-radiative propagating charge density waves can exist at the surface of a metal characterized by a complex permittivity $\hat{\epsilon}_{\mathrm{M}}=\epsilon_{\mathrm{M}}^{\prime}+i \epsilon_{\mathrm{M}}^{\prime \prime}$ provided that the conditions

$\epsilon_{M}^{\prime}<-1$

and

$\left|\epsilon_{\mathrm{M}}^{\prime}\right|>\epsilon_{\mathrm{C}}=n_{\mathrm{C}}^{2}$

are fulfilled (Kretschmann, 1971; Agranovich \& Mills, 1982; Lukosz, 1991). These surface plasmons or polaritons are $p$-polarized electromagnetic waves propagating parallel to a metal-dielectric interface with wave vector $k_{M, x}$ given by:

$k_{\mathrm{M}, x}=k \sqrt{ }\left[\varepsilon_{\mathrm{C}} \hat{\epsilon}_{\mathrm{M}} /\left(\varepsilon_{\mathrm{C}}+\hat{\epsilon}_{\mathrm{M}}\right)\right]$.

Perpendicular to the interface the wave is evanescent and decays as $\exp \left(z / \Delta z_{\mathrm{M}, \mathrm{C}}\right)$, where

$\Delta z_{\mathrm{M}, \mathrm{C}}=\sqrt{ }\left(-\epsilon_{\mathrm{M}}^{\prime}\right) /\left(k n_{\mathrm{C}} N\right)$;

here an effective index $N$ is defined in analogy with the guided modes in an optical waveguide $(\$ 6.3)$ by (Lukosz, 1991):

$N=\mathrm{I} / \sqrt{ }\left(\mathrm{I} / n_{\mathrm{C}}^{2}+\mathrm{I} / \epsilon_{\mathrm{M}}^{\prime}\right)$.

A thin film characterized by $d_{\mathrm{A}}(\ll \lambda)$ and dielectric constant $\hat{\epsilon}_{\mathrm{A}}$ results in a shift $\Delta k_{\mathrm{M}, x}$ Kretschmann, $197 \mathrm{I}$; Kolb, 1982) of the wave vector:

$\Delta k_{\mathrm{M}, x}=k_{\mathrm{M}, x}^{2} \frac{\sqrt{ }\left(-\epsilon_{\mathrm{C}} \hat{\epsilon}_{\mathrm{M}}\right)}{\epsilon_{\mathrm{C}}-\hat{\epsilon}_{\mathrm{M}}}\left(\mathrm{I}-N\left(\frac{\mathrm{I}}{\hat{\epsilon}_{\mathrm{A}}}+\frac{\hat{\epsilon}_{\mathrm{A}}}{\epsilon_{\mathrm{C}} \hat{\epsilon}_{\mathrm{M}}}\right)\right) d_{\mathrm{A}}$,

where, for the non-absorbing films we are considering, $\hat{\epsilon}_{\mathrm{A}}=n_{\mathrm{A}}^{2}$. Unlike guided modes in an optical waveguide, surface plasmons are highly attenuated as they propagate parallel to the interface, decaying as

$\Delta x_{\mathrm{M}}=\left(\epsilon_{\mathrm{M}}^{\prime}\right)^{2} /\left(k \epsilon_{\mathrm{M}}^{\prime \prime} N^{3}\right)$;

typically $\Delta x_{\mathrm{M}}$ is only a few $\mu \mathrm{m}$, which limits the area available for measuring surface phenomena.

A typical experimental arrangement (cf. Fig. 17) is to evaporate a thin film of a nobel metal ( $\mathrm{Au}, \mathrm{Ag}, \mathrm{Pt}$ etc. in order to avoid the formation of a tarnishing film) on the surface of a hemicylindrical prism (Kretschmann, I 97I) or a diffraction grating (Cullen et al. 1987/88). The intensity of the reflected beam is monitored as a function of the angle of incidence onto the prism or the grating. In the case of the prism (refractive index $n_{\mathrm{S}}$ ), with $\alpha$ as the angle of incidence onto the interface, coupling is achieved when the condition:

$k_{\mathrm{M}, x}=k n_{\mathrm{S}} \sin \alpha$

is fulfilled (Kretschmann, I $97 \mathrm{I}$ ), whereupon a sharp dip in the reflected intensity 
is observed. Calling this angle $\alpha_{\min }, \Delta k_{\mathrm{M}, x}$ due to protein adsorption can be determined from the shift in $\alpha_{\mathrm{min}}$, and if either $d_{\mathrm{A}}$ or $n_{\mathrm{A}}$ are already known the complementary unknown parameter may be found using equation $(8 \mathrm{I})$. The optical constants of the metal can be obtained from measurements of $k_{\mathrm{M}, x}$ for the clean surface prior to initiating adsorption using equation (78), for which purpose SPR was indeed first used (Kretschmann, 1971 ). Both $d_{\mathrm{A}}$ and $n_{\mathrm{A}}$ can be obtained by fitting equation $(8 \mathrm{I})$ to the complete dispersion relation mapped out by varying $\alpha$ or $\lambda$, a time-consuming procedure similar to that discussed for SAR in $\S 6.2$, and one which precludes the measurement of fast kinetics.

\subsection{Evanescent wave light scattering ( $E W L S)$}

In this rather new method, which has only just been realized experimentally at the air-water interface (Lin \& Rice, I 993), the intensity of scattered evanescent light at different angles in the plane parallel to the interface is measured, and fitted to the structure function for hard disks at the surface. Since, at the time of writing, only one brief communication on EWLS has appeared, it is too early to elaborate, but the technique appears likely to fill an important gap, namely the in situ, realtime determination of the lateral distribution of molecules.

\section{LABELLING TECHNIQUES}

This section covers a diverse collection of methods in which some attribute of the molecule under investigation is enhanced to make its detection easier. Labelling can be applied to some of the methods already discussed; for example, by radioactively labelling molecules in the solution depletion techniques, smaller concentrations than would otherwise be possible can be detected. Heavy or highly polarizable moieties can be covalently attached to proteins to enhance the response of the gravimetric and optical methods respectively. The general disadvantage of the labelling procedure is that the properties of the protein, especially those relevant to transport and adhesion, are likely to be significantly altered by the addition of such groups. A further disadvantage is the extra work involved in synthesizing and purifying the labelled protein. Moreover, during lengthy kinetic runs there is also the danger that the label could become detached. Therefore, labelling should preferably only be contemplated in special circumstances in order to yield information which cannot otherwise be obtained. Such a circumstance arises when it is important to identify which particular species is adsorbed among several candidates. With the exception of TIRS, the methods described up until now are not qualitatively sensitive to the type of molecule adsorbed. ${ }^{19}$ If the molecules are labelled, however, the surface excess of each can be probed directly. In this way the important phenomenon of protein exchange, whereby an 'irreversibly' adsorbed protein can be displaced by another one, was demonstrated using radiolabelled congeners (Lutanie et al. 1992).

\footnotetext{
19 I.e. the measured signal only differs quantitatively from one species to another, hence when two or more species are present together, their relative presence in the adsorbed adlayer can only be inferred indirectly from a kinetic analysis.
} 


\subsection{Radiolabelling}

If proteins are labelled radioactively by enrichment in ${ }^{3} \mathrm{H}$ or ${ }^{13} \mathrm{C}$, perturbation to the molecule is minimal; attachment of ${ }^{125} \mathrm{I}$ is the method of choice, however, because it is chemically much easier ${ }^{20}$ and the specific radioactivity is higher. The labelled molecule is allowed to adsorb on the substrate, which may be chosen without regard to geometry and material type. After a certain time, the protein solution is removed and the radioactivity due to proteins attached to the surface measured (van Dulm \& Norde, 1983; Nieto et al. 1986). The well-known immutability of radioactivity ${ }^{21}$ allows drastic methods to be employed to comminute the substrate and introduce it into the counting device, and permits the independence of the radioactive count rate from the environment of the labelled protein to be assumed. A high precision of the count rate can be attained by rigorous shielding and counting over long periods. It is therefore one of the most reliable methods for determining adsorption. Apart from the need to label the protein, which is shared by all the methods discussed in this section, the only two disadvantages are that the presence of the label may alter the hydrodynamic and adsorption properties of the protein (Soderquist \& Walton, 1980), ${ }^{22}$ and that the method is off-line; hence kinetics can only be obtained by the laborious assembly of results from substrates exposed to the protein solution for different lengths of time. Since the preparation of reproducible substrates is one of the most difficult features in connexion with adsorption work, this is a non-trivial difficulty. Hence, radioactive labelling is employed only in special cases, such as when unusual (non-planar) substrate geometries, incompatible with other methods, are used, and to study protein exchange reactions, which require the use of at least two distinguishable populations of molecules. Fig. 20 shows an example of data acquired using radiolabelling.

\subsection{Enzyme-linked immunoassay (ELISA)}

In this technique, introduced by Engvall \& Perlmann (1 97 I), proteins are labelled by covalently attaching an enzyme molecule to them. After exposure of the adsorbent surface to the protein solution, the surface is washed and exposed to any substrate of the enzyme, selected such that the product is easily detected (e.g. by virtue of a colour change). Signal amplification is therefore proportional to the turnover number of the enzyme and the length of time to which it is exposed to its substrate. Although the perturbation of a protein molecule by an attached enzyme is probably too great to allow this technique to be used to investigate protein adsorption, it may be of potential application if the protein of interest is itself an enzyme. The procedure suffers from the same disadvantage as the radiolabelling method, i.e. it is intermittent (off-line).

20 Well-established protocols exist.

21 But see the interesting observation of Gray \& Ramsay (1909).

22 In the literature, one finds statements that the labelling does affect these properties, as well as assertions to the contrary. The investigator would be well advised to check this point for the particular system under scrutiny. 


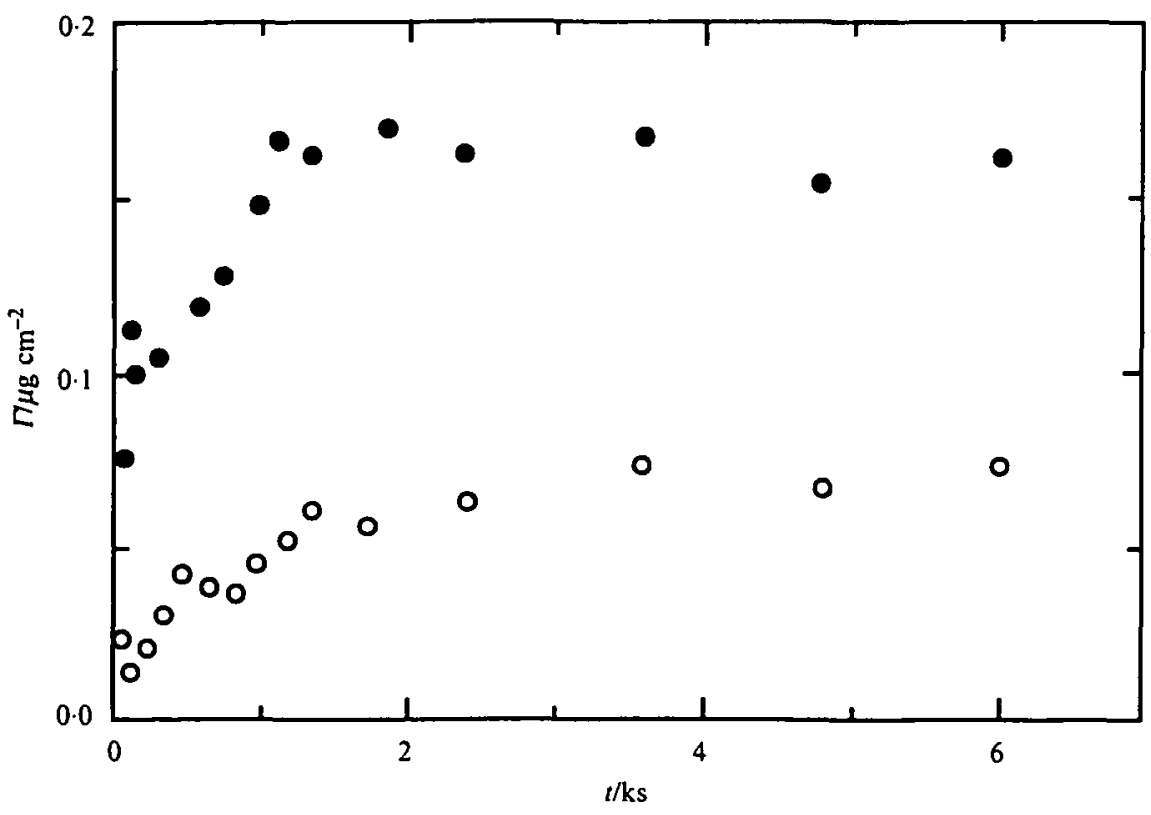

Fig. 20. Kinetics of adsorption of human serum albumin labelled with ${ }^{125} \mathrm{I}$ from an agitated solution (initial concentration $\left.=40 \mu \mathrm{g} / \mathrm{cm}^{3}\right)$ in tris- $\mathrm{HCl}$ buffer $(25 \mathrm{mM}, \mathrm{pH} 7.4)$ onto glass (O) and silanized glass (O), at $T=22{ }^{\circ} \mathrm{C}$. After incubation for the desired period, the adsorbed protein was transferred to a $\gamma$-ray counting tube, calibrated using known quantities of labelled albumin solution (van Dulm \& Norde, r 983 ).

Vast effort has been expended on developing the technique into an immunoassay for the diagnosis of infectious diseases (Wreghitt \& MorganCapner, 1990; Porstmann \& Kiessig, 1992). At the present level of this application, it is sufficient for the adsorption of the relevant protein (often an antibody) to be qualitatively determined. Severe problems attend its development into a quantitative technique. The yield of enzyme-catalyzed substrate transformation depends greatly on hydrodynamic factors, which govern not only the transport of substrate to the enzyme, but also the removal of products; it must be borne in mind that many enzymes are inhibited by product accumulation. Hydrodynamic conditions in an ELISA experiment are, as a rule, extremely illdefined. The solutions may be agitated by shaking, which produces irregular currents practically impossible to quantify. A totally stationary solution is amenable to an exact hydrodynamic treatment, but exacerbates the inherent nonlinearity in the measured response (amount of transformed substrate) to the duration of the exposure of the adsorbed enzyme to its substrate, to the substrate concentration, and to the surface concentration of enzyme. The turnover number of the enzyme at the surface may differ from that measured in homogeneous solution, because of conformational restraint. The degree of restraint will depend on the orientation of the protein, which will also control access of the substrate to the active site of the molecule. Furthermore, enzyme activity is usually sensitive to $\mathrm{pH}$ and salt concentration, the surface values of which usually differ from the 
bulk values ( $\$ 5$, equation 30 ). No examples of this technique applied to the quantitative determination of protein absorption kinetics have been reported in the literature.

\subsection{Total internal reflexion fuorescence microscopy (TIRFM)}

This method is the most sophisticated of the labelling techniques, being the only one capable of continuous on-line measurement, and hence the determination of kinetic curves. Fig. 12 can be used to visualize the experimental requirements. ${ }^{23}$ A beam of light of a wavelength suitable for exciting photoluminescence of the molecule under study ${ }^{24}$ is directed through the adsorbing substrate, which must be transparent and have a higher refractive index than the medium in which the molecule under investigation is dissolved or suspended, onto the interface, at an angle sufficiently large to ensure that total internal reflexion takes place. As we have seen in $\S 6$, under these conditions the light undergoes a short excursion into the rarer medium before returning. The evanescent field in the rarer medium excites photoluminescence of the molecule. Since the evanescent field decays exponentially into the solution, molecules in the vicinity of the interface are selectively excited. The fluorescent emission is collected by microscope optics focused onto the interface. The measured fluorescence intensity $I_{f}$ depends in a complicated way on the exponentially decaying evanescent exciting field, the emitted light, the microscope collection optics, and the concentration profile of the fluorophore adjacent to the interface:

$I_{f}=I_{i} K_{f} \int_{0}^{\infty} c(z) \phi(c) \exp \left(-z / \Delta z_{\mathrm{F}, \mathrm{C}}^{\prime}\right) \mathrm{d} z$,

where $K_{f}$ gives the collection efficiency. $\phi$ is the fluorescence quantum yield which may diminish in the adsorbed layer due to concentration-dependent selfquenching, and $\Delta z_{\mathrm{F}, \mathrm{C}}^{\prime}$ takes into account the attenuation of the evanescent wave due to adsorption by the fluorophores; if the absorption is small, then (Rondelez et al. (987):

$\Delta z_{\mathrm{F}, \mathrm{C}}^{\prime}=\Delta z_{\mathrm{F}, \mathrm{C}} a^{\prime} /\left(\Delta z_{\mathrm{F}, \mathrm{C}}+a^{-1}\right)$,

where $\Delta z_{F, C}$ is calculated as before (equations $6_{3}$ or $6_{4}$ ) and $a$ is the Lambert absorption coefficient. $\Delta z_{\mathrm{F}, \mathrm{C}}^{\prime}$ can be calculated exactly by using a complex $n_{\mathrm{C}}$ in equations $\left(6_{3}\right)$ or $\left(6_{4}\right)$.

For the concentration profile depicted in Fig. 2, the contribution of dissolved molecules to the collected fluorescence can be neglected (or, at least then merely contributes a constant background signal). If $\phi$ can be assumed to be constant, the measured intensity is then proportional to $\Gamma$.

The computation of absolute values of $\Gamma$ from $I_{f}$ poses severe problems. The factor $K_{f}$ is very difficult to calculate. An even greater difficulty is the

23 Details are well documented by Axelrod et al. (1985).

24 Sometimes the natural fluorescence of aromatic amino acids is sufficient (Iwamoto et al. 1985); otherwise a fluorescent label must be chemically attached. 
determination of $\phi$; not only may it be concentration-dependent, but also dependent on local $\mathrm{pH}$, which, as we have seen, may differ by one or more units from the bulk value $\left(\xi_{5}\right.$, equation 30 ). Moreover, both the photoluminescent excitation and emission spectra may change upon adsorption (Andrade et al. 1984; Iwamoto et al. 1985). Provided that the concentration-dependent variations are slight, however, the measured $I_{f}$ may be calibrated, e.g. using the radiolabelling technique (Kulik et al. I99I).

Although TIFRM discriminates between adsorbed and solution molecules less well than the optical methods discussed in $\$ 6$, it possesses the advantage of being able to be used for spatially imagining adsorbed proteins, enabling, for example, the distribution of molecules on substrates with a gradient of varying wettability to be determined (Hlady, I99I), and the detection of clustering of the adsorbed molecules on the surface (Kalb \& Engel, I991). In principle, by varying the angle of incidence and hence $\Delta z_{F, C}^{\prime}$, and assuming $\phi$ to be constant, the complete concentration profile at the interface can be found by carrying out the inverse Laplace transform of the $I_{f}(\theta)$ data. In practice the experimental data is not sufficiently complete or noise-free to enable this to be done, and the Laplace transform of a plausible concentration profile is computed and fitted to the experimental points (Rondelez et al. 1987). By slightly varying the measurement set up, the lateral diffusion coefficient of adsorbed proteins can be determined from the kinetics of fluorescence recovery after photobleaching (FRAP). In this method, the adsorbed proteins are exposed, through a patterned mask, to a flash of light powerful enough to irreversibly photolyze the fluorophore. The observed intensity slowly recovers by lateral diffusion of unbleached molecules from the unphotolyzed into the photolyzed regions. (Tilton et al. 1990). Provided variable wavelength excitation and emission detection are available, TIRFM can be used to observe populations with dissimilar fluorescence spectra simultaneously and independently.

\subsection{Resonance energy transfer (RET)}

In this ingenious method the substrate on which adsorption takes place must possess fluorophores with an excitation spectrum overlapping the emission spectrum of adsorbing fluorescent proteins; if the surface fluorophores are excited, luminescence at the wavelength corresponding to the protein emission maximum is detected, or vice versa. Energy is transferred from the excited donor to the acceptor by the Förster (1948) resonance mechanism, the efficiency of which depends on the inverse sixth power of the distance $r$ between energy donor and acceptor. Hence in effect only adjacent molecules (i.e. bound to the substrate) can contribute to the measured intensity. The method has been applied using fluorescently labelled antibody as acceptor and small suspended liposomes made from labelled lipids as the substrate and donor (Tamm \& Bartoldus, 1988). The rate $k_{F}$ of energy transfer is given by:

$k_{F}=\left(r_{0} / r\right)^{6} / \tau_{D}$, 
where $\tau_{D}$ is the fluorescent lifetime of the donor and the coefficient $r_{0}$ depends on the overlap between the donor emission $\left(\mathscr{E}_{D}\right)$ and acceptor excitation $\left(\mathscr{E}_{\mathrm{A}}\right)$ spectra:

$r_{0}=K_{F} \int_{0}^{\infty} \mathscr{E}_{D}(\lambda) \mathscr{E}_{\mathrm{A}}(\lambda) \lambda^{4} \mathrm{~d} \lambda$

The constant $K_{F}$ depends on many factors such as the inverse fourth power of the refractive index of the medium through which transfer occurs, the orientation of the transition dipoles of donor and acceptor, and the quantum yield of donor photoluminescence, many of which can only be estimated. The difficulties inherent in calculating $\Gamma$ from the measured acceptor emission intensity are thus even greater than in the case of TIRFM.

RET could in principle be applied to the TIRFM method to enhance its selectivity for molecules bound to the surface, though this does not yet appear to have been attempted.

\section{EVALUATION OF KINETIC PARAMETERS}

A general kinetic scheme is shown in Fig. $2 \mathrm{I}$. At a distance $d_{1}\left(d_{1} \approx d_{\mathrm{A}}\right)$ above the interface, the concentration of protein in solution is $c_{1}$. Application of the continuity principle to this very thin layer $\left(\mathrm{d} c_{1} / \mathrm{d} t=0\right)$ gives

$\mathcal{f}+\Gamma k_{d}=\left(k_{a}+k_{s}\right) c_{1} \phi$.

$\mathcal{F}$ is the flux of molecules towards the interface. It depends on the hydrodynamic regimen; for a stationary fluid in which mass transport is governed by pure diffusion (Crank, 1975),

$\mathcal{F}=\left(c_{b}-c_{1}\right) \sqrt{ }(D /(\pi t))$

whereas for a moving fluid the Nernstian expression

$\mathcal{F}=\left(c_{b}-c_{1}\right) D / W_{d}$

applies, with $W_{d}$, the thickness of the diffusion boundary layer, given by expressions depending on the flow rate, solution viscosity, rotation rate of an electrode, etc. (Levich, 1962); under typical experimental conditions $W_{d} \approx$ $100 \mu \mathrm{m} . k_{d}$ may be more complicated than a simple first-order rate constant if lateral interactions are taken into account (Kurrat et al. 1993). $k_{a}$ and $k_{\delta}$ depend on energy barriers impeding adsorption; $k_{a}$ refers to reversibly adsorbed molecules, and $k_{s}$ to irreversibly adsorbed ones. Heterogeneous surfaces characterized by a distribution of $k_{a}$ and $k_{s}$ may also have to be taken into account (Kulik et al. 1991). The presence of irreversible processes renders the usual thermodynamic (Langmuir) isotherm inapplicable to the problem.

$\phi$ denotes the fraction of the surface available for deposition. In the Langmuir formalism,

$\phi=\mathbf{I}-\theta$ 


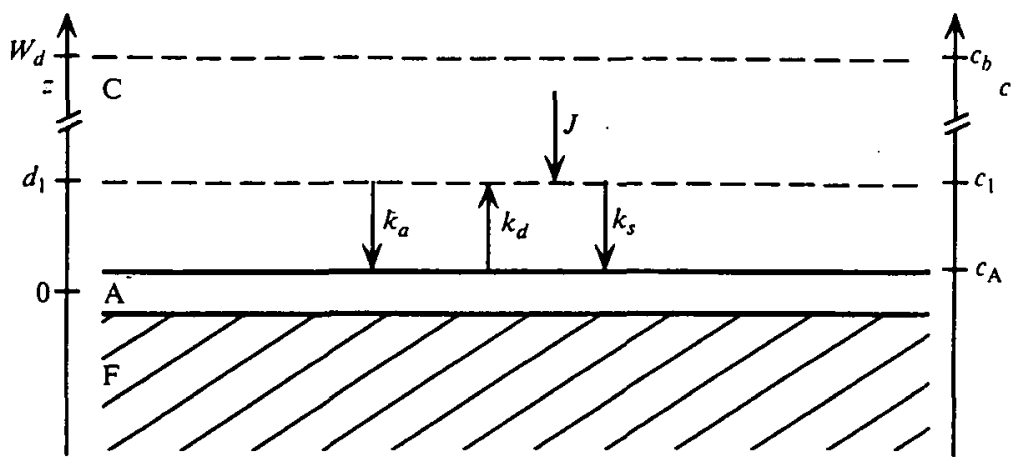

Fig. 21. Kinetic scheme for evaluating $\Gamma(t)$ data.

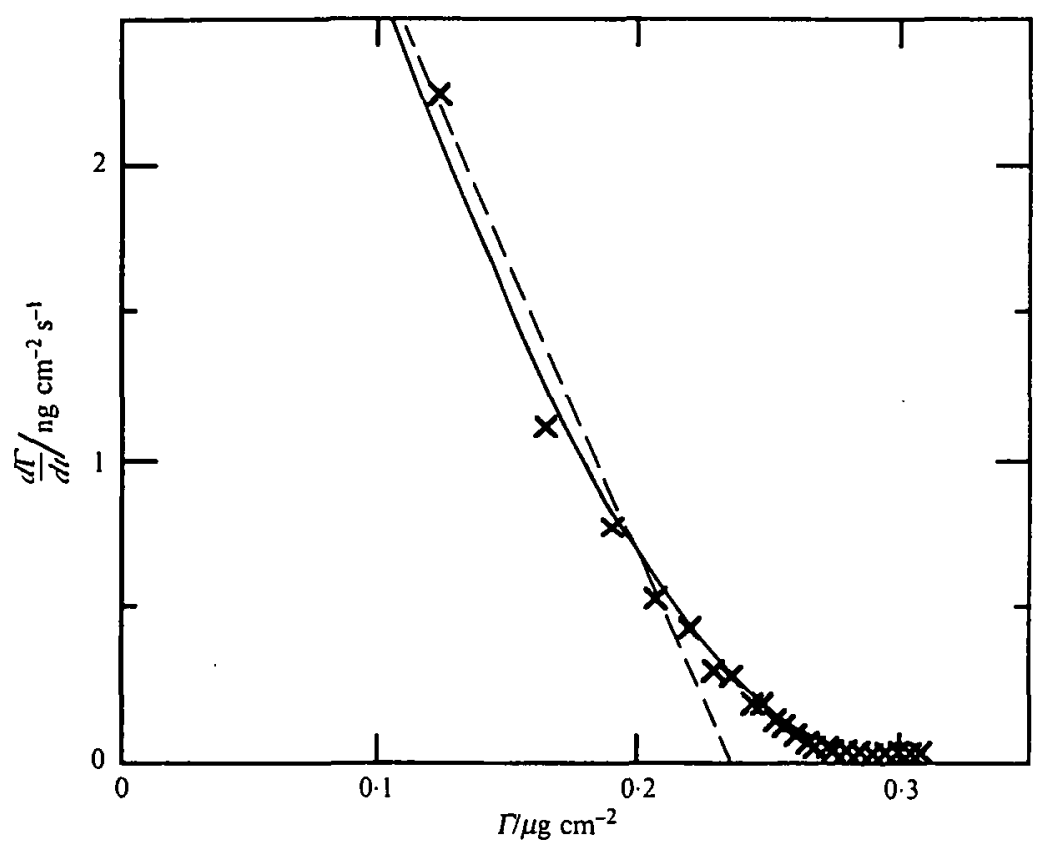

Fig. 22. Illustration of the inapplicability of Langmuir kinetics to protein adsorption (apotransferrin dissolved in $10 \mathrm{~mm}$ hepes buffer, $\mathrm{pH} 77^{\circ}$ ) onto $\mathrm{SiTiO}_{2}, T=25^{\circ} \mathrm{C}$, wall shear rate $\mathrm{I} 7 \mathrm{~s}^{-1}$ ). Continuous line: kinetic scheme (equations 88 and 90 ) with $\phi$ given by (92); dashed line, idem, except $\phi$ given by the Langmuir expression (91). $\Gamma$ determined using the IO technique (Ramsden, 1993b).

but this applies only to a surface with a definite number of adsorption sites, and then only when the sites are bigger than the ligands. It does not give the correct form of protein adsorption kinetics where the adsorbing surface is a continuum, such as a bilayer lipid membrane (Ramsden, $1993 b$; Ramsden $\&$ Prenosil, 1993). In this case an expression involving successive powers of $\theta$ must be used (Schaaf \& Talbot, 1989; Dickman et al. 1991):

$\phi=\mathrm{I}-4 \theta+3 \cdot 30797 \theta^{2}+\mathrm{I}_{4} 40688 \theta^{3}+O\left(\theta^{4}\right)$. 


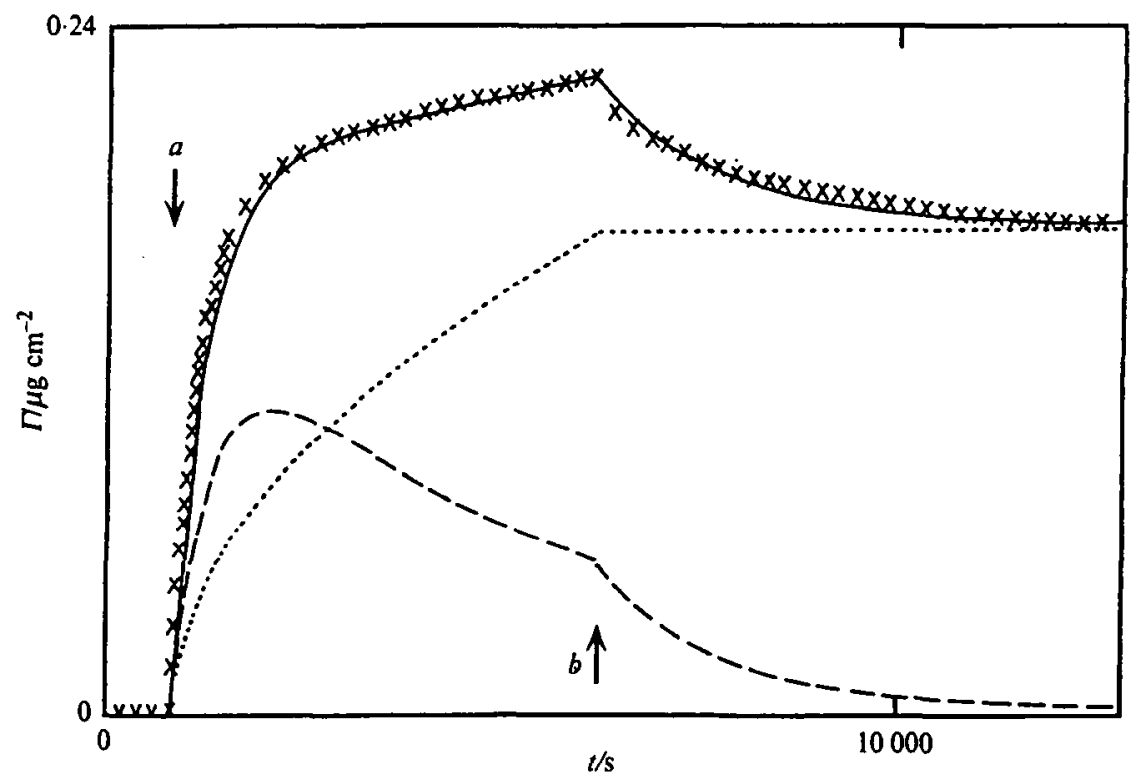

Fig. 23. The adsorption of human serum albumin dissolved in $10 \mathrm{~mm}$ hepes buffer, $\mathrm{pH} 7 \cdot 34$, $c_{\mathrm{b}}=82 \mu \mathrm{g} / \mathrm{cm}^{3}$, to a SiTiO ${ }_{2}$ surface at $T=25^{\circ} \mathrm{C}$ (wall shear rate $0.32 \mathrm{~s}^{-1}$ ). (x): $\Gamma$ determined using the IO technique (Kurrat et al. 1993). Pure buffer was flowing through the adsorption cell except between the arrows $a$ and $b$, when protein solution flowed. The solid line denotes total amount adsorbed, the dashed line the amount reversibly adsorbed, and the dotted line the amount irreversibly adsorbed, according to the kinetic scheme of Fig. $2 \mathrm{I}$.

Figure 22 clearly demonstrates the inadequacy of Langmuir kinetics (equation 91) compared with (92) to describe the adsorption of a globular protein, transferrin, onto a smooth hydrated metal oxide $\left(\mathrm{SiTiO}_{2}\right)$ surface.

In order to evaluate the kinetic parameters, the experimental data is fitted to the expression for $\Gamma$ or $\mathrm{d} \Gamma / \mathrm{d} t$ constructed according to a scheme like that in Fig. $2 \mathrm{I}$ with more or less terms depending on the particular problem. Since usually many hundreds of data points are available for a kinetic curve, fitting to three or four parameters can be accomplished rather accurately and reliably. Only in a few very simple instances can the necessary parameters be read off an appropriate plot of the data directly.

Many models for protein adsorption have been proposed (e.g. Andrade \& Hlady, r986; Lundström \& Elwing, r989; Kulik \& Sevastyanov 1991), usually incorporating steps of conformational change, etc. It now appears possible, with the availability of high-quality kinetic data, to test these models rigorously. As an example, Fig. 23 illustrates the application of the model depicted in Fig. 2 I to the adsorption of human serum albumin onto a planar $\mathrm{SiTiO}_{2}$ surface. This figure also demonstrates the importance of taking the entire kinetics into account when considering adsorption mechanisms, rather than only the initial kinetics, which has often been done in the past. 


\section{COMPARISON OF TECHNIQUES}

At the beginning of this review a number of attributes which should be possessed by 'good' techniques were mentioned. Tables $\mathrm{I}$ and 2 will help to put the degree of approach to the ideal method into perspective. Below we remark on some specific aspects.

Sensitivities of the different techniques. It would be highly desirable to be able to compare techniques on a I-dimensional continuum. Fig. 24 depicts the generalized measurement procedure: a certain quantity of adsorbed molecules $\Gamma$ gives rise to a signal $X$, which may be a velocity, a frequency change, an angle etc. $X$ may subsequently be converted to an electrical or optical signal. Clearly the coefficient $\partial X / \partial \Gamma$ should be as large as possible, but since its units depend on those of $X$, it is not useful for comparing one technique with another. Of more use is the ratio of the signal $(S)$ to the noise $(N)$. The signal is the total number of molecules adsorbed on the detector area, $(A)$, that is

$S=\Gamma A$.

Let us say that a perfect detector can detect a signal which is at least as large as one standard deviation $\left(\sigma_{S}\right)$ of consecutively measured points (a multiple of 2, 3 or more standard deviations could be taken without changing the argument). The minimum possible noise is that due to random fluctuations in the input signal. The simple adsorption process may be approximated by a Poisson distribution, for which $\sigma_{S}=N=\sqrt{ } S$. The measured signal to noise ratio may then be normalized against its maximum values, $(S / N)_{\max }$, which is $S / \sigma_{S}=S / \sqrt{ } S=$ $\checkmark S$. The normalized ratio can vary between o (for the worst possible detector, incapable of detecting a signal) and $\mathrm{I}$ (the best possible detector). The square of the normalized ratio has been called the detectivity, $\varepsilon$, in the context of radiation detectors (Jones, 1952; Zweig, 1964). Hence

$\varepsilon=\frac{(S / N)_{\text {meas }}^{2}}{(S / N)_{\max }^{2}}$

In practice, the measured noise, $N_{\text {meas }}$, may depend on factors wholly independent of $S$, such as mechanical or electrical instabilities in the measuring apparatus. In this case we have

$\varepsilon=\frac{\left(S / N_{\text {meas }}\right)^{2}}{\left(S / \sigma_{S}\right)^{2}}=\frac{\sigma_{S}^{2}}{N_{\text {meas }}^{2}}=\frac{\Gamma A}{N_{\text {meas }}^{2}}$.

Hence, other things being equal, the greater the adsorbing surface $A$ (see Table I), the greater the detectivity of the technique.

For many of the techniques discussed, however, the major contribution to the precision of the measurement of $\Gamma$ is not instrumental noise, but uncertainties in the parameters required to calculate $\Gamma$ from the measured quantity (Table 2). Only in a few cases can all the necessary parameters be determined independently. 
Table I. Summary of techniques and their applicability to various substrates

\begin{tabular}{|c|c|c|c|c|}
\hline Technique & $\begin{array}{l}\text { Typical time } \\
\text { resolution }\end{array}$ & $\begin{array}{l}\text { Typical } \\
\text { measuring area }\end{array}$ & $\begin{array}{l}\text { Substrate } \\
\text { morphology }\end{array}$ & $\begin{array}{l}\text { Substrate } \\
\text { material }\end{array}$ \\
\hline BSD & $\min -\mathrm{hr}$ & $\mathrm{m}^{2}$ & Colloid, powder & Unlimited \\
\hline FD & $s$ & $\mathrm{~m}^{2}$ & Colloid, powder & Unlimited \\
\hline $\mathrm{CSD}$ & $s$ & $\mathrm{~m}^{2}$ & Powder & Unlimited \\
\hline QCM & $\mathrm{s}$ & $\mathrm{cm}^{2}$ & Planar & Metal \\
\hline SAW & $\mathbf{s}$ & $\mathrm{cm}^{2}$ & Planar & Quartz \\
\hline \multicolumn{5}{|l|}{ Colloid } \\
\hline Diffusivity & $\min$ & $\mathrm{cm}^{2}$ & Colloid & Unlimited \\
\hline Capillary flow & $\min$ & $\mathrm{m}^{2}$ & Capillaries & Unlimited \\
\hline MIC & $\mathbf{s}$ & $\mathrm{cm}^{2}$ & Planar & Metal oxide ${ }^{b}$ \\
\hline SCI & $\mathbf{s}$ & $\mathrm{cm}^{2}$ & Planar & Metal oxide ${ }^{b}$ \\
\hline FET & $\mathbf{s}$ & $\mathrm{cm}^{2}$ & Planar & Metal oxide ${ }^{b}$ \\
\hline IFCM & s & $0.1 \mathrm{~mm}^{2}$ & Planar & Lipid membrane \\
\hline Ellipsometry & $\min$ & $\mathrm{mm}^{2}$ & Planar & Wide range \\
\hline SAR & $\min$ & $\mathrm{mm}^{2}$ & Planar & Transparent ${ }^{b}$ \\
\hline IO & $\mathbf{s}$ & $\mathrm{mm}^{2}$ & Planar & Transparent $^{b}$ \\
\hline SPR & $\mathrm{s}-\mathrm{min}$ & $\mu \mathrm{m}^{2}$ & Planar & $\begin{array}{l}n_{\mathrm{F}}>n_{\mathrm{C}} \\
\text { Metal }\end{array}$ \\
\hline TIRFM & $\mathrm{s}$ & $\mathrm{mm}^{2}$ & Planar & Transparent ${ }^{b}$ \\
\hline & & & & $n_{\mathrm{F}}>n_{\mathrm{c}}$ \\
\hline
\end{tabular}

a Order of magnitude only. More exact values depend on instrumental details.

- May be modified chemically, by Langmuir-Blodgett film deposition, etc.

The lower limit of reported uncertainties $\Delta \Gamma$ appears to be $\Delta \Gamma \approx \mathrm{I} \mathrm{ng} / \mathrm{cm}^{2}$ (ellipsometry, IO). Techniques with $\Delta \Gamma$ a few fold greater fall into a second group (QCM, SAW devices, SAR, SPR). These figures compare with $\Gamma$ for a packed monolayer of a protein with a molecular weight $=100 \mathrm{kDa}$ of $\sim 500 \mathrm{ng} / \mathrm{cm}^{2}$. The most accurate techniques are therefore capable of resolving $0.2 \%$ of a monolayer, which is adequate for quantitative kinetic studies.

As mentioned in the introduction, very few truly comparative studies of the different techniques have been undertaken, i.e. those in which protein, substrate and solvent conditions were all identical. Some comparisons were made in order to calibrate the system (de Feijter et al. (1978) compared radiolabelling and ellipsometry; Stenberg et al. (1979) ellipsometry and impedance; Norde \& Rouwendal (1990) solution depletion and streaming potential; and Kulik et al. (I99I) TIRFM and radiolabelling) whereas other authors used the complementary aspects of the different techniques in order to elucidate the adsorption mechanism (Fair \& Jamieson ( 1980 ) compared solution depletion with QELS; Elgersma et al. (1992) compared streaming potential and SAR).

Speed of measurement. The techniques fall into three categories: off-line determination of $\Gamma$ (radiolabelling, BSD); those in which several measurements are needed to establish a dispersion curve which can then be fitted to a theoretical 
Table 2. Summary of working equations and assumed parameters

\begin{tabular}{|c|c|c|c|c|}
\hline Technique $^{a}$ & $\begin{array}{l}\text { Quantity } \\
\text { measured }\end{array}$ & Working equation ${ }^{b}$ & $\begin{array}{l}\text { Equation } \\
\text { numbers }^{c}\end{array}$ & $\begin{array}{l}\text { Assumed } \\
\text { parameters }\end{array}$ \\
\hline BSD & $c_{b}$ & $\frac{\left(c_{0}-c_{b}\right) V}{A}$ & 5 & None \\
\hline FD & $c_{2}$ & $\frac{\left(c_{0}-c_{b}\right) V}{A}$ & 6,5 & None \\
\hline CSD & $c_{b}$ & Transcendental & $7^{e}$ & $\begin{array}{l}\text { Form of } \\
\text { adsorption } \\
\text { isotherm }\end{array}$ \\
\hline $\left.\begin{array}{l}\text { QCM } \\
\text { SAW }\end{array}\right\}$ & $\Delta f / f$ & $-\frac{\Delta f}{f} \rho_{\mathrm{F}} d_{\mathrm{F}}$ & 9 & None \\
\hline $\begin{array}{l}\text { Colloid } \\
\text { diffusitivity }\end{array}$ & $\mathscr{C}(\tau)$ & Transcendental ${ }^{f}$ & $18,11,13,14,1$ & $d_{\lambda}^{g}$ \\
\hline $\begin{array}{l}\text { Capillary } \\
\text { flow }\end{array}$ & $\frac{\mathscr{F}_{C}^{\prime}}{\mathscr{F}_{\mathrm{c}}}$ & $R-\left(\frac{R^{4} \eta_{\mathrm{C}}^{\prime} \mathscr{F}_{\mathrm{C}}^{\prime}}{\eta_{\mathrm{c}} \mathscr{F}_{\mathrm{C}}}\right)^{\frac{1}{4}}$ & $22,23^{h}$ & ${ }^{h}$ \\
\hline MIC & $C$ & $\frac{\epsilon_{A}\left(C_{0 x}-C\right)}{C_{o x} C}$ & $33,34^{h}$ & $\epsilon_{A}{ }^{h}$ \\
\hline $\mathrm{SCI}$ & $C_{s c}$ & $\left(\frac{\epsilon_{s c} N_{D} e^{2}}{2 C_{s c}^{2}}-V_{d}\right) \frac{C_{\mathrm{A}}}{q_{p}}$ & $37,36,38,24$ & $C_{\mathrm{A}}, q_{p}$ \\
\hline FET & $I_{D}$ & $\left(\frac{I_{D} L}{W \mu C_{o x} V_{D}}+V_{T}-V_{G}\right) \frac{C_{\mathrm{A}}}{q_{p}}$ & $42,43,3^{8}, 24$ & $C_{A}, q_{p}$ \\
\hline IFCM & $V_{p}$ & $\frac{C_{\mathrm{A}} V_{p}}{q_{p}}$ & 38,24 & $C_{\mathrm{A}}, q_{p}$ \\
\hline $\begin{array}{l}\text { Ellipsometry } \\
\text { SAR } \\
\text { IO }\end{array}$ & $\begin{array}{l}\Delta, \psi \\
I_{p, r} / I_{p . i} \\
\alpha\end{array}$ & $\begin{array}{l}\text { Transcendental } \\
\text { Transcendental } \\
\text { See text }\end{array}$ & $\begin{array}{l}69,60,53,54,57 \\
60,54,57 \\
74,73,57\end{array}$ & $\begin{array}{l}\text { None } \\
\text { None } \\
\text { None }\end{array}$ \\
\hline $\begin{array}{l}\text { SPR } \\
\text { TIRFM }\end{array}$ & 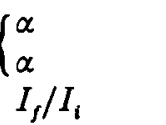 & $\begin{array}{l}\text { See text }{ }^{h} \\
\text { Transcendental } \\
K_{f}^{\prime \prime} I_{f} / I_{l}\end{array}$ & $\begin{array}{l}83,8 \mathrm{I}, 78 \\
83,8 \mathrm{I}, 78,57 \\
84\end{array}$ & $\begin{array}{l}n_{A} \\
\text { None } \\
K_{f}^{\prime \prime}\end{array}$ \\
\hline
\end{tabular}

a See appendix A for key to acronyms.

- To obtain $\Gamma$ (where $\theta$ is the primary quantity determined) the use of equation I is indicated in column 4 .

' In text.

d That is, all the quantities used in the working equation which cannot be measured independently, or from measurement prior to adsorption.

- Another equation linking $\Gamma$ with $c_{b}$ is required.

$\checkmark$ Method only useful for $\theta<0.5$.

- May be obtainable from the intensity of scattered light (equation 20).

${ }^{h}$ Method gives $d_{\mathrm{A}}$ only

' $K_{f}^{\prime}=K_{f} \int_{0}^{\infty} c(z) \phi(c) \exp \left(-z / \Delta z_{\mathrm{F}, \mathrm{C}}^{\prime}\right) d z$. 


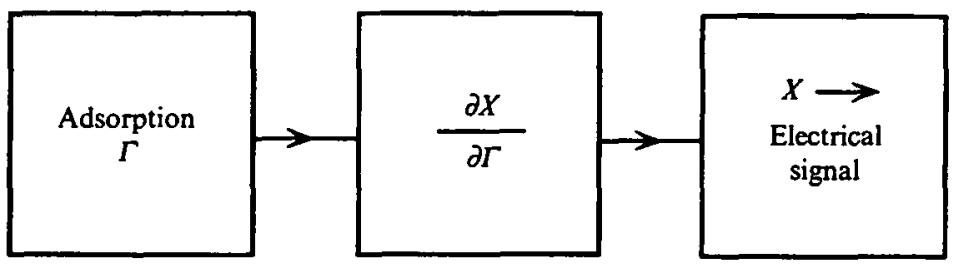

Fig. 24. Idealized measurement procedure.

curve to calculate $\Gamma$ (QELS, SAR, SPR, TIRS) ${ }^{25}$ and those in which a single simultaneous measurement suffices to determine $\Gamma$ (CSD, FD, electrical methods, QCM, SAW devices, ellipsometry, IO, TIRFM). The rapidity with which successive values of $\Gamma$ can be measured not only affects the kinetic range of processes which can be studied, but also determines the number of points available for fitting to kinetic models.

Hydrodynamic control. A detailed analysis of protein adsorption kinetics can only be carried out if the hydrodynamic conditions at and near the interface are known. Exact formulae are available for laminar flow across a planar surface and the rotating disk (Levich, 1962). Table I gives details of the morphology of substrates used with the different techniques. Since the conformation, orientation and arrangement of proteins at the surface has been found to depend on the flux of molecules to the surface ( $\left.\xi_{1} 0\right)$, the conditions most amenable to kinetic analysis are those in which the flux is constant, i.e. constant flow or rotation rate. This condition is also generally applied in numerical simulations of adsorption. Illdefined hydrodynamics are a major disadvantage of methods requiring comminuted material as substrate.

Calculation procedure. The techniques fall into the two categories of those in which $\Gamma$ can be calculated directly from the measured quantities (possibly making use of a null measurement prior to adsorption to establish the values of certain parameters), and those in which the values of a number of parameters must be assumed (for details see Table 2). Other things being equal, methods requiring the fewest assumptions are obviously preferable. A difficulty with calibrating the relative response from methods such as TIRFM and RET, for example by using radiotracers, is that the correspondence between absolute $\Gamma$ and the recorded signal (fluorescence intensity) may be nonlinear, and calibration over the entire range of expected conditions is needed if the results are to be considered reliable.

Versatility of substrate choice. The materials requirements of the different techniques are summarized in Table 1 . Methods are available for preparing small, uniform, monodisperse spheres from a wide variety of materials for use with the solution depletion and hydrodynamic methods. The electronics and optoelectronics industries have raised the technology of preparation of planar

${ }^{25}$ Provided certain assumptions are made (see the detailed discussion on each technique) a single measurement can give an approximate value of $\Gamma$. In principle, points acquired by scanning could be simultaneously collected using an optical multichannel analyzer. 
substrates usable with most of the other methods to a very high level of perfection. An advantage is enjoyed by techniques in which the adsorbing element is detachable and can readily be replaced or subjected to chemical modification (including cleaning) off-line, and which are suitable substrates for the deposition of Langmuir films, which is especially useful when investigating bilayer lipid membranes as the adsorbing surface (Cuypers et al. I 983; Ramsden \& Schneider, 1993). Planarity is desirable for ease of hydrodynamic analysis (see above); the possible chemical heterogeneity of typical surfaces has hitherto scarcely been considered; Kulik et al. (I99I) explicitly took surface heterogeneity into account in interpreting their results and found that the adsorption of serum albumin onto amorphous quartz was best described using a continuum of adsorption energies.

During the past few decades over which protein adsorption has been intensively studied, there has been a clear evolution from the early methods based on particles and capillaries to direct techniques using very well defined hydrodynamic conditions and planar substrates. I envisage this trend to continue. Optical methods (especially SAR, IO) should become the methods of choice for determining not only $\Gamma$ but also mapping out density profiles perpendicular to the surface; we can therefore expect to see an extension of these techniques and their associated calculation methods (e.g. simultaneous measurement of more than two guided modes, use of several wavelengths ${ }^{26}$ ). The capability of investigating a density profile perpendicular to the surface is especially important for the investigation of protein multilayer formation; techniques such as radiolabelling measure the total amount adsorbed and cannot distinguish between a dense monolayer and sparse multilayers. If $\Gamma, d_{\mathrm{A}}$ and $c_{\mathrm{A}}$ are available, other techniques will be valuable for yielding complementary information such as the charge distribution at the interface (electrical methods) protein-substrate bonding patterns (TIRS), lateral mobility of adsorbed proteins (TIRFM), and competitive adsorption and desorption (radiolabelling). One may also expect the growing application of scanning force microscopies to study protein adsorption problems.

Since reproducibility of the state of the surface is one of the major difficulties in investigating adsorption, I expect that the development of devices capable of measurements on the same substrate using several techniques simultaneously to become a priority. This should enable significant new insights into the conformation and activity of adsorbed layers.

\section{IO. THE NATURE OF THE ADSORBED PROTEIN LAYER}

There are a number of important basic questions in connexion with protein adsorption, the answers to which are still contentious. The first is how much area does an adsorbed protein occupy? Phenomenonologically it is fairly well established that, depending on the protein, and surface and solution conditions, the area per molecule is inversely proportional to the rate of arrival of proteins at the surface. In early work, this was manifested by the amount irreversibly adsorbed

26 Aspnes (1985) describes variable wavelength ellipsometry. 
being proportional to the starting solution concentration. More recently the phenomenon has been precisely demonstrated kinetically (Ramsden, I $993 b$ ).

The increased area adsorbed from a low solution concentration was often interpreted as a conformational change-denaturation. Circular dichroism spectroscopy showed that fibrinogen desorbed from glass had lost about half its $\alpha$ helical content (Chan \& Brash, 198r). Langmuir \& Schaefer (1938) suggested, however, that the loss of activity of proteins redispersed from an adsorbed monolayer was due to the destruction rather than the formation of the adsorbed film. Indeed the widespread use of solid phase immunoassays, which require that an adsorbed monolayer retains antigenic activity, makes it improbable that denaturation upon adsorption is a general phenomenon. Langmuir \& Schaefer also found that urease deposited on a hydrophobic surface lost its activity, but not when deposited on a hydrophilic one, but it is not necessary to invoke denaturation to explain the loss of activity, which could merely be due to the active site being oriented next to the surface and hence inaccessible from solution. Adsorbed proteins may be subject to strong forces, to which dynamic properties will be especially susceptible. For example, the photocycle of bacteriorhodopsin adsorbed on glass runs about 3 orders of magnitude more slowly than in suspended purple membrane fragments (I. V. Chizhov \& B.V. Zubov, unpublished observations).

The variation in occupied area with adsorption rate could have a simple geometric interpretation. Most globular proteins are spheroidal rather than spherical. Clearly a prolate spheroid ${ }^{27}$ may initially land with either its major or minor axes perpendicular to the surface (orientations $a_{\perp}$ and $b_{\perp}$ respectively). In the former case the occupied area will be $b^{2}$, in the latter case $a b$. The larger area will, as a rule, be more stable, and the transition $a_{\perp} \rightarrow b_{\perp}$, but not its converse, will be possible. The crowding of molecules at the surface, such as occurs during adsorption from a concentrated solution, will hinder the process $a_{\perp} \rightarrow b_{\perp}$ (which of course takes a finite time), and hence favour $a_{\perp}$.

Another question is whether proteins are irreversibly adsorbed. Dilution of the protein solution in contact with the surface generally results in the partial removal of material (e.g. Fig. 23), and the removable fraction dwindles as adsorption proceeds. Adsorption to many kinds of surface takes place via multiple weak bonds, whose number will be roughly proportional to the contact areas. Suppose that the $a_{\perp}$ orientation allows 5 bonds of energy $\sim k T$ each to form. ${ }^{28}$ Desorption requires all the bonds to be broken simultaneously, which has a probability $\sim e^{-5} \approx 7 \times 10^{-3}$. If $a=2 b$, the $b_{\perp}$ orientation will have about 10 weak bonds to the surface, with a corresponding probability of desorption of $5 \times 10^{-5} .^{29}$ Typically, the reversibly bound fraction $\left(a_{1}\right)$ desorbs over a time scale of $10 \mathrm{~min}$ (see Fig. 23); this means that the 'irreversible' fraction $\left(b_{\perp}\right)$ would desorb over $24 \mathrm{~h}$, and hence be unobservable during the $\mathrm{I}-2 \mathrm{~h}$ duration of typical experiments.

27 The same arguments apply, mutatis mutandis, to oblate spheroids.

28 At present, we do not really know if this is a reasonable number. Morrissey \& Stomberg (1974) found about 70 carbonyl surface contacts per adsorbed albumin molecule.

29 Since protein surfaces are heterogeneous, the number of bonds will depend on the orientation at the surface as much as on the contact area. 
The multiple weak bond model is also consistent with the observed displacement of adsorbed proteins by proteins in solution (Lutanie et al. 1992), ${ }^{30}$ and by high concentrations of small ions. The elementary treatment of the desorption probability adumbrated above is scarcely adequate, however, and more careful analysis is required. Inter alia, correlations between the breaking and reformation of bonds must be taken into account; adjacent bonds do not act independently. Whether transitions such as $a_{\perp} \rightarrow b_{\perp}$ indeed take place at the surface is contentious. In the work illustrated in Fig. 23 (Kurrat et al. 1993) the kinetics were not consistent with the conversion of the 'reversible' to the 'irreversible' adsorbed form at the surface; instead, separate adsorption pathways for the two forms had to be adopted as illustrated in Fig. 21. Since a molecule which is released from the surface will attempt to readsorb several times before diffusing away, due to confinement by the solvent molecules (in analogy to the cage effect (Rabinowitch, 1937) in homogeneous solution) the most efficient path from $a_{1}$ to $b_{\perp}$ may be via desorption, re-orientation in solution, and re-adsorption.

Non-wettable (hydrophobic) surfaces present a different problem. Soluble globular proteins have a low affinity for such surfaces relative to hydrophilic ones, but even brief residence on the surface exposes them to the possibility of unfolding to bring their hydrophobic interior in contact with surface. Once this transition has taken place the protein is effectively irreversibly adsorbed and denatured. Probably, the most benign substrate for adsorbing proteins is a lipid or glycolipid bilayer. Proteins anchored to such a surface via a hydrophobic moiety such as a short sequence of hydrophobic amino acids or a lipid tail which can penetrate the bilayer are likely to suffer the least structural change and fully retain their static structure and dynamic activity.

Ordering of proteins at the surface. The Schaaf-Talbot expression (92) assumes that proteins at the surface are maximally disordered - the random sequential adsorption (RSA) model, which has been shown to accurately describe the kinetics. The model is now being extended to more elongated shapes (Viot $e t$ al. 1992; Ricci et al. 1992). The influence of specific lateral interactions has not yet been investigated theoretically in dimensions higher than $I$. Whereas repulsive interactions may be approximated by simply taking an area per adsorbed molecule appropriately greater than the projected geometric area, attractive interactions may lead to condensation and two-dimensional crystallization, which may be revealed in the adsorption kinetics by a breakdown of the RSA model, although rules for interpretating such breakdowns are not yet established. By analyzing the hydrodynamic thicknesses of adsorbed layers at different $\Gamma$, Fair \& Jamieson (1980) provided convincing evidence for the two dimensional crystallization of bovine serum albumin at polystyrene surfaces. The behaviour of this protein is a typical example of low amounts adsorbed from dilute solutions, and higher amounts from concentrated ones. Morrissey \& Han (1978) had previously: interpreted this well-established result as indicating two conformations, the flatter one occupying a greater area being favoured at low bulk protein concentration.

\footnotetext{
30 These workers found that immunoglobulin adsorbed on a silica surface by displacing previously adsorbed albumin lost its antigenic activity, but not when it was adsorbed directly.
} 
It has been aptly pointed out that 'the literature pertaining to the study of protein adsorption at solid-liquid interfaces has a long and confusing history' (Fair \& Jamieson, I 980). Despite the great volume of work carried out over the past decades, general rules scarcely exist and, given the astonishing variety of known proteins, one should beware of applying results derived from observations made with a few different proteins to proteins in general.

Reliable and convenient experimental techniques are an essential prerequisite for progress to be made in unravelling the mysteries of protein adsorption. With a number of such techniques now available, prospects for elucidating laws predicting adsorption kinetics and equilibrium behaviour, knowing only the protein structure, the chemistry and morphology of the substrate, and the solution conditions, are excellent.

\section{I. ACKNOWLEDGMENT}

It is a pleasure to record my thanks to Mrs M. Jäggi for having drawn the figures.

\section{REFERENCES}

Agranovich, V. M. \& Mills, D. L. (1982). Surface Polaritons. Amsterdam: NorthHolland.

Alexandrowicz, Z. \& Daniel, E. (1963). Sedimentation and diffusion of polyelectrolytes. Biopolymers $\mathrm{x}, 447-47 \mathrm{I}$.

Alvarez, O. \& LATORRE, R. (1978). Voltage-dependent capacitance in lipid bilayers. Biophys. F. 21, 1-17.

ANdrade, J. D. \& Hlady, V. L. (1986). Protein adsorption and materials biocompatibility. Adv. Polymer Sci. 79, I-63.

Andrade, J. D., Hlady, V. L. \& van Wagenen, R. A. (1984). Effects of plasma protein adsorption on protein conformation and activity. Pure appl. Chem. 56, I345-1 $35^{\circ}$.

Aptel, J. D., Voegel, J. C. \& Schmitt, A. (1988). Adsorption kinetics of proteins onto solid surfaces in the limit of interfacial interaction control. Colloids Surf. 29, 359-371.

Artmann, K. (1948). Berechnung der Seitenversetzung des totalrefelktierten Strahles. Ann. Phys. 2, 87-102.

Aspnes, D. E. (1982). Optical properties of thin films. Thin Solid Films 89, 249-262.

ASPNES, D. E. (1985). Recent progress in the nondestructive analysis of surfaces, thin films and interfaces by spectroellipsometry. Appl. Surf. Sci. 22/23, 792-803.

AXelrod, D., BurghardT, T. P. \& Thompson, N. L. (1984). total internal reflexion fluorescence. A. Rev. Biophys. Bioengng 13, 247-268.

Azzam, R. M. A. \& Bashara, N. M. (1977). Ellipsometry and Polarized Light. Amsterdam: North Holland.

de Baillou, N., Dejardin, P., Schmitt, A. \& Brash, J. L. (1984). Fibrinogen dimensions at an interface. $\mathcal{F}$. Colloid Interface Sci. 100, I67-174.

BARD, A. J. \& FAULKNER, L. R. (1980). Electrochemical Methods. New York: Wiley.

Bartelt, M. C. \& Privman, V. (1991). Kinetics of irreversible monolayer and multilayer adsorption. Int. F. mod. Phys. B 5, 2883-2907.

Bernabeu, P., de Cesare, A. \& CAPrani, A. (1989). Kinetics of albumin and fibrinogen adsorption onto a rotating disk electrode. $\mathcal{F}$. electroanal. Chem. 265, 261-275. 
Berne, B. J. \& Pecora, R. (1975). Dynamic Light Scattering. New York: Wiley.

Bloomfield, V., Dalton, W. O. \& van Holde, K. E. (1967). Frictional coefficients of multisubunit structures. Biopolymers 5, $135-148$.

Bockris, J. O'M., Devanathan, M. A. V. \& Müller, K. ( 1963$)$. Proc. R. Soc. Lond. A 274, 55-79.

BoDdy, P. J. (1965). The structure of the semiconductor-electrolyte interface. $\mathcal{F}$. electroanal. Chem. 10, 199-244.

Brown, R. (1828). A brief account of microscopical observations on the particles contained in the pollen of plants. Phil. Mag. (New Ser.) 4, 161-173.

CADY, W. G. (1946). Piezoelectricity. New York: McGraw-Hill.

Calabrese, G. S., Wohltjen, H. \& Roy, M. K. (1987). Surface acoustic wave devices as chemical sensors in liquids. Evidence disputing the importance of Rayleigh wave propagation. Analyt. Chem. 59, 833-837.

Caras, S. \& Janata, J. (1988). Enzymatically Sensitive Field Effect Transistors. In Methods in Enzymology (ed. Mosbach, K.), vol. 137, pp. 247-255. San Diego: Academic.

Chan, B. M. C. \& Brash, J. L. (1981). Conformational change in fibrinogen desorbed from glass surface. F. Colloid Interface Sci. 84, 263-265.

Chapman, D. L. (1913). A contribution to the theory of electrocapillarity. Phil. Mag. (Ser. 6) 25, 475-48I.

Charmet, J. C. \& DE Gennes, P. G. (1983). Ellipsometric formulae for an inhomogeneous layer with arbitrary refractive index profile. $\mathcal{F}$. opt. Soc. Am. 73, 1777-1784.

Cherny, V. V., Sokolov, V.S. \& ABidor, I. G. (1980). Determination of the surface charge of bilayer lipid membranes. Bioelectrochem. Bioenergetics $7,4^{1} 3^{-420}$.

Chittur, K. K., Fink, D. J., Leininger, R. I. \& Hutson, T. B. (I986). Fourier transform infrared spectroscopy/attenuated total reflexion studies of protein adsorption in flowing systems: approaches for bulk correction and compositional analysis of adsorbed and bulk proteins in mixtures. F. Colloid Interface Sci. 111, 419-433.

Chu, B. (1974). Laser Light Scattering. New York: Academic Press.

Cohen, R. R. \& Radke, C. J. (I99I). Streaming potentials of nonuniformly charged surfaces. $\mathcal{F}$. Colloid Interface Sci. r4I, 338-347.

Colowick, S.P. \& Womack, F.C. (1969). Binding of diffusible molecules by macromolecules: rapid measurement by rate of dialysis. F. biol. Chem. 244, 774-777.

Cornelius, R. M., Wojciechowski, P. W. \& Brash, J. L. (I992). Measurement of protein adsorption kinetics by an in situ, real-time, solution depletion technique. $\mathcal{F}$. Colloid Interface Sci. 150, I 2 I-1 33 .

Crank, J. (1975). The Mathematics of Diffusion. 2nd Edn. Oxford: Clarendon Press.

Cullen, D. C., Brown, R. G. W. \& Lowe, C. R. (1987/88). Detection of immunocomplex formation via surface plasmon resonance on gold-coated diffraction gratings. Biosensors 3, 2 I I-225.

Cummins, H. Z. \& Swinney, H. L. (1970). Light beating spectroscopy. Prog. Optics 8, I33-200.

Cummins, H. Z. \& PIke, E. R. (eds) (1974). Photon Correlation and Light Beating Spectroscopy. New York: Plenum.

Curie, J. \& Curie, P. (1890). Développement, par pression, de l'électricité polaire dans les cristaux hémièdres à faces inclinées. C. r. hebd. Séanc. Acad. Sci., Paris 91, 294-295.

Cuypers, P. A., Corsel, J. W., Janssen, M. P., Kop, J. M. M., Hermens, W. Th. \& 
Hemker, H.C. (1983). The adsorption of prothrombin to phosphatidylserine multilayers quantitated by ellipsometry. $\mathcal{F}$. biol. Chem. 258, 2426-2431.

DickMan, R., WANG, J.-S. \& JENSEN, I. (I991). Random sequential adsorption: series and viral expansions. F. chem. Phys. 94, 8252-8257.

Duckworth, D. S., LiPS, A. \& Staples, E. J. (1978). Concentration effects in polymer flocculation and stabilization. Faraday Discuss. chem. Soc. 65, 288-295.

van Dulm, P. \& NoRDE, W. (1983). The adsorption of human plasma albumin on solid surfaces, with special attention to the kinetic aspects. F. Colloid Interface Sci. 9r, 248-255.

VAN DUlm, P., NORDE, W. \& LYKLEMA, J. (1981). Ion participation in protein adsorption at solid surfaces. $\mathcal{F}$. Colloid Interface Sci. 82, 77-82.

Efimov, E. A. Erusalemchik, I. G. (1963). Electrochemistry of germanium and silicon. Washington, D.C.: Sigma Press.

Elgersma, A. V., Zsom, R. L. J., LyKlema, J. \& Norde, W. (1992). Kinetics of single and competitive protein adsorption studied by reflectometry and streaming potential measurements. Colloids Surf. 65, 17-28.

Engvall, E. \& Perlmann, P. (1971). Enzyme-linked immunosorbent assay (ELISA). Immunochemistry 8, 87I-874.

Ermakov, Yu. A., Fevraleva, I.S. \& Attaullakhanov R. I. (1985). Влияниe поликатионов на граничные потенциалы бислойных липидных мембран. Биологические Мембраны 2, 1094-1 100.

Ermakov, Yu. A. (1990). The determination of binding site density and association constants for monovalent cation adsorption onto liposomes made from mixtures of zwitterionic and charged lipids. Biochim. biophys. Acta 1023, 9 I-97.

Fahrenfort, J. (1961). Attenuated total reflexion. Spectrochim. Acta 17, 698-709.

FAIR, B. D. \& JAMIESON, A. M. (I980). Studies of protein adsorption on polystyrene latex surfaces. F. Colloid Interface Sci. 77, 525-534.

de Feijter, J. A., Benjamins, J. \& Veer, F. A. (1978). Ellipsometry as a tool to study the adsorption behaviour of polymers at the air-water interface. Biopolymers 17, 1759-1772.

Feldman, .K. (1978). New devices for flow dialysis and ultrafiltration for the study of protein-ligand interactions. Analyt. Biochem. 88, 225-235.

FörSTER, TH. (1948). Zwischenmolekulare Energiewanderung und Fluoreszenz. Ann. Physik (6. Folge) 2, 55-75.

Gebbert, A., Alvarez-Icaza, M., Stöcklein, W. \& Schmid, R. D. (1992). Real-time monitoring of immunochemical interactions with a tantalum capacitance flowthrough cell. Analyt. Chem. 64, 997-1003.

Ghatak, A. K. \& Thyagarajan, K. (1989). Optical Electronics. Cambridge: Cambridge University Press.

Gobrecht, H. \& MeInhardt, O. (1963). Über die Impedanz von HalbleiterElektrolyt-Grenzflächen. Ber. BunsenGes. phys. Chem. 67, 142-15I.

Goos, F. \& HäncheN, H. (1947). Ein neuer und fundamentaler Versuch zur Totalreflexion. Ann. Physik (6. Folge) x, 333-346.

Goos, F. \& LiNDBERG-HäNCHEN, H. (1 950). Neumessung des Strahlversetzungseffektes bei Totalreflexion. Ann. Physik (6. Folge) 5, 25 I-252.

Gouy, M. ( 1910 ). Sur la constitution de la charge électrique à la surface d'un électrolyte. 7. Phys. Radium 9, 457-468.

GraY, R. W. \& RAMSAY, W. (1909). Some physical properties of radium emanation. $\mathcal{F}$. chem. Soc. 95, 1073-1085. 
HARRICK, N. J. (1960). Surface chemistry from spectral analysis of totally internally reflected radiation. Phys. Rev. Lett. 4, 224-226; Y. phys. Chem. 64, I I I0-III4.

HaRRICK, N. J. (1967). Internal Reflexion Spectroscopy. New York: Wiley.

Harten, H. U. (1964). Die Grenzfläche Halbleiter-Elektrolyt. In Festkörperprobleme, Band III (ed. Sauter, F.), pp. 81-123. Brunswick: Vieweg.

Healy, T. W. \& White, L. R. (1978). Ionizable surface group models of aqueous interfaces. Adv. Colloid Interface Sci. 9, 303-345.

HerRmanN, P. P. \& Wildmann, D. (1983). Fabrication of planar dielectric wave-guides with high optical damage threshold. IEEE $\mathcal{~ Y . ~ Q u a n t u m ~ E l e c t r o n . ~ Q E - 1 9 , ~ 1 7 3 5 - 1 7 3 8 . ~}$

HeUberger, K. \& LUKosz, W. (1986). Embossing technique for fabricating surface relief gratings on hard oxide waveguides. Appl. Opt. 25, 1499-1 504.

HLADY, V. (1991). Spatially resolved adsorption kinetics of immunoglobulin $\mathrm{G}$ onto the wettability gradient surface. Appl. Spectrosc. 45, 246-252.

Hunter, R. J. (1981). Zeta Potential in Colloid Science. London: Academic Press.

Iwamoto, G. K., Winterton, L. C., Stoker, R. S., van Wagenen, R. A., Andrade, J. D. \& Mosher, D. F. (1985). Fibronectin adsorption detected by interfacial fluorescence. F. Colloid Interface Sci. 106, 459-464.

Jenkins, F. A. \& White, H. E. (r950). Fundamentals of Optics, and ed., New York: McGraw-Hill.

Johnson, J. E. \& Matijević, E. (1992). Interactions of proteins with uniform colloidal hematite and chromium hydroxide particles. I. Adsorption. Colloid Polym. Sci. 270, $353-363$.

JONES, R. C. (1952). 'Detectivity': the reciprocal of noise equivalent input of radiation. Nature, Lond. 170, 937-938.

KALB, E. \& ENGEL, J. (I 991 ). Binding and calcium-induced aggregation of laminin onto lipid membranes. F. biol. Chem. 266, 19047-19052.

Kanazawa, K. K. \& GoRdon, J. G. (1985). The oscillation frequency of a quartz resonator in contact with a liquid. Analytica chim. Acta 175, 99-105.

KolB, D. M. (1982). The study of solid-liquid interfaces by surface plasmon polariton excitation. In Agranovich \& Mills, pp. 299-329.

Kopaciewicz, W., Rounds, M. A., Fausnaugh, J. \& Regnier, F. E. (1983). Retention model for high-performance ion-exchange chromatography. I. Chromatogr. 266, 3-2I.

Kretschmann, E. (1971). Die Bestimmung optischer Konstanten von Metallen durch Anregung von Oberfiächenplasmaschwingungen. Z. Phys. 24I, 31 3-324.

Kulik, E. A., Kalinin, I. D. \& Sevastyanov, B. I. (1991). Кинетика адсорбции сывороточного альбумина на поверхности аморфного кварца. Журнал физической Химии 65, 2230-2234.

Kulik, E. A. \& Sevastyanov, B. I (г991). Различия в энергиях взаимодействия белок/поверхность и структурные изменения адсорбированных белков. Журнал бизической Химии 65, 2234-2239.

Kuramitsu, S. \& Hamaguchi, K. (1980). Analysis of the acid-base titration curve of hen lysozyme. F. Biochem. 87, 1215-1219.

Kurrat, R., Ramsden, J. J. \& Prenosil, J. E. (i 993 ). Kinetic model for serum albumin adsorption: experimental verification. F. chem Soc. Faraday Trans. (in press).

Laane, C., Willner, I., Otvos, J. W. \& Calvin, M. (198I). Photosensitized electron transfer processes in $\mathrm{SiO}_{2}$ colloids and sodium lauryl sulfate micelles. Proc. natn. Acad. Sci. U.S.A. 78, 5928-5932. 
LANGmuir, I. \& Schaefer, V. J. (1938). Activities of urease and pepsin monolayers. $\mathcal{F}$. Am. chem. Soc. 60, $1351-1360$.

LAW, B. M. \& BeAGLeHOLE, D. (198I). Model calculations of the ellipsometric properties of inhomogeneous dielectric surfaces. F. Phys. D 14, 11 5-126.

LEAver, G., Howell, J. A. \& CONDER, J. R. (1992). Adsorption kinetics of albumin on a cross-linked chromatographic ion exchanger. F. Chromatogr. 590, 101-I I 2.

Lecompte, M. F., Clavilier, J., Dode, C., Elion, J. \& Miller, I. R. (I984). Electroactivity of adsorbed prothrombin at the $\mathrm{Hg}$-solution interface. $\mathcal{F}$. electroanal. Chem. 163, 345-362.

LeE, R. G. \& WeBer, T. W. (1969). Isothermal adsorption in fixed beds. Can. F. Chem. Engng 47, 54-59.

Levich, V. G. (1962). Physicochemical Hydrodynamics. Englewood Cliffs, NJ : Prentice Hall.

LIN, B. \& Rice, S. A. (1993). Evanescent wave light scattering study of a diblock copolymer adsorbed at the air/water interface. F. chem. Phys. 98, 6561-6562.

LuKosz, W. (1991). Principles and sensitivities of integrated optical and surface plasmon sensors for direct affinity sensing and immunosensing. Biosensors Bioelectronics 6 , $215-225$.

Lukosz, W., Nellen, Ph. M., Stamm, Ch. \& Weiss, P. (1990). Output grating couplers on planar waveguides as integrated optical chemical sensors. Sensors Actuators Br, 585-588.

Lukosz, W. \& Stamm, Сн. (1991). Integrated optical interferometer as relative humidity sensor and differential refractometer. Sensors Actuators A 25-27, 185-188.

Lukosz, W. \& TiEfenthaler, K. (1983). Embossing technique for fabricating integrated optical components in hard inorganic waveguiding materials. Optics Lett. 8, 537-539.

Lundström, I. \& Elwing, H. (1990). Simple kinetic models for protein exchange reactions on solid surfaces. $\mathcal{~}$. Colloid Interface Sci. 136, 68-84.

Lutanie, E., Voegel, J. C., Schaaf, P., Freund, M., Cazenave, J. P. \& Schmitt, A. (1992). Competitive adsorption of human immunoglobulin $\mathrm{G}$ and albumin. Proc. natn. Acad. Sci. U.S.A. 89, 9890-9894.

MacRitiche, F. (1978). Proteins at interfaces. Adv. Protein Chem. 32, 283-326.

MANGelsDorf, C. S. \& White, L. R. (1990). Effects of stem-layer conductance on electrokinetic transport properties of colloidal particles. F. chem. Soc. Faraday Trans. 86, 2859-2870.

Mason, W. P., Baker, W. O., McSkimin, H. J. \& Heiss, J. H. (1949). Measurement of shear elasticity and viscosity of liquids at ultrasonic frequencies. Phys. Rev. 75 , 936-946.

Masuda-Nakagawa, L. M. \& WiedemanN, C. (I992). The rôle of matrix molecules in regeneration of leech central nervous system. $\mathcal{F}$. Neurobiol. 23, 551-567.

Maternaghan, T.J. \& Ottewill, R. H. (1974). An ellipsometric study of the adsorption of gelatin on silver bromide. $\mathcal{F}$. photogr. Sci. 22, 279-285.

McCammon, J. A., Deutch, J. M. \& Felderhof, B. U. (1975). Frictional properties of multisubunit structures. Biopolymers 14, 2613-2623.

McCrackin, F. L., Passaglia, E., Stromberg, R. R. \& Steinberg, H. L. (1963). F. Res. natn. Bur. Standards A 67, 363-377.

Morrissey, B. W. \& Stromberg, R. R. (1974). The conformation of adsorbed blood proteins by infrared bound fraction measurements. $\mathcal{F}$. Colloid Interface Sci. 46, $152-164$.

Morrissey, B. W., Smith, L. E., Stromberg, R. R. \& Fenstermaker, C. A. (1976). 
Ellipsometric investigation of the effect of potential on blood protein conformation and absorbance. 7 . Colloid Interface Sci. 56, 557-563.

MoRRISSEY, B. W. \& HAN, C. C. (1978). The conformation of $\gamma$-globulin adsorbed on polystyrene latices determined by quasielastic light scattering. $\mathcal{F}$. Colloid Interface Sci. 65, 423-43I.

Mura-Galelli, M. J., Voegel, J. C., Behr, S., Bres, E. F. \& Schaaf, P. (ig9i). Adsorption/desorption of human serum albumin on hydroxyapatite : a critical analysis of the Langmuir model. Proc. natn. Acad. Sci. U.S.A. 88, 5557-5561.

Myamlin, V.A. \& Pleskov, Yu. V. (1967). Electrochemistry of Semiconductors. New York: Plenum Press.

Nakata, S., Yoshikawa, K. \& Matsuda, T. (1992). Voltage-dependent capacitance as a probe for albumin adsorption onto a solid surface. Biophys. Chem. 42, 21 3-220.

Newton, I. (1687). Philosophiae naturalis principia mathematica. Lib. I, Sect. XIV, Prop. XCVI, Theor. L. London: The Royal Society.

Nieto, A., GayÁ, A., Moreno, C., Jansá, M. \& Vives, J. (I 986). Adsorption-desorption of antigen to polystyrene plates used in Elisa. Ann. Inst. Pasteur/Immunol. $137 \mathrm{C}$, $161-172$.

NORDE, W. (1986). Adsorption of proteins from solution at the solid-liquid interface. Adv. Colloid Interface Sci. 25, 267-340.

Norde, W. \& LyKLEMA, J. (1978). The adsorption of human plasma albumin and bovine pancreas ribonuclease at negatively charged polystyrene surfaces. $\mathcal{F}$. Colloid Interface Sci. 66, 257-302.

Norde, W. \& LyKLema, J. (1979). Thermodynamics of protein adsorption. F. Colloid Interface Sci. 71, 350-366.

NoRdE, W. \& Rouwendal, E. (1990). Streaming potential measurements as a tool to study protein adsorption kinetics. F. Colloid Interface Sci. 139, 169-1 76.

NyGReN, H. (1992). Critical dissociation of ferritin during adsorption at a liquid-solid interface. Prog. Coll. Polymer. Sci. 88, 86-89.

Nygren, H. \& Karlsson, C. (1992). Intermolecular interaction and ordering of fibrinogen at a liquid-solid interface. Prog. Coll. Polymer. Sci. 88, 96-99.

Nygren, H. \& STENBERG, M. (1990a). Surface-induced aggregation of ferritin. Bioplyys. Chem. 38, 67-75.

Nygren, H. \& Stenberg, M. (1990b). Surface-induced aggregation of ferritin. Biophys. Chem. 38, 77-85.

O'Brien, R. W. \& White, L. R. (I 978). Electrophoretic mobility of a spherical colloidal particle. Y. chem. Soc. Faraday Trans. 2 77, 1607-1626.

van den Oetelatr, P. J. M., Mentink, I. M. \& Brinks, G. J. (1989). Loss of peptides and proteins upon sterile filtration due to adsorption to membrane filters. Drug Development ind. Pharmacy 15, 97-106.

Paik, W.-K., Genshaw, M. A. \& Bockris, J. O'M. (I970). The adsorption of anions at the solid-solution interface. F. phys. Chem. 74, 4266-4275.

Place, H., Sébille, B. \& Vidal-Madjar, C. (I991). Characterization of the adsorption kinetics of proteins on reversed-phase supports. Analyt. Chem. 63, 1 222-1 227.

Pleskov, Yu. V. \& Gurevich, YU. YA. (1986). Semiconductor Photoelectrochemistry. New York: Plenum Press.

Popkirov, G. \& TABOv, N. (1982). Method for measurement of capacitance, series and shunt resistances of semiconductor junctions. Rev. scient. Instrum. 53, 864-866.

Porstmañ , T. \& Kiessig, S. T. (1992). Enzyme immunoassay techniques. F. Immun. Meth. 150, 5-21. 
Rabinowitch, F. (1937). Collision, coördination, diffusion and reaction velocity in condensed systems. Trans. Faraday Soc. 33, 1225-1233.

RAmSDEN, J. J. (1985). Nucleation and growth of small CdS aggregates by chemical reaction. Surf. Sci. 156, 1027-1039.

RAMSDEN, J. J. (1987). Electron diffraction anomalies in small CdS clusters. F. Cryst. Growth 82, 569-572.

RAMSDEN, J.J. (1993a). Calcium-dependence of laminin binding to phospholipid monolayers. Biopolymers 33, 475-477.

Ramsden, J. J. (1993b). Concentration scaling of protein deposition kinetics. Phys. Rev. Lett. 7I, 295-298.

RAMSDEN, J. J. (1993c). Review of new experimental techniques for investigating random sequential adsorption. $\mathscr{F}$. statist. Phys. 73, 853-877.

Ramsden, J. J. \& Prenostl, J. E. (1993). Salt effects on protein adsotption kinetics. (Submitted).

RAMSDEN, J. J. \& SCHNEIDER, P. (I993). Membrane insertion and antibody recognition of a glycosylphosphatidylinositol-anchored protein. Biochemistry 32, 523-529.

ReInders, W. \& DE Minjer, C. H. (1938). The redox potentials of complex iron salts with the sodium salts of organic acids. Recl Trav. chim. Pays-Bas Belg. 57, 594-603.

Ricci, S. M., TAlbot, J., Tarjus, G. \& Viot, P. (1992). Random sequential adsorption of anisotropic particles. II. Low coverage kinetics. F. chem. Phys. 97, 5219-5228.

Righetri, P. G., Tudor, G. \& EK, K. (r98I). Isoelectric points and molecular weights of proteins. $\mathcal{F}$. Chromatogr. 220, I I 5-I 94.

Rocco, M., Infusini, E., Daga, M. G., Gogioso, L. \& Cuniberti, C. (1987). Models of fibronectin. EMBO F. 6, 2343-2349.

Roederer, J. E. \& BastiaAns, G. J. (I983). Microgravimetric immunoassay with piezoelectric crystals. Analyt. Chem. 55, 2333-2336.

Rondelez, F., AusSerrÉ, D. \& Hervet, H. (1987). Experimental studies of polymer concentration profiles at solid-liquid interfaces by optical and X-ray evanescent wave techniques. A. Rev. phys. Chem. 38, 317-347.

Rowland, F. W. \& Eirich, F. R. (1966a). Flow rates of polymer solutions through porous disks. 1. Method. F. Polymer Sci. A-1 4, 2033-2040.

Rowland, F. W. \& EIRICH, F. R. (1966b). Flow rates of polymer solutions through porous disks. 2. Thickness and structure of adsorbed polymer films. J. Polymer Sci. A-I 4, 240I-242I.

SARKar, D. \& Chattoraj, D. K. (1992). Absolute reaction rate and kinetics of protein adsorption at solid-liquid interfaces. Ind. F. Biochem. Biophys. 29, I 35-142.

SAUERBREY, G. ( 1959 ). Verwendung von Schwingquarzen zur Wägung dünner Schichten und zur Mikrowägung. Z. Phys. 155, 206-222.

Schaaf, P., Dejardin, Ph. \& Schmitt, A. (1985). Réflectométrie appliquée aux interfaces diffuses: possibilités et limites de la technique. Revue Phys. Appl. 20, $631-640$.

Schaaf, P. \& Dejardin, Ph. (1988). Structural changes within an adsorbed fibrinogen layer during the adsorption process. Colloids Surf. 31, 89-103.

Schaaf, P., Dejardin, Ph., Johner, A. \& Schmitt, A. (1987a). Thermal denaturation of an adsorbed fibrinogen layer studied by reflectometry. Langmuir 3, I I 28-I I 3 I.

Schaaf, P., Dejardin, Ph. \& Schmitt, A. (1987b). Reflectometry as a technique to study the adsorption of human fibrinogen at the silica-solution interface. Langmuir 3 , II $31-1135$. 
SchaAf, P. \& TAlbot, J. (1989). Surface exclusion effects in adsorption processes. $\mathcal{F}$. chem. Phys. 91, 4401-4409.

SchotTKY, W. (1942). Vereinfachte und erweiterte Theorie der Randschichtgleichrichter. $Z$. Phys. 118, 539-592.

Schumacher, R. (1990). The quartz microbalance. Angew. Chem. 102, 347-361; Angew. Chem. Int. Ed. 29, 329-3+3.

SinanoĞlu, O. (198I). What size cluster is like a surface? Chem. Phys. Lett. 81, I88-190.

Soderquist, M. E. \& WALton, A. G. ( 1980 ). Structural changes in proteins adsorbed on polymer surfaces. $\mathcal{F}$. Colloid Interface Sci. 75, 386-397.

SpIRo, T. (1985). Resonance Raman spectroscopy as a probe of heme protein structure and dynamics. Adv. Protein Chem. 37, I I I-1 59.

Stelzle, M. \& SACKmanN, E. (1989). Sensitive detection of protein adsorption to supported lipid bilayers by frequency-dependent capacitance measurements and microelectrophoresis. Biochim. biophys. Acta 981, 135-142.

Stenberg, M., Arwin, H. \& Nilsson, A. (1979). Silicon-silicon dioxide as an electrode for electrical and ellipsometric measurements of adsorbed organic molecules. $\mathcal{F}$. Colloid Interface Sci. 72, 255-264.

Stern-Hamburg, O. (1924). Zur Theorie der elektrolytischen Doppelschicht. $Z$. Elektrochem. 30, 508-516.

Stoner, G. \& SRINAvasan, S. (1970). Adsorption of blood proteins on metals using capacitance techniques. $\mathcal{F}$. phys. Chem. 74, I088-1094.

Strachan, C. (1933). The reflexion of light at a surface covered by a monomolecular film. Proc. Camb. phil. Soc. 29 (Part I), I I 6-1 30.

Swalen, J. D., Tacke, M., Santo, R., Rieckhoff, K. E. \& Fischer, J. (1 978). Spectra of organic molecules in thin films. Helv. chim. Acta 6r, 960-977.

SzE, S. M. (1981). Physics of Semiconductor Devices. 2nd Edition. New York: Wiley.

TAMm, L. \& Bartoldus, I. (1988). Antibody binding to lipid model membranes. Biochemistry 27, 7453-7458.

TiEfenthaler, K. (1992). Integrated optical couplers as chemical waveguide sensors. Adv. Biosensors 2, 261-289.

TiefenthaleR, K. \& Lukosz, W. (r 989 ). Sensitivity of grating couplers as integratedoptical chemical sensors. F. opt. Soc. Am. B 6, 209-220.

TIEN, P. K. ( 1977 ). Integrated optics and new wave phenomena in optical waveguides. Rev. mod. Phys. 49, 36I-420.

Tilton, R. D., Robertson, C. R. \& Gast, A. P. (1990). Lateral diffusion of bovine serum albumin adsorbed at the solid-liquid interface. F. Colloid Interface Sci. 137, I 92-203.

UzGIRIs, E. E. \& Fromageot, H. P. M. (1976). Thickness and density of protein films by optical mixing spectroscopy. Biopolymers $15,257^{-263}$.

VAšičEK, A. (1960). Optics of Thin Films. Amsterdam: North-Holland.

Verwey, E. J. W. \& Overbeek, J. Th. G. (1948). Theory of the Stability of Lyophobic Colloids. New York: Elsevier.

Veselova, M. N., Chukhrai, E. S. \& Poltorak, O. M. (1987). Кинетика адсорбции шелочной фосфатазы на модифицированном силикагеле. Журнал физической Химии 6r, 246I-2464.

Viot, P., Tarjus, G., Ricci, S. M. \& Talbor, J. (1992). Random sequential adsorption of anisotropic particles. I. Jamming limit and asymptotic behaviour. F. chem. Phys. 97 , $5212-5218$. 
VOGELSBERGER, W. \& MARX, G. (1976). Zur Krümmungsabhängigkeit der Oberflächenspannung kleiner Tröpchen. $Z$. phys. Chem. (Leipzig) 257, 580-586.

van Wagenen, R.A. \& ANDRADE, J. D. ( 1980 ). Flat plate streaming potential investigations: hydrodynamics and electrokinetic equivalency. $\mathcal{F}$. Colloid Interface Sci. 76, 305-314.

WARD, M. D. \& ButTRY, D. A. (I990). In situ interfacial mass detection with piezoelectric transducers. Science, N.Y. 249, 1000-1007.

White, R. M. (1970). Surface elastic waves. Proc. IEEE 58, 1238-1 276.

Wiersema, P. H., Loeb, A. L. \& Overbeek, J. Th. G. (1966). Calculation of electrophoretic mobility of a spherical colloid particle. F. Colloid Interface Sci. 22, 78-99.

Wreghitt, T. G. \& Morgan-CaPNer, P. (ed.) (1990). Elisa in the Clinical Microbiology Laboratory. London: Public Health Laboratory Service.

YEN, Y.-S. \& WONG, J. S. (1989). Infrared reflectance properties of surface thin films. J. phys. Chem. 93, 7208-7216.

ZweIG, H. J. (1964). Performance criteria for photodetectors. Photogr. Sci. Engng 8, 305-311.

\section{APPENDIX LIST OF PRINCIPAL SYMBOLS}

$a$

A

$\mathscr{A}$

$B$

$b$

$\mathscr{B}$

$c$

C

area per adsorbed protein molecule, Lambert absorption coefficient, length of major axis of prolate ellipsoid

amplitude, analyzer setting, area of adsorbing substrate

ratio of amplitudes

volume

propagation constant parallel to interface concentration

capacitance

autocorrelation function

thickness of a layer

Goos-Hänchen shift, translational diffusion coefficient

phase difference between $s$ - and $p$-polarizations

elementary (electron) charge

electric field strength

frequency

solution flow rate

acceleration due to gravity

activity of proton

height, magnetic field strength

current, light intensity

convective-diffusive flux of protein to the surface

Boltzmann's constant, modulus of wave vector, rate constant

miscellaneous constants, modulus of scattering vector

diffraction order

length

mass of a protein molecule, measuring interval, mode number

carrier concentration, refractive index 
doping density, effective refractive index ( $\equiv$ mode index) of waveguide, noise charge of an ion accumulated charge per unit area due to adsorption, figure of merit of resonator

distance measured perpendicular to the adsorbing plane 
u velocity

\section{Subscripts}

- initial, surface

I near surface

2 downstream

a adsorption

A adlayer (adsorbed proteins)

b bulk

B Brewster

C. cell, coherence

C cover medium (buffer solution)

$d$ depletion, desorption, diffusion boundary layer

$d d l \quad$ diffuse double layer

$D$ donor, doping, drain

$e \quad$ enrichment

$f \quad$ fluorescence

F solid film (substrate for adsorption)

$F \quad$ Förster

$G \quad$ gate

$i \quad$ incident beam, inversion

$j \quad$ particle or ion index

$L \quad$ load

M membrane, metal

ox oxide

$p \quad$ parallel to plane of reflexion, protein

$r \quad$ reflected beam

$s \quad$ perpendicular to plane of reflexion, scattered light, sticking (irreversibly to surface)

sc semiconductor

S support

$S \quad$ source

$T \quad$ threshold

TE transverse electric

TM transverse magnetic

\section{Acronyms}

BSD batch solution depletion

CSD continuous solution depletion

FD flow dialysis

FET field effect transistor

IFCM inner field compensation method

IO integrated optics

MIC metal-insulator-cover impedance

QCM quartz crystal microbalance 
QELS quasielastic light scattering

RET resonance energy transfer

SAR scanning angle reflectometry

SAW surface acoustic wave

SCI semiconductor impedance

SPR surface plasmon resonance

TIRFM total internal reflexion fluorescence microscopy

TIRS total internal reflexion spectroscopy 